\title{
Systematic revision of dactylogyridean parasites (Monogenea) from cichlid fishes in Africa, the Levant and Madagascar
}

\author{
Antoine PARISELLE \\ Institut de Recherche pour le Développement, \\ Laboratoire d'écologie et de systématique, Centre de Belair, \\ BP 1386, 18524 Dakar (Sénégal) \\ antoine.pariselle@ird.fr
}

Louis EUZET

Station méditerranéenne de l'Environnement littoral, Université Montpellier II,

1 Quai de la Daurade, F-34200 Sète (France)

euzet@univ-montp2.fr

KEY WORDS

Monogenea,

Dactylogyridea,

Urogyridae,

Ancyrocephalidae,

Cichlidae,

Africa,

Levant,

Madagascar.

MOTS CLÉS

Monogenea,

Dactylogyridea,

Urogyridae,

Ancyrocephalidae,

Cichlidae,

Afrique,

Proche-Orient,

Madagascar.
Pariselle A. \& Euzet L. 2009. - Systematic revision of dactylogyridean parasites (Monogenea) from cichlid fishes in Africa, the Levant and Madagascar. Zoosystema 31 (4): 849-898.

\section{ABSTRACT}

Systematic revision is made of the six dactylogyridean genera (Monogenea) described on African, Levantine and Malagasy cichlid fishes: Urogyrus Bilong Bilong, Birgi \& Euzet, 1994 (including one species); Enterogyrus Paperna, 1963 (including eight species); Insulacleidus Rakotofiringa \& Euzet, 1983 (including three species); Onchobdella Paperna, 1968 (including eight species); Scutogyrus Pariselle \& Euzet, 1995 (including six species) and Cichlidogyrus Paperna, 1960 (including 71 species). Validities of species are discussed. Diagnoses and illustrated determination keys are given for all taxa.

\section{RÉSUMÉ}

Révision systématique des Monogènes Dactylogyridea parasites de poissons Cichlidae en Afrique, au Proche-Orient et à Madagascar.

Les auteurs font la révision systématique des six genres de Monogènes Dactylogyridea connus pour être parasites des poissons Cichlidae en Afrique, au Proche-Orient et à Madagascar: Urogyrus Bilong Bilong, Birgi \& Euzet, 1994 (comprenant une espèce); Enterogyrus Paperna, 1963 (comprenant huit espèces); Insulacleidus Rakotofiringa \& Euzet, 1983 (comprenant trois espèces); Onchobdella Paperna, 1968 (comprenant huit espèces); Scutogyrus Pariselle \& Euzet, 1995 (comprenant six espèces) et Cichlidogyrus Paperna, 1960 (comprenant 71 espèces). La validité de toutes les espèces est discutée. Les diagnoses et des clés d'identification illustrées sont données pour tous les taxons. 


\section{INTRODUCTION}

Today six genera, belonging to Dactylogyridea Bychowsky, 1937, have been proposed on African, Levantine and Malagasy cichlids (due to the specificity of Monogenea, the description of a new Quadriacanthus species [genus originally described from Siluriformes] from a tilapiine host by Paperna [1973] - i.e. Q. tilapiae from Oreochromis esculentus (Graham, 1928) - in the Victoria Lake is indubitably the result of a sampling error [mix of samples or contamination]. Oreochromogyrus mymensinghi described from Bangladesh by Ferdousi \& Chandra [2002] on introduced Oreochromis niloticus, has to be considerer as nomen inquirenda because the description of this new genus and new species is based on the misidentification of Cichlidogyrus larvae): one Urogyridae Bilong Bilong, Birgi \& Euzet, 1994 (Urogyrus Bilong Bilong, Birgi \& Euzet, 1994) and five Ancyrocephalidae Bychowsky, 1937 (Enterogyrus Paperna, 1963; Insulacleidus Rakotofiringa \& Euzet, 1983; Onchobdella Paperna, 1968; Scutogyrus Pariselle \& Euzet, 1995 and Cichlidogyrus Paperna, 1960). The large number of species described (especially in Cichlidogyrus), the lack of revision (except Price 1967; Paperna 1979 or Pariselle 1996) or of determination keys, and newly acquired genetic data (see Pouyaud et al. 2006), lead us to undertake a systematic revision of dactylogyridean parasites from cichlid fishes of these regions.

\section{MATERIAL AND METHODS}

Terminology used is that of Pariselle \& Euzet (1995c): uncinulus instead of hooklets; for large hooks, as "gripus" has not been accepted by parasitologists, we will use "anchor" instead of "hamulus", because this term is close to the Latin anchora, and describes well the working of these haptoral sclerites (see Lim et al. 2001).

Host species validities and spellings are given according to FishBase (Froese \& Pauly 2009): www. fishbase.net.

\section{ABBREVIATIONS}

BMNH The Natural History Museum, London;
CAS Institute of Parasitology, Czechoslovak Academy of Sciences, České Budějovice, Czech Republic;

MNHN Muséum national d'Histoire naturelle, Paris; MRAC Musée royal de l'Afrique centrale, Tervuren.

\section{SYSTEMATICS}

\author{
Family UROGYRIDAE \\ Bilong Bilong, Birgi \& Euzet, 1994 \\ Genus Urogyrus \\ Bilong Bilong, Birgi \& Euzet, 1994
}

TYPE AND ONLY SPECIES. — Urogyrus cichlidarum Bilong Bilong, Birgi \& Euzet, 1994, by original designation.

DiagNOSIs. - Urogyridae. Four pairs of cephalic glands. Two pairs of ocellae with lenses. Intestinal caeca without diverticula, joined posteriorly. One pair of ventral anchors; right anchor abortive. One transverse bar (ventral). Fourteen ventral uncinuli. Very large testis, median, inter-caecal. Vas deferens encircling left intestinal caeca. Seminal vesicle present. One prostatic reservoir. Male copulatory complex with penis and accessory piece in two parts forming pincer shape. Ovary on the anterior right side of testis. Straight vagina with dextrolateral opening. Parasite in the urinary bladder of African cichlids.

\section{Urogyrus cichlidarum \\ Bilong Bilong, Birgi \& Euzet, 1994}

Urogyrus cichlidarum Bilong Bilong, Birgi \& Euzet, 1994: 562-564, figs 1-3.

Type MATeRIAL. - Holotype: MNHN 114 HF Tk11. Paratype: MNHN 115 HF Tk12.

TYPE HOST. - Benitochromis batesii (Boulenger, 1901) (first species cited by Bilong Bilong et al. [1994]).

Type locality. - Mbila, Nyong Basin, Cameroon.

Additional hosts. - Parananochromis caudifasciatus (Boulenger, 1913); Pungu maclareni (Trewavas, 1962); Stomatepia pindu Trewavas, Green \& Corbet, 1972; and Tilapia sp.

Site. - Urinary bladder.

Additional localities. - Batouri, Kadei Basin, Cameroon on Parananochromis caudifasciatus; Barombi M'Bo Lake, Cameroon on Pungu maclareni and Stomatepia pindu; Sangmelima, Lobo River, Cameroon on Tilapia sp. 
Family ANCYROCEPHALIDAE Bychowsky, 1937

Genus Enterogyrus Paperna, 1963

TYPE SPECIES. - Enterogyrus cichlidarum Paperna, 1963, by original designation.

OTHER SPECIES INCLUDED. - Enterogyrus amieti Bilong Bilong, Euzet \& Birgi, 1996; E. barombiensis Bilong Bilong, Birgi \& Euzet, 1991; E. coronatus Pariselle, Lambert \& Euzet, 1991; E. crassus Bilong Bilong, Birgi \& Euzet, 1996; E. foratus Pariselle, Lambert \& Euzet, 1991; E. malmbergi Bilong Bilong, 1988; E. melenensis Bilong Bilong, Birgi \& Lambert, 1989.

Diagnosis. - Ancyrocephalidae. Pear-shaped body enlarged posteriorly; thick tegument transversely striated (except on haptor). Four ocellae, members of anterior pair wider apart and smaller than those of posterior, anterior with or without lenses. Pharynx muscular; short oesophagus followed by two lateral intestinal caeca without diverticula, joined posteriorly. Haptor cup-shaped, opening ventrally or in two parts: one bulbous and one peduncular shaped. Two unequal pairs of anchors. One ventral transverse bar, straight or V-shaped. Fourteen equal uncinuli, each with points orientated ventrally (haptor cup-shaped) or anteriorly (haptor bulbous and peduncular shaped). Testis at level of junction of intestinal caeca. Vas deferens encircling left intestinal caeca, widening to form a seminal vesicle, continuing as narrow duct to base of penis. Sub-median tubular and spirally coiled (clockwise) penis, enlarged at base; accessory piece absent. Ovary median pre-testicular, ovoid, sometimes U-shaped. Vagina not observed. Stomach parasite of Levantine and African cichlid fishes.

\section{REMARKS}

Two species are not considered in this study because they were described from the stomach of Etroplus suratensis (Block, 1790) in Asia. These species (Enterogyrus globodiscus (Kulkarni, 1969) and E. papernai Gussev \& Fernando, 1973) are characterised by having two transverse bars (vs. only one for African and Levantine species), according to Paperna (1979) we think that this difference will be sufficient to justify splitting Enterogyrus into two genera.

\section{Enterogyrus cichlidarum Paperna, 1963}

Enterogyrus cichlidarum Paperna, 1963: 183-186, figs 1-3. - Bilong Bilong et al. 1989: 104, figs 4, 5.

Enterogyrus niloticus Eid \& Negm, 1987: 79-84, figs 1-4. — Khidr 1990: 741, 742.
Type MATERIAL. - Holotype: Hebrew University, Department of Parasitology, no specified number.

TyPe HOST. - Of E. cichlidarum: Tilapia zillii (Gervais, 1848) (first species cited by Paperna [1963]).

Of E. niloticus: Oreochromis niloticus (Linnaeus, 1758).

Type locality. - Of E. cichlidarum: Rubin River, Israel.

Of E. niloticus: Barher Mouise, Nile River, Egypt.

ADDITIONAL HOSTS. - Sarotherodon galilaeus sanagaensis (Thys van den Audenaerde, 1966) and Tilapia nyongana Thys van den Audenaerde, 1971 (Bilong Bilong et al. 1996).

SITE. - Stomach.

ADDITIONAL LOCALITIES. - Jordan and coastal systems, Israel (Paperna 1979); South of Cameroon on O. niloticus (Bilong Bilong et al. 1989); Sakbayémé, Sanaga Basin, Cameroon on Sarotherodon galilaeus sanagaensis and So'o, Nyong Basin, Cameroon on Tilapia nyongana (Bilong Bilong et al. 1996).

\section{REMARKS}

The comparison by Khidr (1990) of Enterogyrus species specimens from Tilapia zillii and Oreochromis niloticus in Egypt revealed no differences and led this author to synonymise E. niloticus with E. cichlidarum.

\section{Enterogyrus amieti}

Bilong Bilong, Euzet \& Birgi, 1996

Enterogyrus amieti Bilong Bilong, Euzet \& Birgi, 1996: 38 , 39, figs $4,5$.

TyPe MATERIAL. - Holotype: MNHN 473 HF Tg72. Paratype: MRAC 37.362.

TYPE HOST. - Sarotherodon galilaeus sanagaensis (Thys van den Audenaerde, 1966).

Type locality. - Sakbayémé, Sanaga Basin, Cameroon.

SITE. - Stomach.

\section{Enterogyrus barombiensis}

Bilong Bilong, Birgi \& Euzet, 1991

Enterogyrus barombiensis Bilong Bilong, Birgi \& Euzet, 1991: 105, 106, figs 2, 3. 
TYPE MATERIAL. - Holotype: MNHN 111HF Tk7. Paratype: MNHN 112HF Tk8.

Type host. - Pungu maclareni (Trewavas, 1962).

Additional Hosts. - Stomatepia pindu Trewavas, 1972, Konia eisentrauti (Trewavas, 1962).

Type locality. - Barombi Mbo Crater Lake, Cameroon.

SiTE. - Stomach.

\section{Enterogyrus coronatus}

Pariselle, Lambert \& Euzet, 1991

Enterogyrus coronatus Pariselle, Lambert \& Euzet, 1991 : 213-216, figs $1 \mathrm{D}_{2}, 4,5 \mathrm{~A}$.

Type Material. - Holotype: MNHN 89 HF Tk1.

Paratypes: MNHN 89 HF Tk2; BMNH 1990.12.7.2.

TYPe HOST. - Tilapia guineensis (Bleeker, 1862).

Type Locality. - Layo station, Ebrié Lagoon, Côte d'Ivoire.

SITE. - Stomach.

\section{Enterogyrus crassus}

Bilong Bilong, Euzet \& Birgi, 1996

Enterogyrus crassus Bilong Bilong, Euzet \& Birgi, 1996: 38 , figs 2,3 .

TYPE MATERIAL. - Holotype: MNHN 472 HF Tg72. Paratype: MRAC 37.363.

TYPE HOST. - Tilapia nyongana Thys van den Audenaerde, 1971.

Type locality. — So'o, Nyong Basin, Cameroon.

SITE. - Stomach.

\section{Enterogyrus foratus}

Pariselle, Lambert \& Euzet, 1991

Enterogyrus foratus Pariselle, Lambert \& Euzet, 1991 : 212, 213, figs $1 \mathrm{~A}, 1 \mathrm{D}_{1}, 2,5 \mathrm{~B}$.

TYPE MATERIAL. - Holotype: MNHN 90 HF Tk3. Paratypes: MNHN 90 HF Tk4; BMNH 1990.12.7.1.

TYPE HOST. - Sarotherodon melanotheron heudelotii (Duméril, 1859).
Type Locality. - Mouth of the Casamance River, Senegal.

ADDITIONAL HOST. - Sarotherodon melanotheron melanotheron Rüppel, 1852.

SITE. — Stomach.

AdDitional Locality. - Layo station, Ebrié Lagoon, Côte d'Ivoire.

Enterogyrus malmbergi Bilong Bilong, 1988

Enterogyrus malmbergi Bilong Bilong, 1988: 52, 53, figs $1,2$.

Type Material. - No type material mentioned in the original description.

TYPE HOST. - Oreochromis niloticus (Linnaeus, 1758).

Type locality. - Edebda, Sanaga River, Cameroon.

SiTE. - Stomach.

\section{Enterogyrus melenensis}

Bilong Bilong, Birgi \& Lambert, 1989

Enterogyrus melenensis Bilong Bilong, Birgi \& Lambert, 1989: 101-104, figs 2, 3.

Type Material. - Holotype: MRAC 37.226.

Paratype: MRAC 37.227.

Type Host. - Hemichromis fasciatus Peters, 1857.

TyPe locality. — Melen, Yaoundé, Cameroon.

Site. - Stomach.

Additional localities. - Nyong, Sanaga and Lobé Basins, Cameroun.

\section{"Enterogyrus hemihaplochromii"}

"Enterogyrus hemihaplochromii" - Bender 1979.

Type Material. - No type material mentioned in the original description.

Host. - Pseudocrenilabrus multicolor Schoeller, 1903.

SITE. - Stomach.

LOCALITY. — East Africa. 


\section{REMARKS}

"Enterogyrus hemihaplochromii" was described in an unpublished thesis; this name is then a nomen nudum.

\section{Enterogyrus sp.}

Enterogyrus sp. - Cone et al. 1987: 312, 313, figs 1, 2,3 .

HosT. - Captive Pomacanthus paru (Bloch, 1787).

LOCALITY. - Caribbean Sea.

SITE. - Foregut.

\section{REMARKS}

According to Pariselle et al. (1991) this species, due to its morphology and anatomy, does not belong to Enterogyrus, but rather to Diplectanotrema Johnston \& Tiegs, 1922 or Pseudempleurosoma Yamaguti, 1965 which individuals are located in the pharynx of marine fishes.

\section{Genus Insulacleidus Rakotofiringa \& Euzet, 1983}

TYPE SPECIES. - Insulacleidus paratilapiae Rakotofiringa \& Euzet, 1983, by original designation.

OTHER SPECIES INCLUDED. - Insulacleidus ptychochromidis Rakotofiringa \& Euzet, 1983 and I. paretropli Rakotofiringa \& Euzet, 1983.

DiagnOSIS. - Ancyrocephalidae. Three pairs of cephalic glands. Two pairs of ocellae. Intestinal caeca without diverticula, joined posteriorly. Two pairs of anchor, one dorsal and one ventral. Two transverse bars, one dorsal one ventral. Fourteen uncinuli. Testis median posterior to the body. Vas deferens encircling left intestinal caeca. Seminal vesicle present. One prostatic reservoir. Male copulatory complex with penis and accessory piece. Pre-testicular median ovary. Vagina with lateral dextral opening. Seminal receptacle present. Gill parasite on Malagasy cichlid fishes.

\section{Insulacleidus paratilapiae}

Rakotofiringa \& Euzet, 1983

Insulacleidus paratilapiae Rakotofiringa \& Euzet, 1983: 107-110, figs 1-4.

TYPE MATERIAL. - No type material mentioned in the original description.
TYPE HOST. — Paratilapia polleni Bleeker, 1868.

TyPe LOCALITY. - No type locality mentionned in the original description.

SITE. — Gills.

Additional localities. - Near Majunga, West of Madagascar; Katsepy and Ampizoroa Lakes, Madagascar; Périmet and Ivoloina aquaculture stations, Tamatave Province, Madagascar.

\section{Insulacleidus paretropli}

Rakotofiringa \& Euzet, 1983

Insulacleidus paretropli Rakotofiringa \& Euzet, 1983: 111,112 , figs 8-10.

TYPE MATERIAL. - No type material mentioned in the original description.

TYPE HOST. - Paretroplus polyactis Bleeker, 1878.

TyPe LOCAlity. - Ambila, Antananarivo, Madagascar.

SITE. — Gills.

\section{Insulacleidus ptychochromidis}

Rakotofiringa \& Euzet, 1983

Insulacleidus ptychochromidis Rakotofiringa \& Euzet, 1983: 110, 111, figs 5-7.

TYPE MATERIAL. - No type material mentioned in the original description.

TYPe HOST. - Ptychochromis oligacanthus (Bleeker, 1868).

TyPe LOCAlity. - Ambila, Lemaitro, Tamatave Province, Madagascar.

SITE. — Gills.

\section{Genus Onchobdella Paperna, 1968}

TYPe SPeCIES. - Onchobdella voltensis Paperna, 1968, by original designation.

OTHER SPECIES INCLUDED. - Onchobdella aframae Paperna, 1968; O. bopeleti Bilong Bilong \& Euzet, 1995; O. krachii Paperna, 1968; O. melissa Pariselle \& Euzet, 
1995; O. pterigyalis Paperna, 1968; O. silverai Pariselle \& Euzet, 1995; O. spirocirra Paperna, 1968.

DiAgnosis (according to Pariselle \& Euzet [1995a]). Ancyrocephalidae. Three pairs of cephalic glands. Two posterior ocellae with lenses, two small anterior ocellae. Intestinal caeca without diverticula, joined posteriorly. Two pairs of anchors: one large dorsal, one smaller ventral. Three transverse bars: one dorsal, two ventral well separated. Fourteen uncinuli of same length. Testis posterior inter-caecal. Vas deferens encircling left intestinal caeca. Seminal vesicle present. One prostatic reservoir. Male copulatory complex with penis and accessory piece. Median pre-testicular ovary. Vaginal opening lateral, dextral, sclerotised. Gill parasites on African cichlid fishes.

\section{Onchobdella voltensis Paperna, 1968}

Onchobdella voltensis Paperna, 1968: 201, fig. 1.

TyPe MATERIAL. - Holotype: BMNH 1968.9.4.5. Paratype: BMNH 1968.9.4.6.

Type HOST. - Hemichromis fasciatus Peters, 1857.

Type LOCALITY. - Afram sector, Volta Lake, Ghana.

Additional HOST. - Hemichromis bimaculatus Gill, 1862.

SITE. — Gills.

Additional localities. - Kete Krachi, Volta Lake and Adutor Lagoon Volta River Delta, Ghana on $H$. bimaculatus; Benin and Cameroon on Hemichromis fasciatus (Dossou \& Birgi, 1984); Senegal, Gambia, Mali and Côte d'Ivoire on Hemichromis fasciatus (Pariselle \& Euzet 2004).

\section{Onchobdella aframae Paperna, 1968}

Onchobdella aframae Paperna, 1968: 205: fig. 4f-h.

TyPe Material. — Holotype: BMNH 1968.9.4.3.

Type Host. - Hemichromis fasciatus Peters, 1857.

TyPe LOCALITY. - Afram sector, Volta Lake, Ghana.

SITE. — Gills.

Additional localities. - Benin and Cameroon (Dossou \& Birgi 1984); Senegal, Gambia, Mali and Côte d'Ivoire (Pariselle \& Euzet 2004).

\section{REMARKS}

Dossou (1973), in his $\mathrm{PhD}$ Thesis on the fish parasites from South Benin, re-described $O$. aframae and indicated a new host: Pelmatochromis caudifasciatus Boulenger, 1913 (the species determination of this new host remains uncertain; in fact Parananochromis caudifasciatus (Boulenger, 1913) is limited to Southern Cameroon, the Northern part of the Congo and the Rio Muni; this distribution excludes the Benin rivers [see Froese \& Pauly 2009]).

\section{Onchobdella bopeleti \\ Bilong Bilong \& Euzet, 1995}

Onchobdella bopeleti Bilong Bilong \& Euzet, 1995: 254-256, figs 2-4.

TYPE MATERIAL. - Holotype: MNHN 471 HF Tg71. Paratypes: MNHN 471 HF Tg71; BMNH 1994.5.5.4; MRAC 37.367 and 37.368.

Type host. - Hemichromis fasciatus Peters, 1857.

TYPE LOCALITY. - No type locality mentionned in the original description.

SITE. — Gills.

Additional localities. - Kribi, Ejambwé River, Cameroon; Bamako, Niger River, Mali; Senegal, Gambia, Mali and Côte d'Ivoire (Pariselle \& Euzet 2004).

\section{Onchobdella krachii Paperna, 1968}

Onchobdella krachii Paperna, 1968: 205, figs 3d, 4c-e.

TYPe MATERIAL. - BMNH 1968.9.4.4; MRAC MT 35.574 (Paperna 1979).

TYPe HOST. - Chromidotilapia guentheri (Sauvage, 1882).

Type LOCAlity. - Kpandu and Kete Krachi, Volta Lake, Ghana.

SiTE. - Gills.

\section{Onchobdella melissa Pariselle \& Euzet, 1995}

Onchobdella melissa Pariselle \& Euzet, 1995a: 206, fig. 4.

TYPe MATERIAL. - Holotype: MNHN 466 HF Tg65. Paratypes: MNHN 467 HF Tg66; BMNH 1994 5.5.2; 
MRAC $37.365 \mathrm{~A}$ and $37.366 \mathrm{~B}$.

TyPe HOST. - Pelmatochromis buettikoferi (Steindachner, 1894).

Type Locality. — Koleya, Konkouré River, Guinea.

SITE. — Gills.

\section{Onchobdella pterigyalis Paperna, 1968}

Onchobdella pterigyalis Paperna, 1968: 203-205, figs $3 \mathrm{c}, \mathrm{e}, 4 \mathrm{a}, \mathrm{b}$.

Type Material. - Holotype: BMNH 1968.9.4.1.

Type HOST. - Hemichromis bimaculatus Gill, 1862.

Type locality. - Nunguia dam, East of Accra, Ghana.

SITE. - Fins.

AdDitional Site. — Gills.

ADDiTIONAL LOCALITY. - Mamahuma stream, East of Accra, Ghana

REMARKS

Dossou (1973), in his $\mathrm{PhD}$ thesis on the fish parasites from South Benin, re-described O. pterigyalis (as O. pterygialis) and indicated a new host: Pelmatochromis caudifasciatus Boulenger, 1913 (the species determination of this new host remains uncertain; in fact, it has been synonymised with Parananochromis caudifasciatus (Boulenger, 1913) which is limited to Southern Cameroon, the Northern part of the Congo and the Rio Muni; this distribution excludes the Benin Rivers).

\section{Onchobdella silverai Pariselle \& Euzet, 1995}

Onchobdella silverai Pariselle \& Euzet, 1995a: 206-208, fig. 5.

Type Material. - Holotype: MNHN 468 HF Tg67. Paratypes: MNHN 467 HF Tg66; BMNH 1994 5.5.3; MRAC $37.365 \mathrm{~B}$ and $37.366 \mathrm{~A}$.

TYPE HOST. - Pelmatochromis buettikoferi (Steindachner, 1894).

TyPe LOCALITY. — Koleya, Konkouré River, Guinea.

SITE. — Gills.
Onchobdella spirocirra Paperna, 1968

Onchobdella spirocirra Paperna, 1968: 201-203, figs 2, 3a.

Type MATERIAL. - Holotype: BMNH 1968.9.4.2.

TYPe HOST. - Hemichromis bimaculatus Gill, 1862.

TYPE LOCALITY. - No type locality mentionned in the original description.

SITE. — Gills.

AdDitional locality. - Afram sector, Volta Lake, Ghana.

Genus Scutogyrus Pariselle \& Euzet, 1995

TyPe SPECIES. - Scutogyrus minus (Dossou, 1982), by subsequent designation (Pariselle \& Euzet 1995b).

OTHER SPECIES INCLUDED. - Scutogyrus bailloni Pariselle \& Euzet, 1995; S. chikhii Pariselle \& Euzet, 1995; S. ecoutini Pariselle \& Euzet, 1995; S. gravivaginus (Paperna \& Thurston, 1969); S. longicornis (Paperna \& Thurston, 1969).

DiagNOSIS. - Ancyrocephalidae. Three pairs of cephalic glands. Two posterior ocellae with lenses. Two small anterior ocellae, sometimes absent. Intestinal caeca without diverticula, joined posteriorly. Two pairs of anchors, one dorsal and one ventral. Dorsal transverse bar highly arched, enlarged laterally, winged, having in its median portion two very long auricles hollow at their bases. Ventral transverse bar arched, rigid, supporting one large, thin oval plate marked by fan-shaped median thickenings. Fourteen uncinuli. Testis median, posterior to the body. Vas deferens dextral, not encircling intestinal caeca. Seminal vesicle present. One prostatic reservoir. Male copulatory complex with penis and accessory piece. Median pre-testicular ovary. Vaginal opening sub-lateral, dextral; vagina tubular, sclerotised. Seminal receptacle present. Gill parasites of African cichlid fishes.

\section{Scutogyrus minus (Dossou, 1982)}

Cichlidogyrus longicornis minus Dossou, 1982: 309-312, figs 19-23.

Cichlidogyrus minus - Pariselle \& Euzet 1995b: 158-161, figs $2,3$.

Scutogyrus minus - Pariselle \& Euzet 1995b.

Type MATERIAL. - Holotype: MNHN Tj141. 
Paratype: MNHN Tj142.

OTHER MATERIAL. - MNHN 460 HF Tg55; MNHN 460 HF Tg56 and Tg57; BMNH 1994.4.7.1; MRAC 37.357 (Pariselle \& Euzet 1995b).

TYPE HOST. - Sarotherodon melanotheron Rüppel, 1852.

Type LOCAlity. - Ouémé and Couffo Rivers, Benin.

SITE. — Gills.

Additional localities. - Guinea and Côte d'Ivoire (Pariselle \& Euzet 1995b).

\section{REMARKS}

Dossou (1982) suggested that Cichlidogyrus longicornis minus could be raised to species level. Douëllou (1993) indicated that this sub-species is uncertain and had to be invalidated. Pariselle \& Euzet (1995b) did not agree with Douëllou.

\section{Scutogyrus longicornis \\ (Paperna \& Thurston, 1969)}

Cichlidogyrus longicornis longicornis Paperna \& Thurston, 1969: 20-22, fig. 3a, b, d.

Cichlidogyrus longicornis - Douëllou 1993: 170-172, fig. $7_{1}, 7_{2}$.

Scutogyrus longicornis - Pariselle \& Euzet 1995b: 161163 , figs $4,5$.

Actinocleidus muelleri Ferdousi \& Chandra, 2002: 56, 57, fig. 4.

TYPE MATERIAL. - Holotype from Sarotherodon galilaeus: MRAC M.T. 35.931 (Paperna 1979).

Paratypes: private collection of Dr Paperna.

OTHER MATERIAL. - MNHN 128 HF (Douëllou 1993); MNHN 461 HF Tg58 and Tg59; BMNH 1994.4.7.2; MRAC 37.358 (Pariselle \& Euzet 1995b).

TYPE HOST. - Sarotherodon galilaeus (Linnaeus, 1758).

TyPe LOCAlity. — Lakes Georges and Albert, Uganda.

ADDITIONAL HOSTS. - Oreochromis niloticus (Linnaeus, 1758) and Tilapia zillii Gervais, 1848 (Paperna 1979) (for T. zillii: as species belonging to Scutogyrus are only found on mouthbreeder cichlid fishes, it is probably the result of a sampling error [mix of samples or contamination] or of host identification); Oreochromis mortimeri (Trewavas, 1966) (Douëllou 1993); O. mossambicus (Ferdousi \& Chandra 2002).

SITE. - Gills.

Additional LOCAlities. - Kete Kratchi, Volta Lake, Ghana (Paperna 1969); Cairo, Nile River, Egypt on O. niloticus (Ergens 1981); Kariba Lake, Zimbabwe on O. mortimeri (Douëllou 1993). Senegal and Côte d'Ivoire on O. niloticus (Pariselle \& Euzet 1995b). Bangladesh in BAU and BFRI fish farm ponds on introduced O. niloticus and O. mossambicus (Ferdousi \& Chandra 2002).

\section{REMARKS}

Dossou (1982) suggested that $C$. longicornis longicornis could be raised to species level, this was done by Douëllou (1993).

\section{Scutogyrus gravivaginus}

(Paperna \& Thurston, 1969)

Cichlidogyrus longicornis gravivaginus Paperna \& Thurston, 1969: 20-23, fig. 3c, e-g.

Cichlidogyrus gravivaginus - Douëllou 1993: 172, 173, fig. $7_{3}$.

Scutogyrus gravivaginus - Pariselle \& Euzet 1995b: 163165 , figs $6,7$.

Type Material. - Holotype: MRAC M.T. 35.932 (Paperna 1979).

Paratypes: private collection of Paperna.

OTHER MATERIAL. - MNHN 129 HF (Douëllou 1993).

TyPe HOST. - Oreochromis leucostictus (Trewavas, 1933).

TyPE LOCALITY. - Jinja and Albert Lake, Uganda.

ADDITIONAL HOSTS. - Oreochromis variabilis (Boulenger, 1906) (Paperna 1979); Oreochromis mortimeri (Trewavas, 1966) (Douëllou 1993).

SITE. - Gills.

Additional locality. - Kariba Lake, Zimbabwe on Oreochromis mortimeri (Trewavas, 1966) (Douëllou 1993).

\section{REMARKS}

Dossou (1982) suggested that $C$. longicornis gravivaginus could be raised to species level, this was done by Douëllou (1993). 


\section{Scutogyrus bailloni Pariselle \& Euzet, 1995}

Scutogyrus bailloni Pariselle \& Euzet, 1995b: 165, 166, figs $8,9$.

TyPe MATERIAL. - Holotype: MNHN 462 HF Tg60. Paratypes: MNHN 462 HF Tg61; BMNH 1994.4.7.3; MRAC 37.359.

TYPE HOST. - Sarotherodon galilaeus (Linnaeus, 1758).

Type locality. - W National Park, Mékrou River, Niger.

SITE. — Gills.

\section{Scutogyrus ecoutini Pariselle \& Euzet, 1995}

Scutogyrus ecoutini Pariselle \& Euzet, 1995b: 167, 168, figs 10,11 .

TyPe MATERIAL. - Holotype: MNHN 463 HF Tg62. Paratypes: MNHN 463 HF Tg63; BMNH 1994.4.7.4; MRAC 37.360.

TYPE HOST. - Sarotherodon occidentalis (Daget, 1962).

TyPE LOCALITY. — La Ramié, Bourouma River, Guinea.

SITE. — Gills.

Scutogyrus chikhii Pariselle \& Euzet, 1995

Scutogyrus chikhii Pariselle \& Euzet, 1995b: 171, 172, figs 12,13 .

Type MATERIAL. - Holotype: MNHN 464 HF Tg64. Paratypes: BMNH 1994.4.7.5; MRAC 37.361.

TYPe HOST. - Oreochromis mossambicus (Peters, 1852).

TyPe LOCALITY. - Cayo Lake, Congo.

SITE. — Gills.

\section{Genus Cichlidogyrus Paperna, 1960}

TYPE SPECIES. - Cichlidogyrus arthracanthus Paperna, 1960 , by original designation.

OTHER SPECIES INCLUDED. - Cichlidogyrus acerbus Dossou, 1982; C. aegypticus Ergens, 1981; C. agnesi Pariselle \& Euzet, 1994; C. albareti Pariselle \& Euzet, 1998; C. amieti Birgi \& Euzet, 1983; C. amphoratus Pariselle \& Euzet, 1995; C. anthemocolpos Dossou, 1982;
C. arfi Pariselle \& Euzet, 1995; C. berradae Pariselle \& Euzet, 2003; C. berrebii Pariselle \& Euzet, 1994; C. bifurcatus Paperna, 1960; C. bilongi Pariselle \& Euzet, 1996; C. bonhommei Pariselle \& Euzet, 1998; C. bouvii Pariselle \& Euzet, 1997; C. bychowskii (Markevich, 1934); C. cirratus Paperna, 1964; C. cubitus Dossou, 1982; C. dageti Dossou \& Birgi, 1984; C. digitatus Dossou, 1982; C. dionchus Paperna, 1968; C. dossoui Douëllou, 1993; C. douellouae Pariselle, Bilong Bilong \& Euzet, 2003; C. ergensi Dossou, 1982; C. euzeti Dossou \& Birgi, 1984; C. falcifer Dossou \& Birgi, 1984; C. flexicolpos Pariselle \& Euzet, 1995; C. fontanai Pariselle \& Euzet, 1997; C. gallus Pariselle \& Euzet, 1995; C. giostrai Pariselle, Bilong Bilong \& Euzet, 2003; C. guirali Pariselle \& Euzet, 1997; C. halinus Paperna, 1969; C. halli (Price \& Kirk, 1967); C. haplochromii Paperna \& Thurston, 1969; C. hemi Pariselle \& Euzet, 1998; C. inconsultans Birgi \& Lambert, 1986; C. karibae Douëllou, 1993; C. kothiasi Pariselle \& Euzet, 1994; C. kouassii N'Douba, Thys van den Audenaerde \& Pariselle, 1997; C. lagoonaris Paperna, 1969; C. legendrei Pariselle \& Euzet, 2003; C. lemoallei Pariselle \& Euzet, 2003; C. levequei Pariselle \& Euzet, 1996; C. longicirrus Paperna, 1965; C. longipenis Paperna \& Thurston, 1969; C. louipaysani Pariselle \& Euzet, 1994; C. microscutus Pariselle \& Euzet, 1996; C. nandidae Birgi \& Lambert, 1986; C. njinei Pariselle, Bilong Bilong \& Euzet, 2003; C. nuniezi Pariselle \& Euzet, 1998; C. ornatus Pariselle \& Euzet, 1995; C. ouedraogoi Pariselle \& Euzet, 1996; C. paganoi Pariselle \& Euzet, 1997; C. papernastrema Price, Peebles \& Bamford, 1969; C. philander Douëllou, 1993; C. pouyaudi Pariselle \& Euzet, 1994; C. quaestio Douëllou, 1993; C. reversati Pariselle \& Euzet, 2003; C. rognoni Pariselle, Bilong Bilong \& Euzet, 2003; C. sanjeani Pariselle \& Euzet, 1997; C. sanseoi Pariselle \& Euzet, 2004; C. sclerosus Paperna \& Thurston, 1969; C. slembroucki Pariselle \& Euzet, 1998; C. testificatus Dossou, 1982; C. teugelsi Pariselle \& Euzet, 2004; C. thurstonae Ergens, 1981; C. tiberianus Paperna, 1960; C. tilapiae Paperna, 1960; C. vexus Pariselle \& Euzet, 1995; C. yanni Pariselle \& Euzet, 1996; C. zambezensis Douëllou, 1993.

Diagnosis (according to Pariselle \& Euzet [1997]). Ancyrocephalidae. Three pairs of cephalic glands. Two posterior ocellae with crystalline lenses. Two small inconsistent anterior ocellae. Intestinal caeca unbranched, joined posteriorly. Two pairs of anchors, one dorsal and one ventral. Two transverse bars, dorsal with two auricles, ventral V-shaped. Fourteen uncinuli. Median posterior testis. Vas deferens on right side, not encircling intestinal caecum. Seminal vesicle present. One prostatic reservoir. Male copulatory complex with penis and accessory piece. Median pre-testicular ovary. Sub-median vaginal dextral opening. Vagina sclerotised or not. Seminal receptacle present. Gill parasites of African Cichlidae, Nandidae and Cyprinodontidae. 


\section{Cichlidogyrus arthracanthus Paperna, 1960}

Cichlidogyrus arthracanthus Paperna, 1960: 3-5, figs 1, 2.

TYPE MATERIAL. - No type material mentioned in the original description.

Type HOST. - Tilapia zillii (Gervais, 1848).

TYPE LOCALITY. - Fish culture research station, Dor, Israel.

AdDitional HOSTS. - Tristramella simonis (Günther, 1864) and T. sacra (Günther, 1865); Tilapia guineensis (Bleeker, 1862) (Pariselle \& Euzet 1995c).

SITE. — Gills.

AdDiTIONAL LOCALITIES. - Western shore, Sea of Galilee, Israel. Ghana (Paperna 1965, 1979), Uganda (Paperna \& Thurston 1969), Egypt (Ergens 1981), Senegal, Guinea, Ivory Coast and Congo (Pariselle \& Euzet 1995c).

\section{Cichlidogyrus acerbus Dossou, 1982}

Cichlidogyrus acerbus Dossou, 1982: 314-316, figs 2628.

TYPE MATERIAL. - Holotype: MNHN Tj135.

Paratype: MNHN Tj136.

TYPE HOST. - Sarotherodon melanotheron melanotheron Rüppell, 1852.

TyPe LOCality. - Ouémé and Couffo, Benin.

ADDITIONAL HOSTS. - Sarotherodon melanotheron heudelotii (Duméril, 1859) (Pariselle 1995).

SITE. — Gills.

Additional LoCALities. - Ebrié lagoon, Bakré Lake and Samakro, Côte d'Ivoire on type host (Pariselle 1995). Kogon River, Koba, Konkouré River, Forecariah, Guinea; Saint Louis, Retba, Somone Lagoon, Ziguinchor, Senegal; Banjul, Gambia on S. m. heudelotii (Pariselle 1995).

\section{Cichlidogyrus aegypticus Ergens, 1981}

Cichlidogyrus aegypticus Ergens, 1981: 211, fig. 7.

TYPe Material. - Originally deposited at the CAS, with no mention of reference number.

Voucher specimen: MNHN 484 HF Tg96 and Tg97 (Pariselle \& Euzet 1996).
Type HOST. — Tilapia zillii (Gervais, 1848).

Type locality. - Cairo, Nile River, Egypt.

Additional Hosts. - Tilapia guineensis (Bleeker, 1862) (Pariselle \& Euzet 1995c), Tilapia dageti Thys van den Audenaerde, 1971, Tilapia louka Thys van den Audenaerde, 1969 and Tilapia walteri Thys van den Audenaerde, 1968 (Pariselle \& Euzet 1996).

SITE. — Gills.

Additional localities. - Senegal, Guinea, and Congo on T. guineensis (Pariselle \& Euzet 1995c); Côte d'Ivoire and Burkina Faso on T. zillii, Côte d'Ivoire on T. dageti; Guinea on T. louka and T. walteri (Pariselle \& Euzet 1996).

\section{REMARKS}

Dossou (1982) described this species in Benin, but confused the identification with C. gallus Pariselle \& Euzet, 1995 (see below under Cichlidogyrus gallus). The specimens of $C$. aegypticus from T. louka and T. walteri show a shape of the vagina slightly different from that described on T. zillii, which was not deemed sufficient for Pariselle \& Euzet (1996) to create a new species.

Cichlidogyrus agnesi Pariselle \& Euzet, 1995

Cichlidogyrus agnesi Pariselle \& Euzet, 1995c: 189, 190, fig. 2.

TYPe MATERIAL. - Holotype: MNHN 220 HF Tk22. Paratypes: MNHN 220 HF Tk23, BMNH 1993.5.14.7, MRAC 37.338.

TYPE HOST. - Tilapia guineensis (Bleeker, 1862).

Type Locality. - Tiassalé, Bandama River, Côte d'Ivoire.

SITE. - Gills.

Additional localities. - Senegal, Guinea and Congo on type host [Pariselle \& Euzet 1996].

\section{Cichlidogyrus albareti Pariselle \& Euzet, 1998}

Cichlidogyrus albareti Pariselle \& Euzet, 1998: 275, 276, fig. 2.

TYPE MATERIAL. - Holotype: MNHN 543 HF Tk73. Paratypes: MNHN 543 HF Tk74, BMNH 1997.1.30.1, 
MRAC 37.408.

Type HOST. - Tilapia brevimanus Boulenger, 1911.

Type Locality. - La Ramié, Bourouma River, Guinea.

SITE. — Gills.

Additional locality. - Ndyarendi, Kogon River, Guinea.

Cichlidogyrus amieti Birgi \& Euzet, 1983

Cichlidogyrus amieti Birgi \& Euzet, 1983: 101-104, figs 1-3.

TYPE MATERIAL. - No type material mentioned in the original description.

TYPE HOST. - Aphyosemion cameronense (Boulenger, 1903) (first species cited by Birgi \& Euzet [1983]) (Nothobranchiinae Radda \& Pürzl, 1981).

Type locality. - Kala, Zamacoe, Cameroon.

AdDitional Host. - Aphyosemion obscurum (Ahl, 1924), junior synonym of $A$. cameronense.

SITE. — Gills.

REMARKS

Authors indicated in the original description that the presence of a Cichlidogyrus species on an Aphyosemion species is probably the result of a lateral transfer from a cichlid fish.

\section{Cichlidogyrus amphoratus}

Pariselle \& Euzet, 1996

Cichlidogyrus amphoratus Pariselle \& Euzet, 1996: 115, figs $6,7$.

TYPe MATERIAL. - Holotype: MNHN 486 HF Tg100 Paratypes: MNHN 486 HFTg99, BMNH 1996.1.5.16, MRAC 37.384.

TYPe HOST. - Tilapia louka Thys van den Audenaerde, 1969.

TYPE LOCALITY. — Laramié, Bourouma River, Guinea.

SITE. — Gills.
Cichlidogyrus anthemocolpos Dossou, 1982

Cichlidogyrus anthemocolpos Dossou, 1982: 307-309, figs 16-18.

Type Material. - Holotype: MNHN Tj133.

Paratype: MNHN Tj134.

Type HOST. — Tilapia zillii (Gervais, 1848).

TyPe LOCALITY. — Ouémé, Benin.

SITE. — Gills.

Cichlidogyrus arfii Pariselle \& Euzet, 1995

Cichlidogyrus arfii Pariselle \& Euzet, 1995a: 204, 205, fig. 3.

Cichlidogyrus dionchus Paperna, 1968: 91, pl. 1, fig. 7.

Type MATERIAL. - Holotype: MNHN 470 HF Tg69. Paratypes: MNHN 470 HF Tg70, BMNH 1994.5.5.1, MRAC 37.364

TYPe HOST. — Pelmatochromis buettikoferi (Steindachner, 1894).

TyPe LOCAlity. — Koleya, Konkouré River, Guinea.

SITE. — Gills.

\section{REMARKS}

In the original description of $C$. dionchus, Paperna (1968) gave three different illustrations for copulatory organs, the figure 7 pl. 1 p. 91 (without references to host) resemble $C$. arfii, thus corresponding individuals have to be considered as $C$. arfi.

\section{Cichlidogyrus berradae Pariselle \& Euzet, 2003}

Cichlidogyrus berradae Pariselle \& Euzet, 2003: 196, fig. 3.

TYPE MATERIAL. - Holotype: MNHN 538 HF Tk63. Paratypes: MNHN 538 HF Tk64, BMNH 1997.1.29.1, MRAC 37 409, CAS M-385.

Type host. - Tilapia cabrae Boulenger, 1899.

Type LOCAliTY. - Cayo Lake, Cabinda.

AdDitional HOST. - Tilapia guineensis (Bleeker, 1862).

SITE. — Gills. 
Additional locality. - Loufoualéba Lake, Congo on T. guineensis.

\section{Cichlidogyrus berrebii Pariselle \& Euzet, 1994}

Cichlidogyrus berrebii Pariselle \& Euzet, 1994: 230, fig. 2.

TYPE MATERIAL. - Holotype and paratypes: MNHN 213 HF Tk14.

Paratypes: BMNH 1993.3.4.1; MRAC 37.330, 37.331 and 37.333 .

TYPE HOST. - Tylochromis jentinki (Steindachner, 1895)

TyPe LOCALITY. - Ebrié Lagoon, Côte d'Ivoire.

SITE. — Gills.

Additional localities. - Kogon River, Guinea and Sine Saloum Rivers, Senegal.

\section{REMARKS}

Pariselle \& Euzet (1994) noticed the unusual morphology of the dorsal transverse bar of Cichlidogyrus from Tylochromis Regan, 1920 hosts (auricles are continuous with convex face instead of attached on this face); pending new morphological or molecular data from these or other parasites from Tylochromis, they left these species in Cichlidogyrus.

\section{Cichlidogyrus bifurcatus Paperna, 1960}

Cichlidogyrus bifurcatus Paperna, 1960: 13, 14, fig. 9; 1979: 9, pl. 1, figs 1-6.

Type Material. - Paratype: MRAC M.T. 35.703 (Paperna, 1979).

TYPE HOST. - Haplochromis flaviijosephi (Lortet, 1883).

Type LOCALITY. — Sea of Galilee, Israel.

AdDitional HOSTS. - Juveniles of Oreochromis aureus (Steindachner, 1864); Haplochromis squamipinnis Regan, 1921, H. elegans Trewavas, 1933, H. aeneocolor Greenwood, 1973, H. limax Trewavas, 1933, H. sp. and Pseudocrenilabrus multicolor (Schoeller, 1903) (Paperna 1979).

SITE. — Gills.

\section{REMARKS}

Douëllou (1993) thought that C. bifurcatus was probably, at times, confused with $C$. haplochromii, we do not agree: $C$. bifurcatus is a valid species, as its description, illustrations and hosts never vary in Paperna publications (1960, 1979).

\section{Cichlidogyrus bilongi Pariselle \& Euzet, 1995}

Cichlidogyrus bilongi Pariselle \& Euzet, 1995c: 190, 191, fig. 3.

Type MATERIAL. - Holotype: MNHN 218 HF Tk18. Paratypes: MNHN 218 HF Tk19, BMNH 1993.5.14.8, MRAC 37.337.

TYPE HOST. - Tilapia guineensis (Bleeker, 1862).

TyPe LOCAliTY. - Tiassalé, Bandama River, Guinea.

SITE. - Gills.

AdDitional LOCALITY. - Layo research station, Ebrié Lagoon, Côte d'Ivoire. Senegal and Congo on type host (nobis).

\section{Cichlidogyrus bonhommei}

Pariselle \& Euzet, 1998

Cichlidogyrus bonhommei Pariselle \& Euzet, 1998: 279, 280, fig. 5.

TyPe MATERIAL. - Holotype: MNHN 545 HF Tk77. Paratypes: MNHN 545 HFTk78, BMNH 1997.1.30.2, MRAC 37.403.

Type HOST. - Tilapia buttikoferi (Hubrecht, 1881).

TyPE LOCALITY. — Katonga, Little Scarcies River, Sierra Leone.

SITE. — Gills.

Cichlidogyrus bouvii Pariselle \& Euzet, 1997

Cichlidogyrus bouvii Pariselle \& Euzet, 1997: 223, fig. 2.

Type Material. - Holotype: MNHN 530 HF Tk48. Paratypes: MNHN 530 HF Tk49, BMNH 1997.1.28.2, MRAC 37.395.

TYPE HOST. - Sarotherodon occidentalis (Daget, 1962). TYPE LOCALITY. — La Ramié, Bourouma River, Guinea. SiTE. - Gills.

Additional localities. - Kamouri, Batapon River, Guinea; Katonga, Little Scarcies River, Sierra Leone on type host. 


\section{Cichlidogyrus bychowskii (Markevich, 1934)}

Ancyrocephalus bychowskii Markevich, 1934.

Cichlidogyrus bychowskii - Paperna 1960: 14.

TYPe MATERial. - No type material mentioned in the original description.

TyPe HOST. - Hemichromis bimaculatus Gill, 1862.

TYPE LOCALITY. — From a fish reared in the Leningrad aquarium.

Additional hosts. - Hemichromis fasciatus Peters, 1857 and Sarotherodon galilaeus (Linnaeus, 1758) [Paperna 1968, 1969].

SITE. — Gills.

ADDiTIONAL LOCALITIES. - Ghana on type host (Paperna 1965); Ghana on type host, Hemichromis fasciatus Peters, 1857 and Sarotherodon galilaeus (Linnaeus, 1758) (Paperna 1968, 1969).

\section{Cichlidogyrus cirratus Paperna, 1964}

Cichlidogyrus cirratus Paperna, 1964: 4, 5, figs 10, 11.

Cichlidogyrus nematocirrus Paperna, 1969: 863, figs 54, 55; 1979: 13, pl. 3, figs 3-7.

TYPE MATERIAL. - Of C. cirratus: Holotype and paratypes in the helminthological collection of the Department of Parasitology Hebrew University, Hassad Medical School, Israel, no number mentioned.

Of C. nematocirrus: Holotype: MRAC M.T. 35.583 (Paperna 1979).

TyPe HOST. - Of C. cirratus: Sarotherodon galilaeus (Linnaeus, 1758).

Of C. nematocirrus: Oreochromis niloticus (Linnaeus, 1758) (Paperna 1979).

TyPE LOCALITY. - Of C. cirratus Tiberias Lake, Israel. Of C. nematocirrus: Man-made Volta Lake, Ghana.

AdDitional host. - Of C. cirratus: Oreochromis niloticus (Ergens 1981).

Of C. nematocirrus: Tilapia zillii (Gervais, 1848), Oreochromis variabilis (Boulenger, 1906) and Oreochromis esculentus (Graham, 1928) (Paperna 1979).

SITE. — Gills.

Additional localities. - Cairo, Nile River, Egypt (Ergens 1981) on O. niloticus. Senegal on type host (nobis).

\section{REMARKS}

Cichlidogyrus cirratus and C. nematocirrus have high morphological similarities and are both found on $O$. niloticus. The only difference between these two species is the length of the penis (320 vs. $145 \mu \mathrm{m}$ ) which is not easy to measure (very long, sinuous and thin penis), thus we consider that $C$. nematocirrus is a junior synonym of $C$. cirratus.

\section{Cichlidogyrus cubitus Dossou, 1982}

Cichlidogyrus cubitus Dossou, 1982: 306, 307, figs 1315.

Type MATERIAL. - Holotype: MNHN Tj114.

Paratype: MNHN Tj115.

Type HOST. - Tilapia zillii (Gervais, 1848).

TYPe LOCALITY. - Ouémé, Benin.

SITE. - Gills.

\section{Cichlidogyrus dageti Dossou \& Birgi, 1984}

Cichlidogyrus dageti Dossou \& Birgi, 1984: 106-108, figs 9-11.

TyPe MATERIAL. - Holotype: MNHN 208 TC Tj134.

TyPe HOST. - Hemichromis fasciatus Peters, 1857.

TYPE LOCALITY. - No type locality mentionned in the original description.

SITE. — Gills.

Additional locality. - Nyong Basin, Cameroon on type host.

\section{Cichlidogyrus digitatus Dossou, 1982}

Cichlidogyrus digitatus Dossou, 1982: 303-306, figs 1012.

Cichlidogyrus cf. brevicirrus - Paperna 1969: 864, figs 78, 79.

Cichlidogyrus halinus Paperna, 1969 (part): 860, 861, fig. 71 left drawing (individuals from Tilapia guineensis in the original description) (cf. Pariselle \& Euzet 1996: 117, 118, fig. 10). 
Type Material. $-C$. digitatus: Holotype: MNHN Tj116. Paratype: MNHN Tj117.

OTHER MATERIAL. - C. cf. brevicirrus: "paratype": MRAC M.T. 35.586 from Tilapia discolor (Günther, 1903) (Paperna 1979) (see Remarks under Cichlidogyrus dionchus chapter below).

TyPe HOST. - C. digitatus: Tilapia zillii (Gervais, 1848).

TYPe LOCALiTY. - Ouémé, Benin.

AdDitional HOSTs. - Tilapia guineensis (Bleeker, 1862) (Paperna 1969). Tilapia dageti Thys van den Audenaerde, 1971, T. guineensis (Bleeker, 1862) and T. louka Thys van den Audenaerde, 1969 (Pariselle \& Euzet 1996). Tilapia brevimanus Boulenger, 1911 (Pariselle \& Euzet 1998).

SITE. — Gills.

Additional localities. - Volta Lake, Ghana on Tilapia guineensis (original description of $C$. halinus in Paperna 1969). Senegal, Guinea, Côte d'Ivoire and Congo on Tilapia guineensis (Pariselle \& Euzet 1995c); Côte d'Ivoire, Guinea, Senegal, Mali and Gambia on Tilapia zillii, T. dageti, T. guineensis and T. louka (Pariselle \& Euzet1996); Guinea on T. brevimanus (Pariselle \& Euzet 1998).

\section{REMARKS}

In our opinion, measurements and figures given in the original description of $C$. cf. brevicirrus by Paperna (1969) are almost identical to those of $C$. digitatus Dossou, 1982, with which this species had to be synonymised, despite the opinion of Paperna (1979), who synonymised it with $C$. dionchus.

Pariselle \& Euzet 1996 synonymised a part of the individuals named $C$. halinus with $C$. digitatus (those found on Tilapia guineensis by Paperna [1969: fig 71 left drawing]).

\section{Cichlidogyrus dionchus Paperna, 1968}

Cichlidogyrus dionchus Paperna, 1968: 89, 90, pl. 1, figs 4, 5.

Cichlidogyrus brevicirrus Paperna \& Thurston, 1969: 17, 18. - Paperna 1979: 9, 10.

TYPE MATERIAL. - Holotype: MRAC M.T. 35.585 from type host (Paperna 1979).

Type HOST. - Of C. dionchus: Sarotherodon galilaeus (Linnaeus, 1758).

Of C. brevicirrus: Haplochromis guiarti (Pellegrin, 1904).
TYPE LOCALITY. - Of C. dionchus: Mamahuma stream, Accra, Ghana.

Of C. brevicirrus: Jinja, Kazi, Victoria Lake, Uganda.

AdDitional Hosts. - Of C. dionchus: Chromidotilapia guentheri (Sauvage, 1882) (Paperna 1979).

Of C. brevicirrus: Haplochromis longirostris (Hilgendorf, 1888), Haplochromis obesus (Boulenger, 1906), Haplochromis obliquidens (Hilgendorf, 1888), Haplochromis sp., Hoplotilapia retrodens (Hilgendorf, 1888) and Tilapia zillii (Gervais, 1848).

SITE. — Gills.

Additional localities. - Afram sector, Volta Lake and Adutor lower reaches of the Volta River, Ghana on H. fasciatus; Ghana on Chromidotilapia guentheri (Paperna 1979).

\section{REMARKS}

Paperna (1968) used for this species two spellings: $C$. dioncus and $C$. dionchus, as the first one was latter use by Paperna (1979) and other authors, we accept this spelling. Dossou \& Birgi (1984) indicated that a proportion of individuals named $C$. dionchus by Paperna (1968: fig. 6, p. 91) correspond to C. falcifer (individuals from Hemichromisfasciatus) and we agree with that. Dossou (1982) indicated that $C$. dionchus (on Sarotherodon galilaeus) is a valid species. Some hosts were added by Paperna (1979): Pseudocrenilabrus multicolor (Schoeller, 1903), Tilapia rendalli (Boulenger, 1897) and Haplochromis wingatii (Boulenger, 1902), but probably because of the presence of $C$. $\mathrm{cf}$. brevicirrus, which was synonymised by this author with $C$. dionchus, when we propose to synonymise C. cf. brevicirrus with $C$. digitatus. In the same way, Paperna (1979) deposits a paratype (MRAC M.T. 35.586, probably $C$. cf. brevicirrus) from Tilapia discolor (Günther, 1903) for C. dionchus, we consider this specimen as being a $C$. digitatus. In the original description by Paperna (1968) the only true C. dionchus was illustrated by the drawing 5 (pl. 1, p. 91) and by the figures of $C$. brevicirrus in its original description (Paperna \& Thurston 1969: 18), this latter species had been synonymised with $C$. dionchus by Paperna (1979); we agree with this conclusion.

Cichlidogyrus dossoui Douëllou, 1993

Cichlidogyrus dossoui Douëllou, 1993: 174, 175, fig. 9. 
Type Material. - Holotype: MNHN 139 HF.

TYPE HOST. - Tilapia rendalli (Boulenger, 1897).

Type LOCALity. - Kariba Lake, Zimbabwe.

ADDITIONAL HOSTS. - Oreochromis mortimeri (Trewavas, 1966) and Serranochromis macrocephalus (Boulenger, 1899).

SITE. — Gills.

\section{Cichlidogyrus douellouae}

Pariselle, Bilong Bilong \& Euzet, 2003

Cichlidogyrus douellouae Pariselle, Bilong Bilong \& Euzet, 2003: 203-205, fig. 3.

Type Material. - Holotype: MNHN 535 HF Tk58. Paratypes: MNHN 535 HF Tk59, BMNH 1997.2.3.1, MRAC 37.410.

TYPE HOST. - Sarotherodon galilaeus (Linnaeus, 1758).

TyPe locality. - W National Park, Mékrou River, Niger.

ADDITIONAL HOST. - Sarotherodon galilaeus sanagaensis (Thys van den Audenaerde, 1966).

SITE. — Gills.

Additional localities. — Bama, Kou River, Burkina Faso on type host, Edéa, Sanaga River, Cameroon on S. g. sanagaensis.

\section{Cichlidogyrus ergensi Dossou, 1982}

Cichlidogyrus ergensi Dossou, 1982: 301-303, figs 7-9.

Cichlidogyrus sp. — Ergens 1981: 212, 213.

Type MATERIAL. — Holotype: MNHN Tj113.

TYPe HOST. — Tilapia zillii (Gervais, 1848).

Type locality. - Ouémé, Benin.

AdDitional Host. - Tilapia guineensis (Bleeker, 1862) (Pariselle \& Euzet 1995c).

SITE. — Gills.

Additional localities. - Cairo, Nile River, Egypt (description of Cichlidogyrus sp. by Ergens [1981]); Senegal, Guinea, Côte d'Ivoire and Congo on Tilapia guineensis (Bleeker, 1862) (Pariselle \& Euzet 1995c).
Cichlidogyrus euzeti Dossou \& Birgi, 1984

Cichlidogyrus euzeti Dossou \& Birgi, 1984: 108, 109, figs 12-14.

Type MATERIAL. - Holotype: MNHN 209 TC Tj135.

Type HOST. - Hemichromis fasciatus Peters, 1857.

TyPe LOCAlity. - Benin and Cameroon.

SITE. — Gills.

AdDitional Locality. - Congo on type host (Pariselle \& Euzet 2004)

Cichlidogyrus falcifer Dossou \& Birgi, 1984

Cichlidogyrus falcifer Dossou \& Birgi, 1984: 104-106, figs 5-8.

Cichlidogyrus dionchus Paperna, 1968 (part): 89-91, pl. 1, fig. 6 (individuals coming from Hemichromis fasciatus) (cf. Dossou \& Birgi 1984: 104-106).

TyPe MATERIAL. - Holotype: MNHN 207 TC Tj133.

Type HOst. - Hemichromis fasciatus Peters, 1857.

Type LOCALITY. - Benin and Cameroon.

SITE. — Gills.

Additional localities. - Congo, Senegal, Mali, Gambia and Côte d'Ivoire on type host (Pariselle \& Euzet 2004).

\section{REMARKS}

Dossou \& Birgi (1984) indicated that C. falcifer corresponds to a proportion of specimens named C. dionchus by Paperna in 1968; we agree with that: among the three figures given by Paperna (1968: 91), we consider number 6 (pl. I) to be a $C$. falcifer and probably was found on $H$. fasciatus.

Cichlidogyrus flexicolpos Pariselle \& Euzet, 1995

Cichlidogyrus flexicolpos Pariselle \& Euzet, 1995c: 193, 194, fig. 5.

Type MATERial. - Holotype: MNHN 217 HF Tk16. Paratypes: MNHN 217 HF Tk17, BMNH 1993.5.14.9, MRAC 37.340. 
TYPe HOST. — Tilapia guineensis (Bleeker, 1862).

Type Locality. - Tiassalé, Bandama River, Côte d'Ivoire.

Additional host. - Tilapia dageti Thys van den Audenaerde, 1971 (Pariselle \& Euzet 1996).

SITE. — Gills.

Additional localities. — Layo research station, Ebrié Lagoon, Côte d'Ivoire; Congo River, Congo on type host. Senegal and Guinea on T. guineensis and T. dageti (Pariselle \& Euzet 1996).

\section{Cichlidogyrus fontanai Pariselle \& Euzet, 1997}

Cichlidogyrus fontanai Pariselle \& Euzet, 1997: 223, 224, fig. 3.

TyPe MATERIAL. - Holotype: MNHN 532 HF Tk53. Paratypes: MNHN 532 HF Tk 53, BMNH 1997.1.28.3, MRAC 37.396.

TYPE HOST. - Sarotherodon occidentalis (Daget, 1962).

Type LOCAlity. — La Ramié, Bourouma River, Guinea.

SITE. — Gills.

Additional locality. - Kamouri, Batapon River, Guinea on type host.

Cichlidogyrus gallus Pariselle \& Euzet, 1995

Cichlidogyrus gallus Pariselle \& Euzet, 1995c: 191-193, fig. 4.

Cichlidogyrus aegypticus - Dossou 1982: 296-299, figs 1-3 (cf. Pariselle \& Euzet 1995c: 191). Not C. aegypicus Ergens, 1981.

TYPE MATERIAL. - Holotype: MNHN 221 HF Tk24. Paratypes: MNHN 221 HFTk25, BMNH 1993.5.14.10, MRAC 37.336.

TYPE HOST. - Tilapia guineensis (Bleeker, 1862).

Type locality. - Tiassalé, Bandama River, Côte d'Ivoire.

Additional hosts. - Tilapia zillii (Gervais, 1848) (Dossou 1982). Tilapia walteri Thys van den Audenaerde, 1968 (Pariselle \& Euzet 1996).

SITE. — Gills.

AdDitional localities. — Layo research station, Ebrié Lagoon, Côte d'Ivoire. Ouémé River, Benin on T. zil- lii (Dossou 1982). Senegal, Guinea, and Congo on T. guineensis and T. walteri (Pariselle \& Euzet 1996).

\section{Cichlidogyrus giostrai}

Pariselle, Bilong Bilong \& Euzet, 2003

Cichlidogyrus giostrai Pariselle, Bilong Bilong \& Euzet, 2003: 205, 206, fig. 4.

Type Material. - Holotype: MNHN 529 HF Tk46. Paratypes: MNHN 529 HF Tk47, BMNH 1997.2.3.2, MRAC 37.406 .

TYPE HOST. - Sarotherodon caudomarginatus (Boulenger, 1916).

Type locality. - Tondon Road, Badi River, Guinea.

Site. - Gills.

\section{Cichlidogyrus guirali Pariselle \& Euzet, 1997}

Cichlidogyrus guirali Pariselle \& Euzet, 1997: 224-226, fig. 4.

Type Material. - Holotype: MNHN 533 HF Tk54. Paratypes: MNHN 533 HF Tk55, BMNH 1996.1.5.2225, MRAC 37.386.

TYPE HOST. - Sarotherodon occidentalis (Daget, 1962).

TyPE LOCALITY. — La Ramié, Bourouma River, Guinea.

SITE. — Gills.

AdDITIONAL LOCALITIES. - Kamouri, Batapon River, Guinea and Little Scarcies River, Sierra Leone on type host.

\section{Cichlidogyrus halinus Paperna, 1969}

Cichlidogyrus halinus Paperna, 1969: 860, 861, figs 70, 71 right drawing, 72 .

Cichlidogyrus erectus Dossou, 1982: 316-318, fig. 29-30 (cf. Pariselle \& Euzet 1996: 117).

Type MATERIAL. - Of $C$. halinus: Holotype: MRAC M.T. 35.928 (Paperna 1979).

Of C. erectus: Holotype: MNHN Tj 143.

TYPE HOST. - Of C. halinus: Sarotherodon melanotheron heudelotii (Duméril, 1861).

Of $C$. erectus: Sarotherodon melanotheron melanotheron Rüppell, 1852.

Type Locality. - Of $C$. halinus: Peshs lagoon, east of Accra, South Ghana.

Of $C$. erectus: Ouémé and Couffo, Benin. 
SITE. — Gills.

\section{Cichlidogyrus halli (Price \& Kirk, 1967)}

Cleidodiscus halli Price \& Kirk, 1967: 139-141, figs 1-12. - Paperna 1979: 10.

Cichlidogyrus halli typicus Paperna, 1979: 11, pl. 2, fig. 1. — Douëllou 1993: 160-164, fig. 3.

Cichlidogyrus tubicirrus magnus Paperna \& Thurston, 1969: 26, fig. 6a, e. - Paperna 1979: 10.

Cichlidogyrus magnus - Dossou 1982: 312-314, figs 24, 25. — Douëllou 1993: 160-164.

Cichlidogyrus halli victorianus Paperna, 1979: 11, 12, pl. 2, figs 2, 3 .

Type material. - Of $C$. halli: Holotype: Albany Museum, Grahamstown, Republic of South Africa. Paratypes: Helminthological Collection, United States National Museum, Washington, DC; MRAC, no numbers mentioned. Voucher specimen from type host: MNHN 126 HF (Douëllou 1993).

Of C. halli typicus: Paratype: M.T. 35.511 from Oreochromis niloticus (only type specimen mentioned by Paperna 1979).

Of C. tubicirrus magnus: "Types": private collection of Paperna, no number mentioned.

Of C. halli victorianus: Holotype: MRAC M.T. 35.941 from Oreochromis variabilis (Paperna 1979).

TYPe HOST. - Of C. halli and C. halli typicus: Oreochromis shiranus shiranus (Boulanger, 1897) (original host in Price \& Kirk 1967).

Of C. tubicirrus magnus: Oreochromis niloticus (Linnaeus, 1758)

Of C. magnus: Sarotherodon melanotheron Rüppel, 1852 (in Dossou 1982).

Of C. halli victorianus: Oreochromis variabilis (Boulenger, 1906).

Type locality. — Of C. halli and C. halli typicus: Fort Johnston, Upper Shire River, Malawi.

Of C. tubicirrus magnus: Albert and Georges Lakes, Uganda.

Of $C$. magnus: Ouémé and Couffo, Bénin.

Of C. halli victorianus: Victoria Lake, Entebbe and Jinja, Uganda.

AdDitional hosts. - C. halli: Oreochromis mortimeri (Trewavas, 1966) and Serranochromis macrocephalus (Boulenger, 1899) (Douëllou 1993). Sarotherodon occidentalis (Daget, 1962) (Pariselle \& Euzet 1997).

C. halli typicus: Oreochromis niloticus (Linnaeus, 1758), Sarotherodon galilaeus (Linnaeus, 1758), Oreochromis leucostictus (Trewavas, 1933), Oreochromis niloticus vulcani (Trewavas,
1933) and Oreochromis spilurus spilurus (Günther, 1894). C. halli victorianus: Oreochromis esculentus (Graham, 1928) (Paperna 1979).

C. tubicirrus magnus: Sarotherodon galilaeus (Linnaeus, 1758), Oreochromis leucostictus (Trewavas, 1933).

SITE. — Gills.

Additional localities. - Of C. halli: Kariba Lake, Zimbabwe on Oreochromis mortimeri and Serranochromis macrocephalus (Douëllou 1993). Guinea and Sierra Leone on Sarotherodon occidentalis (Pariselle \& Euzet 1997).

Of $C$. halli typicus: lakes Georges, Albert and Edward, Kajansi fish ponds, Uganda on O. niloticus; Lake Albert, Uganda and Volta Lake and lower Volta River, Ghana on S. galilaeus; lakes Georges and Albert; Uganda on O. leucostictus; Kajansi fish ponds, Uganda on introduced $O$. niloticus vulcani and $O$. spilurus spilurus.

\section{REMARKS}

Price \& Kirk (1967) synonymised Cichlidogyrus with Cleidodiscus Mueller, 1934; this was confirmed by Price (1967). Price et al. (1969) rehabilitated Cichlidogyrus with five species (C. arthracanthus, C. bifurcatus, C. bychowskii, C. longicirrus and C. tiberianus), but kept in Cleidodiscus (valid genus) two species (C. halli and C. tilapiae). Paperna (1979) move back to Cichlidogyrus the two Cleidodiscus species found on African cichlid fishes (C. halli and C. tilapiae); this was confirmed by Douëllou (1993).

Considering measurements and figures by Paperna (1979: 11, pl. 2 figs 2, 3) and after the examination of type material, we did not find significant differences between $C$. halli victorianus and $C$. halli. So, despite the opinion of Douëllou (1993), we consider that $C . h$. victorianus had to be synonymised with $C$. halli.

The determination by Ergens (1981) of Tilapia zillii (Gervais, 1848) as host for C. halli in Egypt remains for us doubtful, in fact, this species is only found on mouthbreeders Cichlidae (i.e. genera Oreochromis and Sarotherodon).

\section{Cichlidogyrus haplochromii \\ Paperna \& Thurston, 1969}

Cichlidogyrus haplochromii Paperna \& Thurston, 1969: 18-20, fig. 2. - Paperna 1979: 12, pl. 3, figs 1, 2. Douëllou 1993: 168-170, fig. 6.

Cichlidogyrus dionchus Paperna, 1968 (see Remarks below). — Paperna 1979: 9, 10, pl. 1, fig. 7. 
Cichlidogyrus tubicirrus minutus Paperna \& Thurston, 1969: 26-28, fig. 6b, f (individuals from haplochromiine hosts).

Cichlidogyrus tubicirrus - Paperna 1979: 14 (individuals from haplochromiine hosts).

Type Material. - "Types": private collection of Dr Paperna, no number mentioned.

Of C. tubicirrus: "Types": private collection of Paperna, no number mentioned (original description of $C$. tubicirrus minutus); "Holotype": MRAC M.T. 34.291 from Haplochromis schubotzi Boulenger, 1914 (Paperna 1979).

Other Material. - MNHN 134 HF (Douëllou 1993).

TYPE HOST. - Haplochromis guiarti (Pellegrin, 1904). Of C. tubicirrus: Haplochromis schubotzi (Paperna 1979).

Type locality. - Jinja, Uganda for C. haplochromii and C. tubicirrus.

AdDiTIONAL HOSTS. - C. haplochromii: Haplochromis macrognathus Regan, 1922, Haplochromis nubilus (Boulenger, 1906), Haplochromis obliquidens (Hilgendorf, 1888), Haplochromis wingatii (Boulenger, 1902), Haplochromis sp., Hoplotilapia retrodens (Hilgendorf, 1888), Macropleurodus bicolor (Boulenger, 1906) and Platytaeniodus degeni Boulenger, 1906. Haplochromispetronius Greenwood, 1973 (Paperna 1979). Pharyngochromis darlingi (Boulenger, 1911) (Douëllou 1993).

C. tubicirrus: Haplochromis angustifrons Boulenger, 1914, Haplochromis guiarti (Pellegrin, 1904), Haplochromis longirostris (Hilgendorf, 1888), Haplochromis macrops (Boulenger, 1911), Haplochromis obesus (Boulenger, 1906), Haplochromis obliquidens (Hilgendorf, 1888), Haplochromis squamipinnis Regan, 1921, Haplochromis sp., Hoplotilapia retrodens (Hilgendorf, 1888) (original description of C. tubicirrus minutus); Haplochromis aeneocolor Greenwood, 1973, Haplochromis limax Trewavas, 1933, Haplochromis nubilus (Boulenger, 1906), Haplochromis nigripinnis Regan, 1921, Haplochromis elegans Trewavas, 1933, Haplochromis petronius Greenwood, 1973, Tilapia sp. (Paperna 1979).

SITE. — Gills.

AdDitional LOCALITIES. - C. haplochromii: Kazi, Uganda on type host; George Lake, Uganda on Haplochromis wingatii and Haplochromis sp., Kariba Lake, Zimbabwe on Pharyngochromis darlingi (Douëllou 1993).

C. tubicirrus: Georges Lake, Uganda on Haplochromis angustifrons, H. elegans, H. limax, H. macrops, H. nigripinnis, H. petronius, H. schubotzi, H. squamipinnis, Haplochromis sp. (original description of $C$. tubicirrus minutus); Kazinga Channel, Uganda on Haplochromis squamipinnis, H. aeneocolor, North Victoria Lake, Uganda on Haplochromis guiarti, H. longirostris, H. obesus, H. obliquidens, H. nubilus,
Hoplotilapia retrodens, Oreochromis leucostictus, Tilapia sp., Albert Lake, Nabugabo, Bunyoni and Mulehe, Uganda and Ruaha River, Tanzania on Haplochromis spp.

\section{REMARKS}

Paperna (1979) admitted he probably confused this species, according to hosts and locations with $C$. tubicirrus and C. bifurcatus. Douëllou (1993) indicated that $C$. haplochromii represents probably a species complex. Paperna (1979: pl. 1, fig. 7) gave drawings of male copulatory organs as being $C$. dionchus; we think that it is in fact an illustration of $C$. haplochromii genitalia; Paperna in the original description specified neither hosts nor measurements and provided no drawings of haptoral sclerotised parts. The validity of type host, type locality, holotype and paratypes cited by Paperna (1979) for Cichlidogyrus tubicirrus is dubious. In the original description of Cichlidogyrus tubicirrus minutus by Paperna \& Thurston (1969), the figure and measurements correspond exactly to C. haplochromii (cf. Douëllou 1993: 165, 169), at least for specimens from hosts of the genus Haplochromis (like C. haplochromii), which have to be synonymised with C. haplochromii (this concurs with remarks in Douëllou 1993: 169, 170).

\section{Cichlidogyrus hemi Pariselle \& Euzet, 1998}

Cichlidogyrus hemi Pariselle \& Euzet, 1998: 276, fig. 3.

TYPE MATERIAL. - Holotype: MNHN 544 HF Tk75. Paratypes: MNHN 544 HF Tk76, MRAC 37.411.

Type host. - Tilapia brevimanus Boulenger, 1911.

TyPe LOCALITY. — La Ramié, Bourouma River, Guinea.

SITE. — Gills.

Additional localities. - Kogon and Ndyarendi, Kogon River, Guinea.

\section{Cichlidogyrus inconsultans \\ Birgi \& Lambert, 1987}

Cichlidogyrus inconsultans Birgi \& Lambert, 1987: 103. New name for Cichlidogyrus euzeti Birgi \& Lambert, 1986: 525-527, figs 4-6.

TYPE MATERIAL. - No type material mentioned in the original description. 
TyPe HOST. - Polycentropsis abbreviata Boulenger, 1901 (Nandidae).

Type locality. - Mouanko, Cameroon.

SITE. — Gills

\section{REMARKS}

This species was re-named $C$. inconsultans because the name C. euzeti Dossou \& Birgi, 1984 already exists for a parasite from Hemichromis fasciatus.

\section{Cichlidogyrus karibae Douëllou, 1993}

Cichlidogyrus karibae Douëllou, 1993: 175-177, fig. 9. Type MATERIAL. — Holoype (?): MNHN $136 \mathrm{HF}$.

TYPE HOST. - Sargochromis codringtonii (Boulenger, 1908).

Type LOCAlity. — Kariba Lake, Zimbabwe.

ADDITIONAL HOST. - Oreochromis mortimeri (Trewavas, 1966).

SiTE. — Gills.

\section{Cichlidogyrus kothiasi Pariselle \& Euzet, 1994}

Cichlidogyrus kothiasi Pariselle \& Euzet, 1994: 232-234, fig. 4.

TYPE MATERIAL. - Holotype and paratypes: MNHN 212 HF Tk13.

Paratypes: BMNH 1993.3.4.2, MRAC 37.332 and 37.334

TYPE HOST. - Tylochromis jentinki (Steindachner, 1895).

TyPe locality. — Ebrié Lagoon, Côte d'Ivoire.

SITE. — Gills.

Additional locality. - Kogon River, Guinea on type host.

\section{REMARKS}

Pariselle \& Euzet (1994) noticed the unusual morphology of the dorsal transverse bar of Cichlidogyrus from Tylochromis (auricles are continuous with convex face instead of attached on this face); pending new morphological or molecular data from these or other parasites of Tylochromis, they left these species in Cichlidogyrus.

Cichlidogyrus kouassii N'Douba, Thys van den Audenaerde \& Pariselle, 1997

Cichlidogyrus kouassii N'Douba, Thys van den Audenaerde \& Pariselle, 1997: 432, 433, fig. 2.

TYPE MATERIAL. - Holotype: MNHN 523 HF Tk43. Paratypes: MNHN 523 HFTk44, BMNH 1996.10.10.5, MRAC 37.394.

TyPe HOST. - Tilapia guineensis (Bleeker, 1862).

Type Locality. - Bakro, Ayamé Lake, Côte d'Ivoire. SITE. - Gills.

\section{Cichlidogyrus lagoonaris Paperna, 1969}

Cichlidogyrus lagoonaris Paperna, 1969: 861, figs 7377.

Cichlidogyrus gibbus Dossou, 1982: 318, 319, figs 31-33.

TyPe MATERIAL. - C. lagoonaris: Holotype: MRAC M.T. 35.929 (Paperna 1979).

C. gibbus: Holotype: MNHN Tj137; paratype: MNHN 138.

TYPE HOST. - Sarotherodon melanotheron melanotheron Rüppell, 1852.

AdDitional HOst. - Tilapia guineensis (Bleeker, 1862).

TyPe LOCALITY. - C. lagoonaris: Lake Volta, Ghana. C. gibbus: Ouémé and Couffo, Benin.

SITE. — Gills.

REMARKS

We consider that $C$. gibbus, which male apparatus is closely related to that of $C$. lagoonaris, is a junior synonym. Paperna gives Sarotherodon melanotheron heudelotii (Duméril, 1861) as type host for C. lagoonaris coming from Ghana, and yet this fish species is not present in this area (see Paugy et al. 2003), the sub-species from Ghana is $S$. m. melanotheron, which is considered as the type host (as in Dossou 1982 for C. gibbus). 
Cichlidogyrus legendrei Pariselle \& Euzet, 2003

Cichlidogyrus legendrei Pariselle \& Euzet, 2003: 198, fig. 5.

TYPE MATERIAL. - Holotype: MNHN 539 HF Tk65. Paratypes: MNHN 539 HF Tk66, BMNH 1997.1.29.2, MRAC 37.400, CAS M-387.

Type HOST. - Tilapia cabrae Boulenger, 1899.

TyPe LOCALITY. - Cayo Lake, Congo.

SITE. - Gills.

\section{Cichlidogyrus lemoallei Pariselle \& Euzet, 2003}

Cichlidogyrus lemoallei Pariselle \& Euzet, 2003: 198-200, fig. 6.

TYPe Material. - Holotype: MNHN 540 HF Tk67. Paratypes: MNHN 540 HF Tk68, BMNH 1997.1.29.3, MRAC 37.401, CAS M-388.

Type Host. - Tilapia cabrae Boulenger, 1899.

TyPe LOCAlity. - Mouth of Bas Kouilou River, Congo.

SITE. - Gills.

Additional localities. - Loukoula River and Cayo Lake, Congo.

\section{Cichlidogyrus levequei Pariselle \& Euzet, 1996}

Cichlidogyrus levequei Pariselle \& Euzet, 1996: 120, 121, figs 12, 13.

TyPe MATERIAL. - Holotype: MNHN 482 HF Tg92. Paratypes: MNHN 482 HF Tg93, BMNH 1996.1.5.15, MRAC 37.388.

TYPe Host. - Tilapia coffea Thys van den Audenaerde, 1970.

Type LOCAlity. — Niambala, Oulé River, Guinea.

SITE. — Gills.

Additional locality. — Koulé, Guinea.

\section{Cichlidogyrus longicirrus Paperna, 1965}

Cichlidogyrus longicirrus Paperna, 1965: 108, 109, 111.— Dossou \& Birgi 1984: 102-104, figs 1-4.

Type Material. — "Paratype": MRAC M.T. 35.933 (Paperna 1979).
TYPe HOST. — Hemichromis fasciatus Peters, 1857 (Paperna 1979).

TyPe LOCAlity. - New Tafo, Ghana.

Additional Host. - Chromidotilapia guentheri (Sauvage, 1882) (Paperna 1979).

SITE. - Gills.

Additional localities. - Benin and Cameroon on type host (Dossou \& Birgi 1984); Congo on type host (Pariselle \& Euzet 2004).

\section{Cichlidogyrus longipenis}

Paperna \& Thurston, 1969

Cichlidogyrus tubicirrus longipenis Paperna \& Thurston, 1969: 28, fig. 6d, h.

Cichlidogyrus longipenis - Paperna 1979: 13.

TYPE MATERIAL. - “Holotype": private collection of Dr Paperna, no number mentioned.

"Syntype": MRAC M.T. 35.921 (Paperna 1979).

Type host. - Astatoreochromis alluaudi Pellegrin, 1904.

Type LOCAlity. - Jinja, Uganda.

SITE. - Gills.

Cichlidogyrus louipaysani Pariselle \& Euzet, 1995

Cichlidogyrus louipaysani Pariselle \& Euzet, 1995c: 195, fig. 6 .

Type MATERIAL. - Holotype: MNHN 222 HF Tk26. Paratypes: MNHN 222 HF Tk27, BMNH 1993.5.14.11, MRAC 37.341.

TyPe HOST. - Tilapia guineensis (Bleeker, 1862).

TyPe LOCAlity. — Rice field, Koba, Guinea.

SiTE. - Gills.

Additional Localities. - Somone Lagoon, Senegal River, Sine Saloum River, Senegal and Banjul, Gambia River, Gambia.

Cichlidogyrus microscutus Pariselle \& Euzet, 1996

Cichlidogyrus microscutus Pariselle \& Euzet, 1996: 113, 114, figs 4, 5. 
Type MATERial. - Holotype: MNHN 488 HF Tg103. Paratypes: MNHN 488 HFTg104, BMNH 1996.1.5.1011, MRAC 37.385.

TYPe HOST. - Tilapia guineensis (Bleeker, 1862).

TyPe LOCALITY. - Loufoualéba Lake, Congo.

Additional Host. - Tilapia dageti Thys van den Audenaerde, 1971.

SITE. - Gills.

AdDitional LoCAlity. - Niokolo-Koba National Park, Gambia River, Senegal on T. dageti.

\section{Cichlidogyrus nandidae Birgi \& Lambert, 1986}

Cichlidogyrus nandidae Birgi \& Lambert, 1986: 522-524, figs 1-3.

TYPE MATERIAL. - No type material mentioned in the original description..

TyPe HOST. - Polycentropsis abbreviata Boulenger, 1901 (Nandidae).

Type locality. - Mouanko, Cameroon.

SITE. — Gills.

\section{Cichlidogyrus njinei}

Pariselle, Bilong Bilong \& Euzet, 2003

Cichlidogyrus njinei Pariselle, Bilong Bilong \& Euzet, 2003: 207, fig. 5.

Type Material. - Holotype: MNHN 478 HF Tg77. Paratypes: MNHN 478 HF Tg77, MRAC 37.370.

TYPE HOST. - Sarotherodon galilaeus sanagaensis (Thys van den Audenaerde, 1966).

TyPe LOCAlity. — Edéa, Sanaga River, Cameroon.

SITE. - Gills.

\section{Cichlidogyrus nuniezi Pariselle \& Euzet, 1998}

Cichlidogyrus nuniezi Pariselle \& Euzet, 1998: 276-278, fig. 4.

TYPe Material. - Holotype: MNHN 542 HF Tk69. Paratypes: MNHN 542 HFTk70, BMNH 1997.1.30.3, MRAC 37.404.
Type HOST. - Tilapia cessiana Thys van den Audenaerde, 1968.

Type LOCAlity. - Toyebli, Nipoué River, Guinea.

ADDiTIONAL HOST. - Tilapia buttikoferi (Hubrecht, 1881).

SITE. — Gills.

Additional localities. - Kandiafara, Kogon River, Guinea and Katonga, Little Scarcies River, Sierra Leone on T. buttikoferi.

Cichlidogyrus ornatus Pariselle \& Euzet, 1996

Cichlidogyrus ornatus Pariselle \& Euzet, 1996: 115-117, figs 8,9 .

TyPE MATERIAL. - Holotype: MNHN 487HF Tg102. Paratypes: MNHN 487 HF Tg101, BMNH 1996.1.5.1-2, MRAC 37.383.

TYPe HOST. - Tilapia zillii (Gervais, 1848).

TyPe LOCALiTy. - Baoulé River in Côte d'Ivoire.

Additional host. - Tilapia dageti Thys van den Audenaerde, 1971.

SITE. — Gills.

Additional localities. — Bagoué River, Côte d'Ivoire on type host and Comoé River, Côte d'Ivoire on $T$. dageti.

Cichlidogyrus ouedraogoi Pariselle \& Euzet, 1996

Cichlidogyrus ouedraogoi Pariselle \& Euzet, 1996: 121, 122, figs 14,15 .

TYPE MATERIAL. - Holotype: MNHN 483 HF Tg94. Paratypes: MNHN 483 HF Tg95, BMNH 1996.1.5.21, MRAC 37.387.

TYPe HOST. - Tilapia coffea Thys van den Audenaerde, 1970.

TYPE LOCAliTY. — Niambala, Oulé River, Guinea.

SITE. - Gills.

Cichlidogyrus paganoi Pariselle \& Euzet, 1997

Cichlidogyrus paganoi Pariselle \& Euzet, 1997: 226, 227, fig. 5. 
TYPe Material. - Holotype: MNHN 531 HF Tk51. Paratypes: MNHN 531 HFTk52, BMNH 1997.1.28.5, MRAC 37.398.

TYPE HOST. - Sarotherodon occidentalis (Daget, 1962).

Type LOCAlity. — La Ramié, Bourouma River, Guinea.

SITE. - Gills.

Additional localities. - Kamouri, Batapon River, Guinea and Katonga, Little Scarcies River, Sierra Leone.

\section{Cichlidogyrus papernastrema}

Price, Peebles \& Bamford, 1969

Cichlidogyrus papernastrema Price, Peebles \& Bamford, 1969: 120-122, figs 11-20.

TYPE MATERIAL. - No type material mentioned in the original description.

TYPE HOST. - Tilapia sparrmanii Smith, 1840.

Type Locality. - Natal, South Africa.

SITE. — Gills.

Cichlidogyrus philander Douëllou, 1993

Cichlidogyrus philander Douëllou, 1993: 183, 184 , fig. 13.

Type Material. - Holotype: MNHN 137 HF.

Type host. - Pseudocrenilabrus philander philander (Weber, 1897).

Type LOCALITY. — Kariba Lake, Zimbabwe.

SITE. — Gills.

Cichlidogyrus pouyaudi Pariselle \& Euzet, 1994

Cichlidogyrus pouyaudi Pariselle \& Euzet, 1994: 230-232, fig. 3.

Type MATERIAL. - Holotype and paratypes: MNHN 214 HF Tk15.

Paratypes: BMNH 1993.3.4.3, MRAC 37.335.

TYPE HOST. - Tylochromis jentinki (Steindachner, 1895)

TyPe locality. - Ebrié Lagoon, Côte d'Ivoire.

SITE. — Gills:
Additional locality. — Kogon River, Guinea.

\section{REMARKS}

Pariselle \& Euzet (1994) noticed the unusual morphology of the dorsal transverse bar of Cichlidogyrus from Tylochromis (auricles are continuous with convex face instead of attached on this face); pending new morphological or molecular data from these or other parasites of Tylochromis, they left these species in Cichlidogyrus.

\section{Cichlidogyrus quaestio Douëllou, 1993}

Cichlidogyrus quaestio Douëllou, 1993: 181, 182, fig. 12.

TYPe MATeRial. - Holotype: MNHN 137 HF.

TyPe HOST. - Tilapia rendalli (Boulenger, 1897).

TyPe LOCALITY. - Kariba Lake, Zimbabwe.

AdDITIONAL HOSTs. - Sargochromis codringtonii (Boulenger, 1908) and Serranochromis macrocephalus (Boulenger, 1899).

SiTE. - Gills.

\section{REMARKS}

Douëllou (1993) thought that this species is close to C. brevicirrus, and that Thurston (1970) probably confused these two species in Uganda on Tilapia rendalli (Boulenger, 1897).

\section{Cichlidogyrus reversati Pariselle \& Euzet, 2003}

Cichlidogyrus reversati Pariselle \& Euzet, 2003: 196-198, fig. 4.

TYPe Material. - Holotype: MNHN 541 HF Tk69. Paratypes: MNHN 541 HFTk70, BMNH 1997.1.29.4, MRAC 37.402, CAS M-386.

Type host. - Tilapia cabrae Boulenger, 1899.

Type LOCALITY. — Mouth of Bas Kouilou River, Congo.

SITE. - Gills.

\section{Cichlidogyrus rognoni}

Pariselle, Bilong Bilong \& Euzet, 2003

Cichlidogyrus rognoni Pariselle, Bilong Bilong \& Euzet, 2003: 202, 203, fig. 2. 
Type Material. - Holotype: MNHN 536 HF Tk60. Paratypes: MNHN 536 HFTk61, BMNH 1997.2.3.3, MRAC 37.402.

TYPE HOST. - Oreochromis niloticus (Linnaeus, 1758).

TyPe LOCAlity. - Senegal River, Senegal.

SITE. — Gills.

\section{Cichlidogyrus sanjeani Pariselle \& Euzet, 1997}

Cichlidogyrus sanjeani Pariselle \& Euzet, 1997: 227, 228, fig. 6 .

Type Material. - Holotype: MNHN 534 HF Tk56. Paratypes: MNHN 534 HF Tk57, BMNH 1997.1.28.6, MRAC 37.399.

TYPE HOST. - Sarotherodon occidentalis (Daget, 1962).

TyPe LOCALITY. — La Ramié, Bourouma River, Guinea.

SITE. — Gills.

Additional localities. - Kamouri, Batapon River, Guinea and Katonga, Little Scarcies River, Sierra Leone.

Cichlidogyrus sanseoi Pariselle \& Euzet, 2004

Cichlidogyrus sanseoi Pariselle \& Euzet, 2004: 360-362, figs 2,3 .

Type Material. - Holotype: MNHN 236HG TI 233.

Paratypes: MNHN 236HG TI 233 bis, BMNH 2004.9.15.14-15.

Type HOST. - Hemichromis fasciatus Peters, 1858.

TyPe LOCALITY. - Kounougou River, Côte d'Ivoire.

SITE. — Gills.

Additional localities. - Grand Blahoua Lagoon, Côte d'Ivoire; Niokolo-Koba National Park, Gambia River, Senegal; Sélingué, Niger River, Mali.

Cichlidogyrus sclerosus Paperna \& Thurston, 1969

Cichlidogyrus sclerosus Paperna \& Thurston, 1969: 23, 24, fig. 4. — Douëllou, 1993: 164-166, fig. 4.

Cichlidogyrus bangladeshi Ferdousi \& Chandra, 2002: 50-52, fig. 1.
Type Material. - Holotype: MRAC M.T. 34.288 from type host.

Paratype: M.T. 35.576 from hybrid (Paperna 1979); Voucher specimen: MNHN 127 HF (Douëllou 1993).

TYPe Host. - Oreochromis mossambicus (Peters, 1852) (Paperna 1979).

TyPe LOCALITY. — Kajansi, Uganda.

ADDITIONAL HOSTS. - Oreochromis niloticus (Linnaeus, 1758), O. leucostictus (Trewavas, 1933), Haplochromis sp. and Tilapia zillii (Gervais, 1848) (experimental infestation). Hybrid between $O$. mossambicus and O. niloticus, O. spilurus niger (Günther, 1894) and O. aureus (Steindachner, 1864) (Paperna 1979). Oreochromis mortimeri (Trewavas, 1966) and Serranochromis macrocephalus (Boulenger, 1899) (Douëllou 1993).

SITE. - Gills.

Additional localities. - George Lake, Uganda on Oreochromis niloticus and Haplochromis sp.; Jinja, Uganda on O. leucostictus; Kajansi ponds, Uganda on Oreochromis spilurus niger, O. niloticus and Tilapia zillii; Jinja and Victoria Lake, Uganda on O. leucostictus; Fish pounds, Coastal Plain, Israel on $O$. aureus. Kariba Lake, Zimbabwe on $O$. mortimeri and Serranochromis macrocephalus (Douëllou 1993). BFRI fish farm ponds, Bangladesh (on introduced O. mossambicus; Ferdousi \& Chandra 2002).

\section{Cichlidogyrus slembroucki \\ Pariselle \& Euzet, 1998}

Cichlidogyrus slembroucki Pariselle \& Euzet, 1998: 280281, fig. 6 .

Type Material. - Holotype: MNHN 546 HF Tk79. Paratypes: MNHN 546 HF Tk79, BMNH 1997.1.30.4, MRAC 37.405.

TyPE HOST. - Tilapia buttikoferi (Hubrecht, 1881).

Type Locality. - Kandiafara, Kogon River, Guinea.

SITE. - Gills.

\section{Cichlidogyrus testificatus Dossou, 1982}

Cichlidogyrus testificatus Dossou, 1982: 319-321, figs 3436.

Type Material. - Holotype: MNHN Tj 139.

Paratype: MNHN Tj 140. 
Type locality. - Ouémé, Benin.

Type Host. - Tilapia mariae Boulenger, 1899.

SITE. — Gills.

\section{Cichlidogyrus teugelsi Pariselle \& Euzet, 2004}

Cichlidogyrus tengelsi Pariselle \& Euzet, 2004: 362, 363, fig. 4.

TyPe Material. - Holotype: MNHN 237HG TI 234.

Paratypes: MNHN 237HG TI 234 bis, BMNH 2004.9.15.12-13.

TyPe HOST. - Hemichromis fasciatus Peters, 1857.

Type locality. - Kounougou River, Côte d'Ivoire.

SITE. — Gills.

Additional localities. — Adiopodoumé, Côte d'Ivoire; Niokolo-Koba National Park, Gambia River, Senegal and Sélingué, Niger River, Mali.

\section{Cichlidogyrus thurstonae Ergens, 1981}

Cichlidogyrus thurstonae Ergens, 1981: 211, 212, fig. 8. Pariselle et al. 2003: 207-209, fig. 6.

Cichlidogyrus tiberianus Paperna, 1960 individuals from: Sarotherodon galilaeus (Linnaeus, 1758) (in Paperna 1968: 92; 1969: 863); Oreochromis niloticus and Haplochromis longirostris (Hilgendorf, 1888) (in Paperna \& Thurston 1969: 24, 25, fig. 5d left drawing); from Oreochromis variabilis (Boulenger, 1906), O. esculentus (Graham, 1928) and O. niloticus (Linnaeus, 1758) (in Paperna 1979: 14); see Remarks under C. tiberianus.

Cichlidogyrus gilli Ferdousi \& Chandra, 2002: 52-54, fig. 2.

TYPe MATERIAL. - Holotype: CAS, no number mentioned.

Other MATERIAL. - MNHN 537 HF Tk62 (Pariselle et al. 2003).

TYPE HOST. - Oreochromis niloticus (Linnaeus, 1758).

Type locality. - Cairo, Nile River, Egypt.

AdDitional HOST. - Haplochromis longirostris (Hilgendorf, 1888) (Paperna \& Thurston 1969; Ergens 1981). Oreochromis mossambicus (Ferdousi \& Chandra, 2002).
SITE. - Gills.

Additional localities. - Ghana on Sarotherodon galilaeus (Paperna 1968, 1969). Uganda on O. niloticus and Haplochromis longirostris (Paperna \& Thurston 1969) and on Oreochromis variabilis, $O$. esculentus and $O$. niloticus (Paperna 1979). Senegal on type host (Pariselle et al. 2003). BFRI fish farm ponds, Bangladesh (on introduced O. mossambicus; Ferdousi \& Chandra 2002).

\section{REMARKS}

This species was re-described by Pariselle et al. (2003) on the type host in Senegal as Ergens (1981) did not mention, in the original description, the auxiliary plate associated with the male apparatus.

\section{Cichlidogyrus tiberianus Paperna, 1960}

Cichlidogyrus tiberianus Paperna, 1960: 10-12, figs 7, 8. - Dossou 1982: 299-301, figs 4-6. - Douëllou, 1993: 173, 174, fig. 8.

TYPE MATERial. - No type material mentioned in the original description.

TYPe HOST. - Tilapia zillii (Gervais, 1848).

Type LOCALITY. — Sea of Galilee, Israel.

AdDITIONAL HOSTS. - Tristramella simonis simonis (Günther, 1864) and T. sacra (Günther, 1865) (experimental infection only), Haplochromis flaviijosephi (Lortet, 1883). Tilapia rendalli (Boulenger, 1897) (Paperna 1979; Douëllou 1993). Tilapia coffea Thys van den Audenaerde, 1970, Tilapia dageti Thys van den Audenaerde, 1971, T. guineensis (Bleeker, 1862), T. walteri Thys van den Audenaerde, 1968 (Pariselle \& Euzet 1995c, 1996).

SITE. - Gills.

Additional LOCALities. - Southern Ghana on type host (Paperna 1965). Uganda on type host (Paperna \& Thurston 1969). Uganda on T. rendalli (Paperna 1979). Egypt on type host (Ergens 1981). Benin on type host (Dossou 1982). Zimbabwe on T. rendalli (Douëllou 1993). Senegal, Guinea, Côte d'Ivoire and Congo on Tilapia guineensis, T. coffea, T. dageti, T. walteri and type host (Pariselle \& Euzet 1995c, 1996).

\section{REMARKS}

Ergens (1981) in Egypt found and re-described C. tiberianus on Tilapia zillii; he indicated that C. tiberianus on Oreochromis niloticus and Haplo- 
chromis longirostris found in Uganda by Paperna \& Thurston (1969) (with some differences with original descriptions from Ghana or Israel) had to be considered as C. thurstonae. Dossou (1982) in Benin found C. tiberianus on Tilapia zillii, he agreed with Ergens (1981) about the confusion made by Paperna \& Thurston (1969) between C. tiberianus and C. thurstonae. Douëllou (1993), according to Ergens (1981), considered that Paperna \& Thurston (1969) confused $C$. tiberianus with $C$. thurstonae; she added that Paperna (1968, 1969 and 1979) may have made the same error. Pariselle \& Euzet (1995c, 1996) found C. tiberianus only from hosts of the sub-genus Coptodon (namely: Tilapia coffea, T. dageti, T. guineensis, T. walteri and T. zillii). All these remarks are leading us to the same conclusion: all individuals described as $C$. tiberianus from mouthbreeders hosts (genus Oreochromis and Sarotherodon) have been confused with $C$. thurstonae by Paperna $\&$ Thurston (1969) or Paperna (1968, 1969 and 1979).

\section{Cichlidogyrus tilapiae Paperna, 1960}

Cichlidogyrus tilapiae Paperna, 1960: 5-9, figs 3-6. Douëllou 1993: 167, 168, fig. 5.

Cleidodiscus tilapiae - Price 1967: 381. — Paperna 1979: 14.

Cichlidogyrus tubicirrus minutus Paperna \& Thurston, 1969: 26-28, fig. 6c, g. — Paperna 1979: 14 (individuals from Oreochromis leucostictus).

Cichlidogyrus tubicirrus - Paperna 1979: 14 (individuals from Oreochromis leucostictus).

Cichlidogyrus chandrai Ferdousi \& Chandra, 2002: 54-56, fig. 3.

Type MATERIAL. - Of C. tilapiae: no type material mentioned in the original description.

Of C. tubicirrus (from Oreochromis leucostictus): Paratype: MRAC M.T. 35932 (Paperna 1979).

TYPE HOST. - Sarotherodon galilaeus (Linnaeus, 1758) (Paperna 1979).

Type LOCALiTy. - Jordan and coastal system, Israel (Paperna 1979).

Other Material. - C. tilapiae: MNHN $130 \mathrm{HF}$ (Douëllou 1993).
ADDITIONAL HOSTS. - Oreochromis niloticus (Linnaeus, 1758), Tristramella sacra (Günther, 1865) and Tristramella simonis (Günther, 1864) (experimental infestation). Tilapia busumana (Günther, 1903) and Hemichromis fasciatus Peters, 1857 (Paperna 1968). Oreochromis leucostictus (Trewavas, 1933), Oreochromis mossambicus (Peters, 1852), Tilapia zillii (Gervais, 1848) (experimental infestation) and Haplochromis macrognathus Regan, 1922 (Paperna \& Thurston 1969). Chromidotilapia guentheri (Sauvage, 1882) (Paperna 1969). Oreochromis aureus (Steindachner, 1864), Oreochromis niloticus vulcani (Trewavas, 1933), Oreochromis spilurus niger (Günther, 1894), Oreochromis variabilis (Boulenger, 1906) and Oreochromis urolepis urolepis (Norman, 1922) (Paperna 1979). Oreochromis mortimeri (Trewavas, 1966) (Douëllou 1993).

SITE. — Gills.

Additional localities. - Dor, Fish Culture Research Station, Israel on Oreochromis niloticus; Ginossar, Fisheries Field Station, Israel on Sarotherodon galilaeus; Western shores, Sea of Galilee, Israel on Tristramella sacra. Southern Ghana on O. niloticus and S. galilaeus (Paperna 1965). Ghana on Tilapia busumana and Hemichromis fasciatus (Paperna 1968). Uganda on Oreochromis leucostictus, O. mossambicus, Tilapia zillii (experimental infestation) and Haplochromis macrognathus (Paperna \& Thurston 1969). Volta Basin, South Ghana on Sarotherodon galilaeus, Oreochromis niloticus and Chromidotilapia guentheri (Paperna 1979). Egypt on Oreochromis niloticus and Tilpia zillii (Ergens 1981). Kariba Lake, Zimbabwe on Oreochromis mortimeri (Douëllou 1993). Bangladesh in $\mathrm{BFRI}$ and BAU fish farm ponds on introduced $O$. niloticus and O. mossambicus (Ferdousi \& Chandra 2002).

\section{REMARKS}

Paperna (1979) synonymised Cichlidogyrus tubicirrus minutus with Cichlidogyrus tubicirrus, the validity of type host, type locality, holotype and paratypes given in this paper is dubious; in the original description by Paperna \& Thurston (1969), the figure and measurements of specimens from Oreochromis leucostictus, are almost identical to those of $C$. tilapiae (cf. Ergens 1981: 210), with which C. tubicirrus (from O. leucostictus) has to be synonymised.

\section{Cichlidogyrus vexus Pariselle \& Euzet, 1995}

Cichlidogyrus vexus Pariselle \& Euzet, 1995: 195-197, fig. 7.

Type MATERIAL. - Holotype: MNHN 219 HF Tk20. Paratypes: MNHN 219 HFTk21, BMNH 1993.5.14.12, MRAC 37.339. 

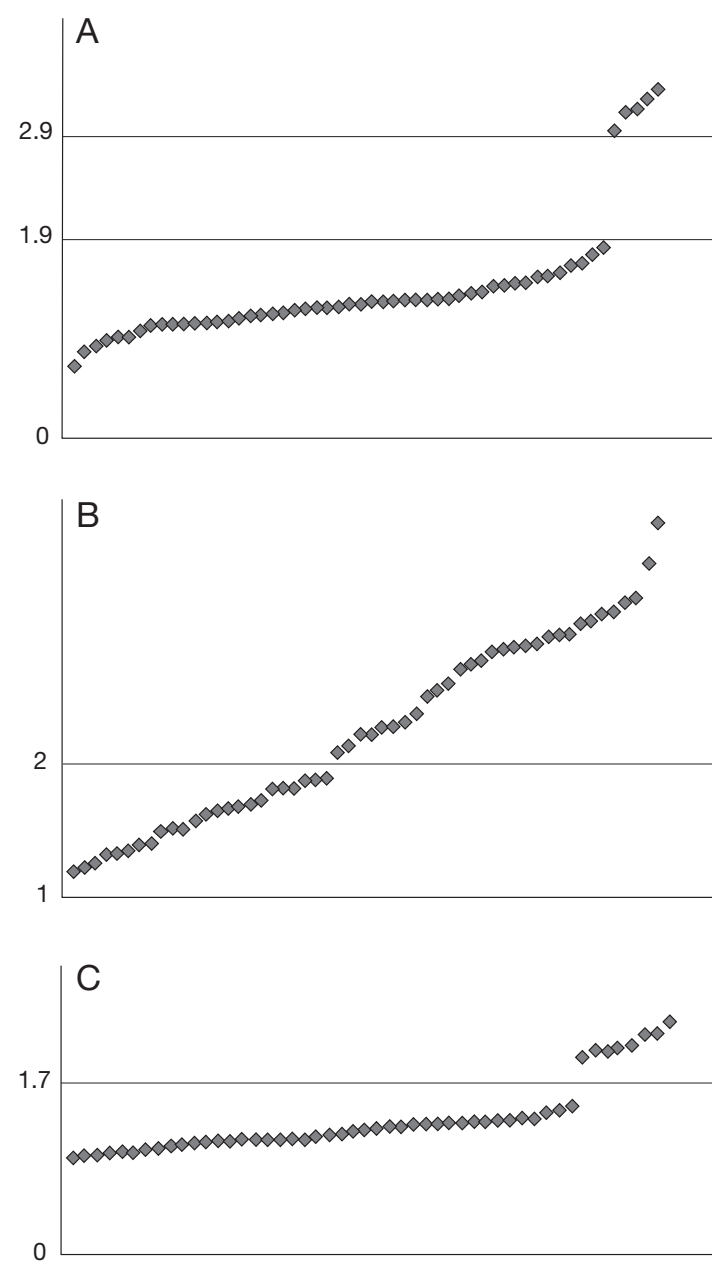

FIG. 1. - Definition of characters used in the identification keys of Cichlidogyrus Paperna, 1960 species (from 54 species studied): A, value repartition of the standardised length of dorsal transverse bar auricles; B, value repartition of the standardised length of uncinuli pairs III-VII; C, value repartition of the standardised length of uncinuli pair I.

Type locality. - Tiassalé, Bandama River, Côte d'Ivoire.

Type HOST. - Tilapia guineensis (Bleeker, 1862).

Additional host. - Tilapia zillii (Gervais, 1848).

SITE. - Gills.

Additional LOCAliTies. — Layo Research Station, Ebrié Lagoon, Côte d'Ivoire on type host; Kossou Lake, Côte d'Ivoire on type host and T. zillii. Senegal, Guinea and Congo on type host (Pariselle \& Euzet 1996).
Cichlidogyrus yanni Pariselle \& Euzet, 1996

Cichlidogyrus yanni Pariselle \& Euzet, 1996: 118-120, fig. 11.

TYPe MATERIAL. - Holotype: MNHN 481 HF Tg90. Paratypes: MNHN 481 HF Tg91, BMNH 1996.1.5.2225, MRAC 37.386.

Type HOST. - Tilapia zillii (Gervais, 1848).

TyPe LOCAlity. - Kogon River, Guinea.

Additional hosts. - Tilapia dageti Thys van den Audenaerde, 1971, T. guineensis (Bleeker, 1862), T. louka Thys van den Audenaerde, 1969, and T. walteri Thys van den Audenaerde, 1968.

SITE. - Gills.

Additional localities. — Volta Noire River, Burkina Faso on type host; Niokolo-Koba National Park, Gambia River, Senegal on T. dageti; Layo Research Station, Ebrié Lagoon, Côte d'Ivoire and Senegal River, Senegal on T. guineensis; Bourouma River, Guinea on T. louka; Cavally and Nipoué Rivers, Côte d'Ivoire on T. walteri.

Cichlidogyrus zambezensis Douëllou, 1993

Cichlidogyrus zambezensis Douëllou, 1993: 178-181, fig. 11.

TyPe MATERIAL. - Holotype: MNHN 138 HF.

TYPE HOST. - Serranochromis macrocephalus.

ADDITIONAL HOST. - Oreochromis mortimeri (Trewavas, 1966).

Type Locality. - Kariba Lake, Zimbabwe.

SiTE. — Gills.

\section{IDENTIFICATION KEY FOR STUDIED GENERA AND SPECIES}

Following Dossou (1985), Pariselle \& Euzet (1995c) and Pariselle (1996) the 71 species forming Cichlidogyrus, recorded from 66 different host species (64 Cichlidae, one Cyprinodontidae, one Nandidae), may be clustered in different groups according to the relative size of their haptoral sclerites. This has been confirmed by Pouyaud et al. (2006) 
using both genetic and morphometrical data: "This division into four groups based on morphological criterions is also confirmed by the genetic distances". These groups can be easily characterised by morphometrical characters: length of the transverse dorsal bar auricles, of the uncinulus pairs III-VII, and of uncinuli pairs I.

The following may introduced morphometrical differences into the size range for these characters: developmental stage of the examined parasite; influence of temperature on the size of the sclerites (Ergens \& Gelnar 1985; Appelby 1996); equipment used; method by which the measurements are taken, or other constraints (see Caltran et al. 1995). To avoid these problems, the length of each examined sclerite, according to Pariselle \& Euzet (2003), will be "standardised" by dividing its total length by the total length of uncinuli pair II, which retain its size from larval to adult stages of development in ancyrocephalid Monogenea.

- Length of transverse dorsal bar auricles: the distribution of this character values (Fig. 1A) shows a clear gap between species belonging to Scutogyrus (> 2.9) (Fig. 2E) and Cichlidogyrus (<1.9) (Fig. 2F). This unequivocal character, therefore, is relevant to diagnose Scutogyrus.

- Size of uncinuli pairs III-VII: the gap between large and small standardized sizes of uncinuli pairs III-VII is located near 2 (Fig. 1B).

- Size of uncinuli pair I: the gap between large and small standardized sizes of uncinuli pairs I is located near 1.7 (Fig. 1C).

1. One transverse bar (ventral)

— More than one transverse bar (ventral and dorsal) .................................................... 3

2. One asymmetrical pair of anchors (ventral) (Fig. 2A)

Urogyrus

- Two pairs of symmetrical anchors (Fig. 2B) 6 Enterogyrus

3. Two transverse bars (one dorsal and one ventral) ....................................................... 4

— Three transverse bars (one dorsal, two ventral) (Fig. 2C) ..................... 15 Onchobdella

4. Dorsal bar with wings (= auricles) (Fig. 2D, a) ........................................................ 5

— Dorsal bar lacking wings (Fig. 2D, b) ............................................... 13 Insulacleidus

5. Dorsal bar winged with very long auricles, ventral bar associated with a plate (Fig. 2E)

- Dorsal bar with short auricles ventral bar not associated with a plate (Fig. 2F) 22 Scutogyrus 27 Cichlidogyrus

6. Dorsal anchor total length more than $50 \mu \mathrm{m}$, ventral bar straight, more than $40 \mu \mathrm{m}$ (Fig. 3A, a)

- Dorsal anchor total length less than $50 \mu \mathrm{m}$, ventral bar V-shaped, less than $40 \mu \mathrm{m}$ (Fig. 3A, b)

Enterogyrus malmbergi

7. Ventral anchor total length less than $27 \mu \mathrm{m}$ Enterogyrus crassus

8. Dorsal anchor with shaft longer than blade (Fig. 3B, a)

- Dorsal anchor with shaft shorter than blade (Fig. 3B, b)

9. Haptor cup-shaped Enterogyrus cichlidarum

- Haptor in two parts: one bulbous and one peduncular shaped (Fig. 3C) .................. 10

10. Penis with distal turns well separated (Fig. 3D, a)

— Penis with distal turns adjoining (Fig. 3D, b) Enterogyrus foratus 
11. Ventral bar total length less than $14 \mu \mathrm{m}$ Enterogyrus amieti

- Ventral bar total length more than $16 \mu \mathrm{m}$ 12

12. Penis with 3-2-3 turns (Fig. 3E, a) Enterogyrus melenensis

- Penis with 6-2-4 turns (Fig. 3E, b) Enterogyrus barombiensis

13. Looped penis (Fig. 4B, a)

- Straight penis (Fig. 4B, b) Insulacleidus paratilapiae

14. Simple accessory piece (Fig. 4C, a)

- Accessory piece complex (Fig. 4C, b) Insulacleidus paretropli

15. Dorsal bar clearly arched (M-shaped) (Fig. 5A, a) 16

- Dorsal bar straight or slightly V-shaped (Fig. 5A, b) 19

16. Ventral bars longer than dorsal one (Fig. 5B, a)

- Ventral bars shorter than dorsal one (Fig. 5B, b) Onchobdella pterigyalis

17. Penis J-shaped (Fig. 5C, a) Onchobdella voltensis

- Penis long and sinuous or spirally coiled (Fig. 5C, b) 18

18. Penis sinuous, no visible vagina (Fig. 5D, a)

- Penis spirally coiled ( 4 to 5 turns), long and sinuous vagina (Fig. 5D, b)

Onchobdella spirocirra Onchobdella bopeleti

19. Dorsal bar slightly V-shaped, length more than $43 \mu \mathrm{m}$ (Fig. 5E, a) ... Onchobdella silverai

- Dorsal bar straight, length less than $40 \mu \mathrm{m}$ (Fig. 5E, b) 20

20. Ventral anchor total length less than $11 \mu \mathrm{m}$ (Fig. 5F, a) Onchobdella melissa

- Ventral anchor total length more than $20 \mu \mathrm{m}$ (Fig. 5F, b) 21

21. Penis thin, G-shaped, total length $80 \mu \mathrm{m}$ (Fig. 5G, a) Onchobdella krachii

- Penis large, J-shaped, total length less than $60 \mu \mathrm{m}$ (Fig. 5G, b) .... Onchobdella aframae

22. Penis and vagina very long and thin (Fig. 6A, a) Scutogyrus ecoutini

— Penis and vagina shorter (Fig. 6A, b) 23

23. Penis length more than $70 \mu \mathrm{m}$ (Fig. 6B, a) 24

— Penis length less than $70 \mu \mathrm{m}$ (Fig. 6B, b) 25

24. Vagina tubular, thin-walled (Fig. 6C, a)

- Vagina large, thick-walled (Fig. 6C, b) Scutogyrus bailloni Scutogyrus gravivaginus

25. Accessory piece ending in two curved pincer-like hooks (Fig. 6D, a) ... Scutogyrus chikhii

- Accessory piece ending in pincers with one component hooked, the other blunt (Fig. 6D, b) 26

26. Accessory piece basal extension well marked (Fig. 6E, a)

- No accessory piece extension (Fig. 6E, b) Scutogyrus minus

27. Uncinuli pairs III-VII long ("standardised length" more than 2) (Fig. 7A, a) ............ 28

— Uncinuli pairs III-VII short ("standardised length" less than 2) (Fig. 7A, b) ............... 53

28. Uncinuli pairs I large ("standardised length" more than 1.7) (Fig. 7B, a) .................. 29

— Uncinuli pairs I small ("standardised length" less than 1.7) (Fig. 7B, b) 30 
A

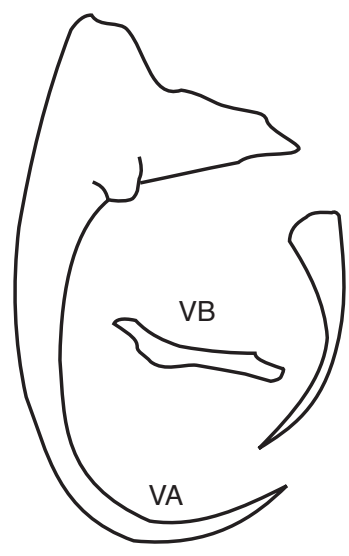

B
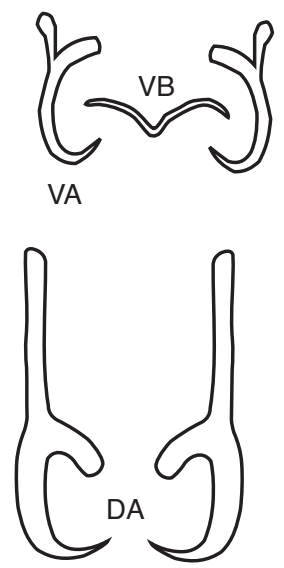

C
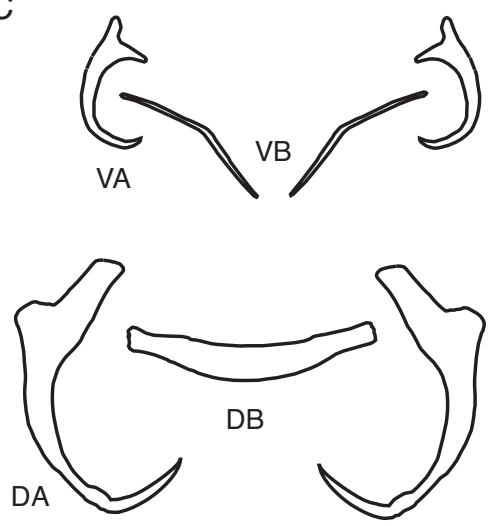

D
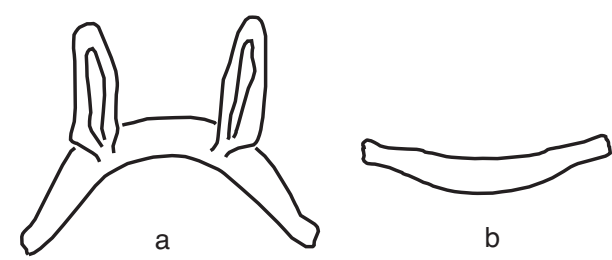

b

E
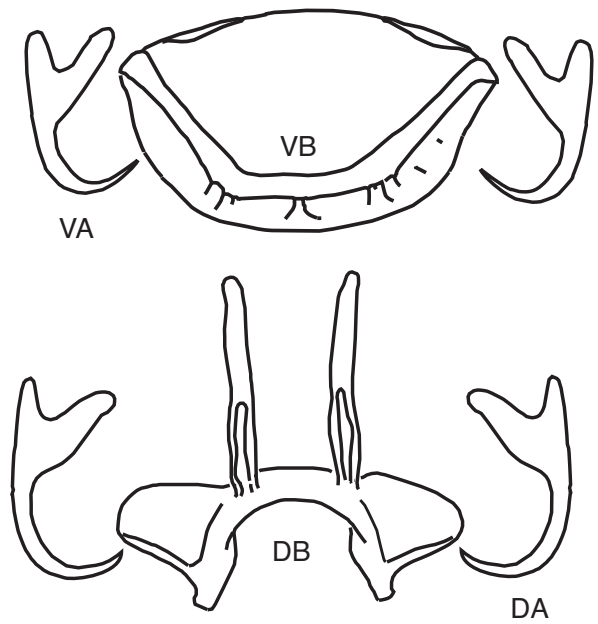

F
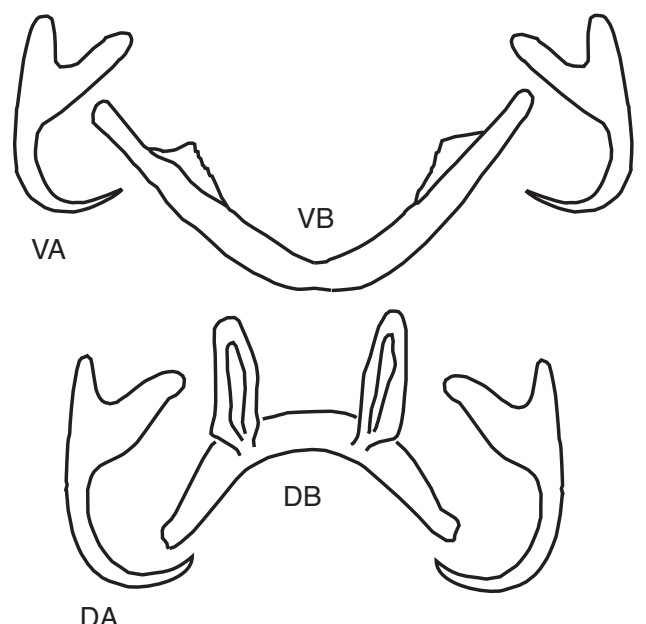

FIG. 2. - Illustration of the identification key for studied genera: A, haptoral morphology of Urogyrus Bilong Bilong, Birgi \& Euzet, 1994 species; B, haptoral morphology of Enterogyrus Paperna, 1963 species; C, haptoral morphology of Onchobdella Paperna, 1968 species; D, shape of dorsal bars (not winged [a], winged [b]); E, haptoral morphology of Scutogyrus Pariselle \& Euzet, 1995 species; F, haptoral morphology of Cichlidogyrus Paperna, 1960 species. Abbreviations: DA, dorsal anchor; DB, dorsal transverse bar; VA, ventral anchor; VB, ventral transverse bar. 
A

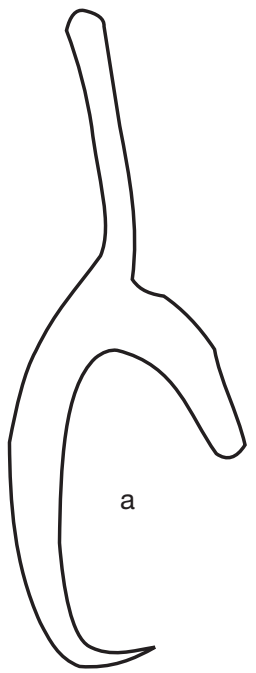

C

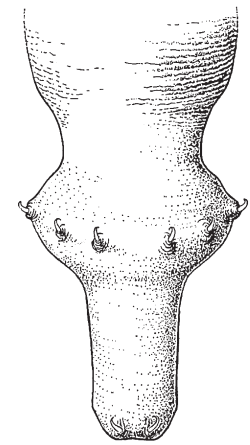

$\mathrm{D}$

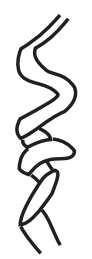

a
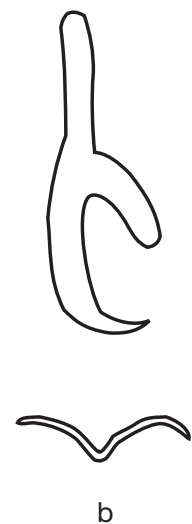

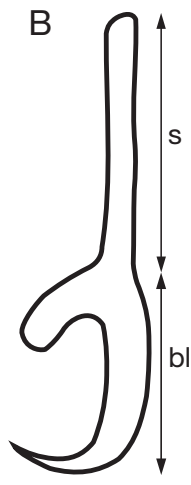

a

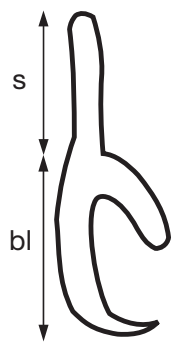

b

E

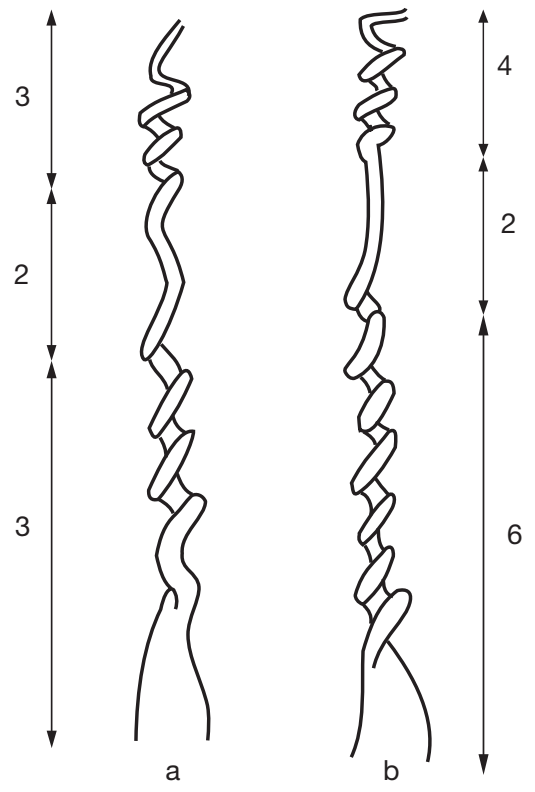

FIG. 3. - Illustration of identification key for Enterogyrus Paperna, 1963 species: A, size of dorsal anchor (large [a], small [b]) and shape of dorsal transverse bar (straight [a], V-shaped [b]); B, relative length of dorsal anchor blade and shaft (long shaft [a], short shaft [b]); $\mathbf{C}$, morphology of haptor with bulbous and peduncular parts; $\mathbf{D}$, penis distal turns (separated [a] or adjoining [b]); $\mathbf{E}$, number of turns in the different parts of the penis. Abbreviations: bl, blade; s, shaft; 2, 3, 4 and 6, number of turns in the parts of the penis. 

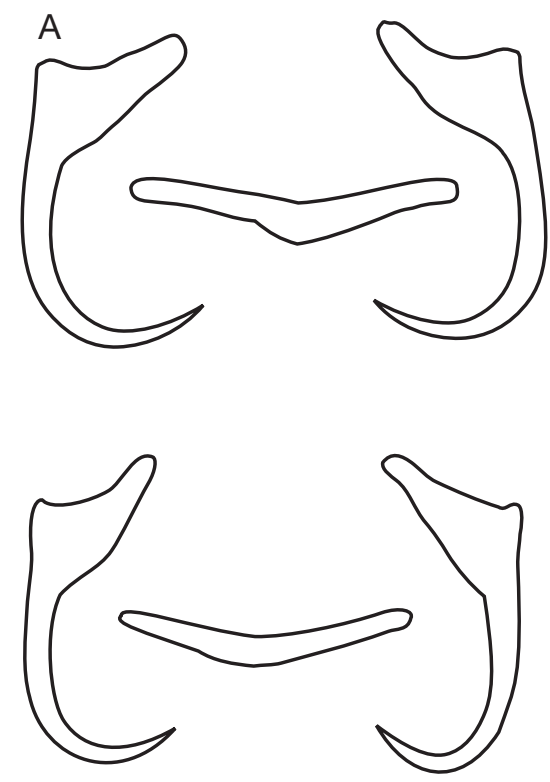

$\mathrm{B}$

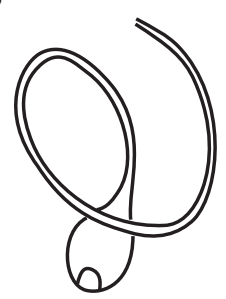

a

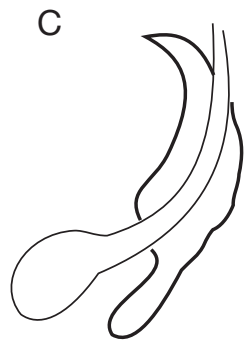

a

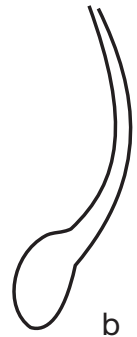

b

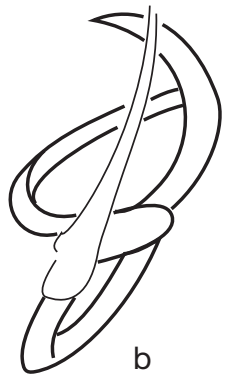

FIG. 4. - Illustration of identification key for Insulacleidus Rakotofiringa \& Euzet, 1983 species: A, haptor morphology; B, shape of the penis (looped [a], straight [b]); C, shape of the accessory piece (simple [a], complex [b]). 

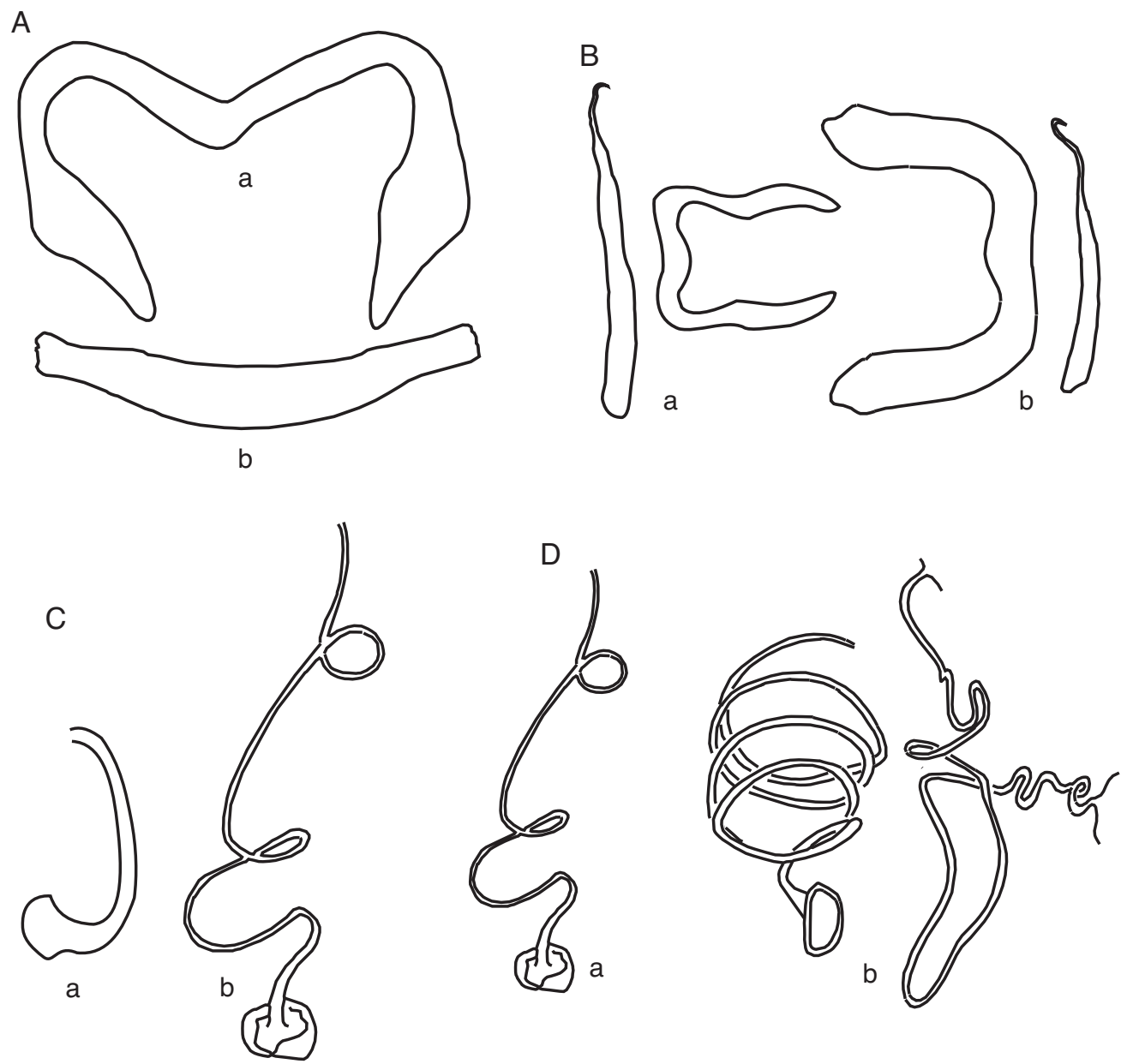

E
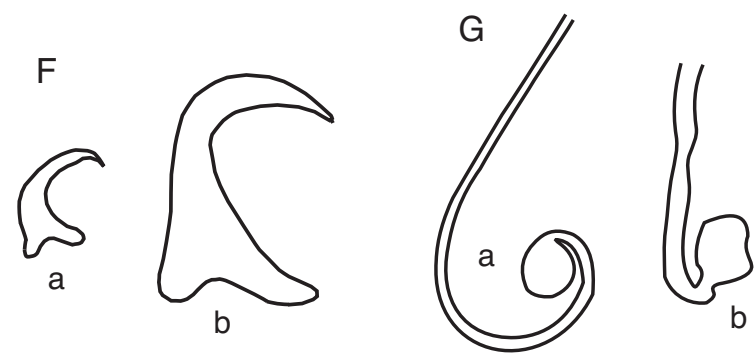

FIG. 5. - Illustration of identification key for Onchobdella Paperna, 1968 species: A, shape of dorsal transverse bar (arched [a], straight or V-shaped [b]); B, relative length of dorsal and ventral bars (ventral > dorsal [a], ventral < dorsal [b]); C, shape of penis (J-shaped [a], sinuous or spirally coiled [b]); $\mathbf{D}$, shape of penis (sinuous [a], spirally coiled [b]); $\mathbf{E}$, shape and size of dorsal bar (long and V-shaped [a], short and straight [b]); F, size of ventral anchor (small [a], large [b]); G, shape of the penis (G-shaped [a], J-shaped [b]). 
A

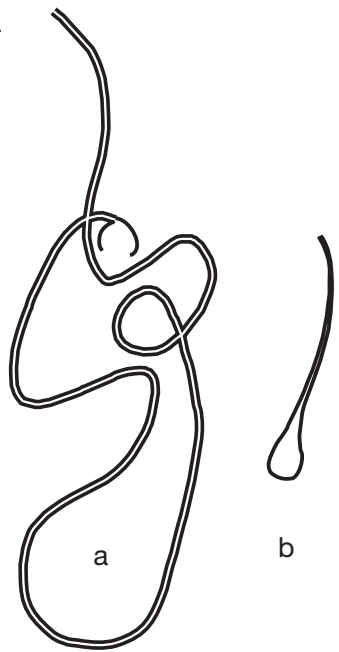

C

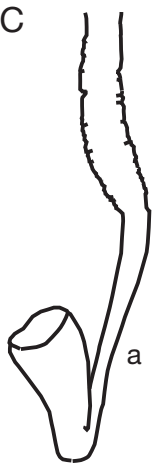

B
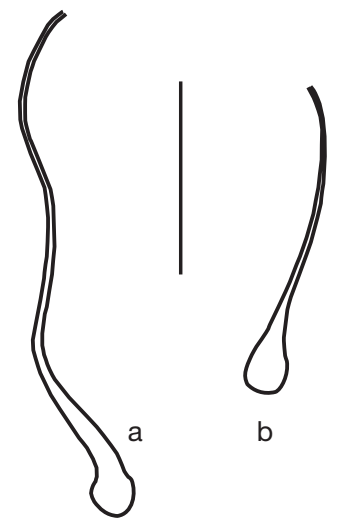

$\mathrm{D}$

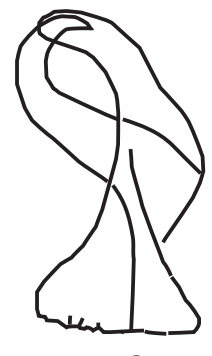

a

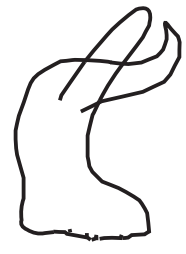

b

$E$
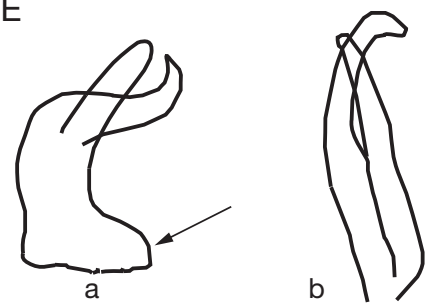

FIG. 6. - Illustration of identification key for Scutogyrus Pariselle \& Euzet, 1995 species: A, size of the penis (long [a], short [b]); B, size of the penis (long [a], short [b]); C, shape of the vagina (tubular and thin-walled [a], large and thick-walled [b]); $\mathbf{D}$, shape of the accessory piece ending (two curved pincer-like hooks [a], one component hooked, the other blunt [b]); $\mathbf{E}$, shape of the accessory piece (basal extension well marked [a], no extension [b]). Scale bar: $30 \mu \mathrm{m}$. 
29. Vagina not visible, single-looped penis (Fig. 7C, a) Cichlidogyrus arthracanthus

- Sclerotised vagina, curved penis (Fig. 7C, b) Cichlidogyrus inconsultans

30. Very large body and sclerite size, large and S-shaped penis (Fig. 7D, a) ... Cichlidogyrus halli

— Other penis shapes (Fig. 7D, b) ................................................................................. 31

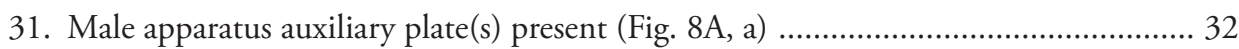

- Male apparatus without auxiliary plate (Fig. 8A, b) ................................................. 39

32. Presence of two small auxiliary plates (Fig. 8B, a) ......................... Cichlidogyrus guirali

— Presence of one auxiliary plate (Fig. 8B, b) ................................................................ 33

33. Reduced auxiliary plate (smallest axis $<10 \mu \mathrm{m}$ ) (Fig. 8C, a) ... Cichlidogyrus microscutus

— Large auxiliary plate (smallest axis $>10 \mu \mathrm{m})$ (Fig. 8C, b) .......................................... 34

34. Accessory piece slightly S-shaped, ending bottle-opener-like (Fig. 8D, a)

— Accessory piece bent at right angle (Fig. 8D, a) ...................................................... 35

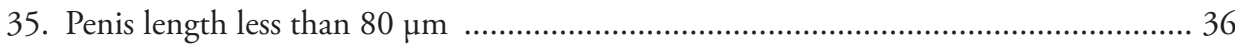

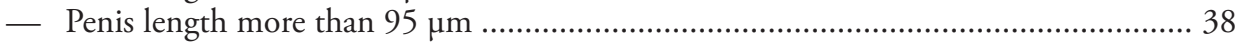

36. Extremity of accessory piece with large perpendicular diverticles, vagina thin walled and amphora-shaped (Fig. 8E, a) ................................................. Cichlidogyrus aegypticus

— Extremity of accessory piece without large perpendicular diverticles (Fig. 8E, b) ...... 37

37. Accessory piece convex face with large semi-circular extension (Fig. 8F, a)

- Accessory piece convex face without large semi-circular extension (Fig. 8F, b)

Cichlidogyrus gallus

Cichlidogyrus paganoi

38. Penis length less than $105 \mu \mathrm{m}$, vagina length less than $50 \mu \mathrm{m}$ C. bilongi

— Penis length more than $130 \mu \mathrm{m}$, vagina length more than $60 \mu \mathrm{m}$ C. agnesi

39. Long and narrow vagina (Fig. 9A, a) 40

— Large and/or short vagina (Fig. 9A, b) 42

40. Penis length more than $85 \mu \mathrm{m}$, Cichlidogyrus flexicolpos

— Penis length less than $60 \mu \mathrm{m}$ 41

41. Accessory piece T-shaped (Fig. 9B, a)

Cichlidogyrus testificatus

- Accessory piece ending in a hook (Fig. 9B, b) Cichlidogyrus lemoallei

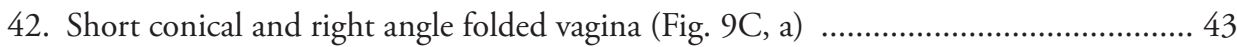

— Tubular vagina (almost constant diameter) (Fig. 9C, b) ............................................. 46

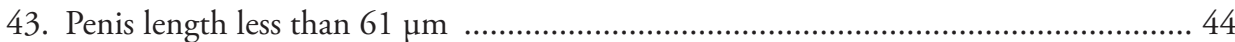

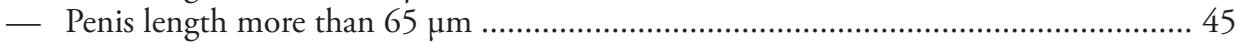

44. Accessory piece ending with smooth convex face (Fig. 9D, a) ........ Cichlidogyrus bouvii - Accessory piece ending in a bifurcated hook with crenulated convex face (Fig. 9D, b) ... Cichlidogyrus dossoui

45. Accessory piece ending folded back (Fig. 9E, a) Cichlidogyrus vexus

- Accessory piece ending in a hook (Fig. 9E, b) Cichlidogyrus douellouae 
III-VII

a

C

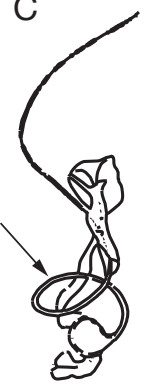

a
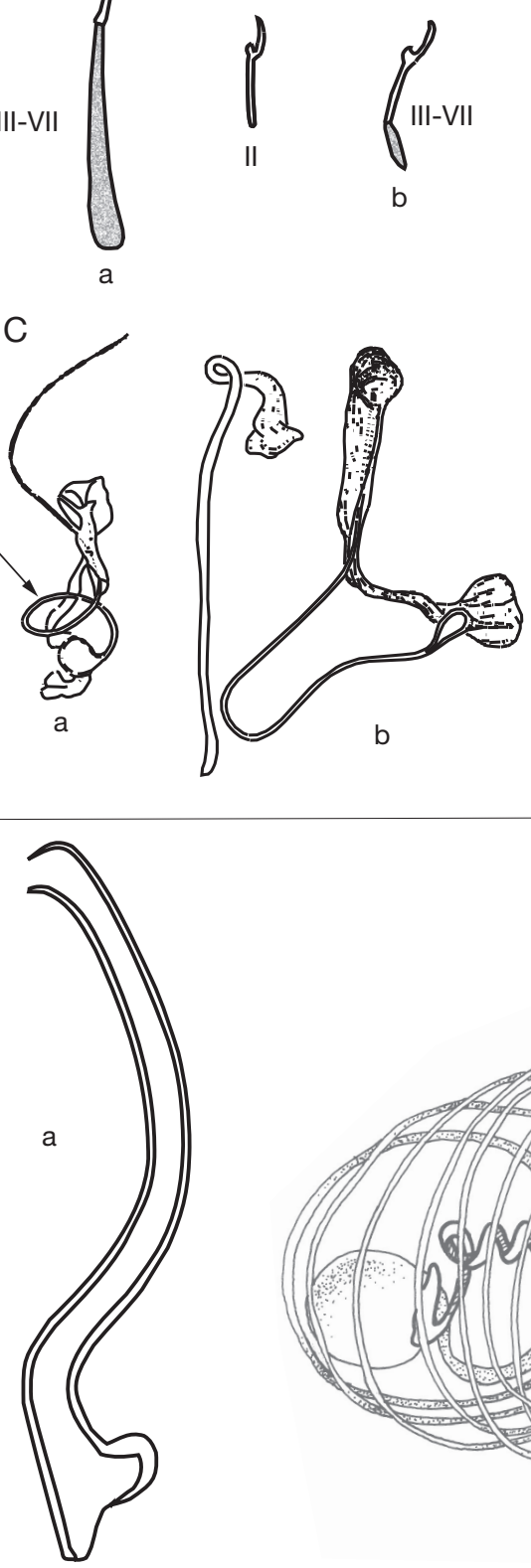

B

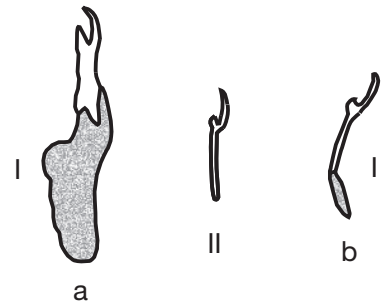

D

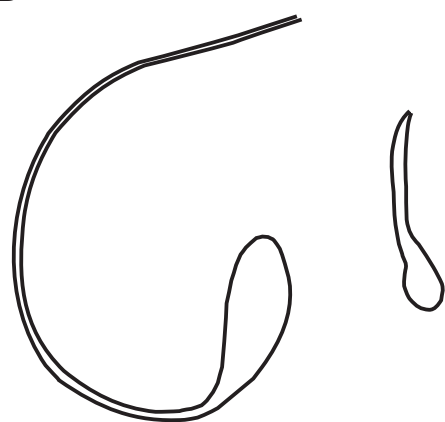

b

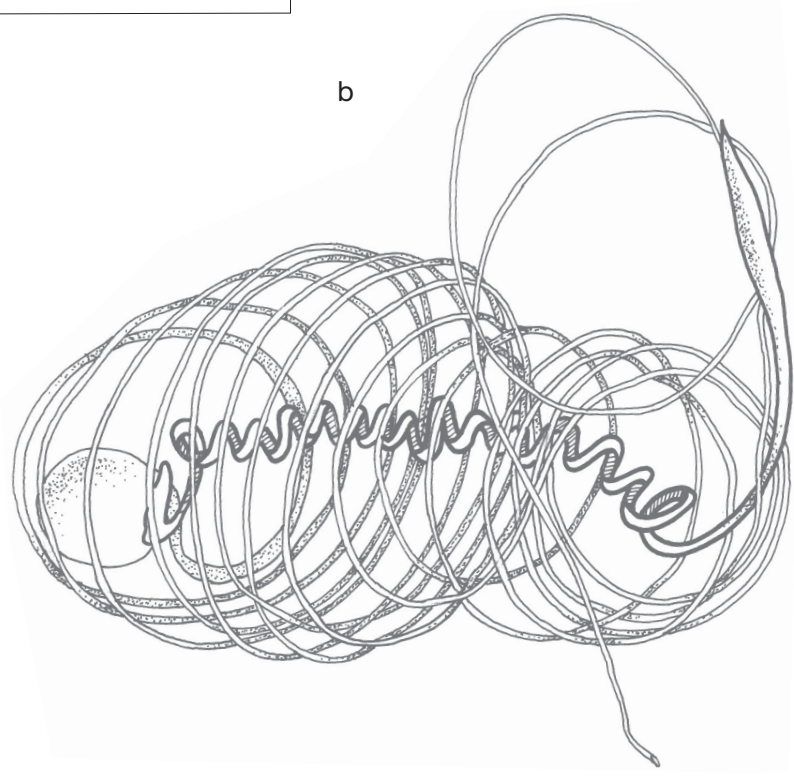

FIG. 7. - Illustration of identification key for Cichlidogyrus Paperna, 1960 species: A, length of uncinuli III to VII (long [a], short [b], pair II is given as a reference); B, size of uncinuli I (large [a], small [b], pair II is given as a reference); C, C. arthracanthus Paperna, 1960 penis (a); C. inconsultans (Birgi \& Lambert, 1986) sclerotised genitalia (b); D, shape of penis (C. halli (Price \& Kirk, 1967) [a], other species [b]). Abbreviations: I, II and III-VII, uncinuli numbering. 
46. Short annulated vagina (Fig. 9F, a)

47. Straight or slightly curved vagina (Fig. 10A, a)

- Well curved or looped vagina (Fig. 10A, b) 48

48. Looped vagina (Fig. 10B, a)

- Curved vagina (not looped) (Fig. 10B, b)

49. Vagina forming a single loop (Fig. 10C, a)

Cichlidogyrus tiberianus

— Vagina forming two semi-loops (Fig. 10C, b) 50

50. Vagina semi-loops in the same plane, crenulated distal accessory piece extremity (Fig. 10D, a)

- Vagina semi-loops in perpendicular planes, accessory piece distal extremity with a single hook (Fig. 10D, b) Cichlidogyrus bonhommei

51. Curved accessory piece ending in two points, vagina with characteristic enlargement (Fig. 10E, a) .................................................................. Cichlidogyrus anthemocolpos

- Accessory piece bent at right angle, ending in a single point, vagina without enlargement (Fig. 10E, b) 52

52. Accessory piece with triangular extension at proximal third (Fig. 10F, a)

- Accessory piece without triangular extension at proximal third (Fig. 10F, b)

Cichlidogyrus ergensi

Cichlidogyrus ouedraogoi

53. Auricles reduced, in continuity with dorsal bar anterior face (Fig. 11A, a) ............... 54

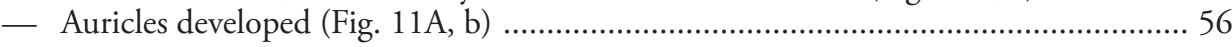

54. Uncinulus I "standardised length" more than 2 (Fig. 11B, a) ....... Cichlidogyrus kothiasi

— Uncinulus I "standardised length" less than 2 (Fig. 11B, b) .................................... 55

55. Accessory piece with narrow base, penis with large heel (Fig. 11C, a) ... Cichlidogyrus berrebii

- Accessory piece with wide base, penis with thin heel (Fig. 11C, b) ... Cichlidogyrus pouyaudi

56. Uncinulus I "standardised length" more than 2 (Fig. 11D, a) .................................. 57

— Uncinulus I "standardised length" less than 2 (Fig. 11D, b) ..................................... 76

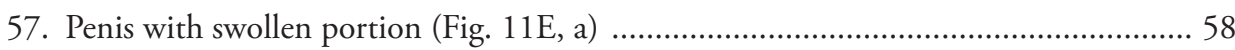

— Penis without swollen portion (thin and tubular all along) (Fig. 11E, b) .................. 59

58. Penis length less than $35 \mu \mathrm{m}$

- Penis length more than $45 \mu \mathrm{m}$ Cichlidogyrus papernastrema

59. Penis length less than $50 \mu \mathrm{m}$ 60

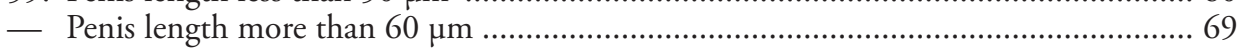

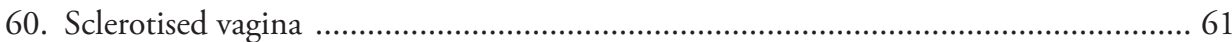

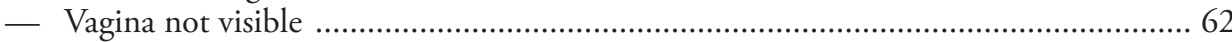

61. Penis with developed heel, accessory piece S-shaped (Fig. 11F, a) ... Cichlidogyrus dageti

- Penis with poorly developed heel, accessory piece C-shaped (Fig. 11F, b) 


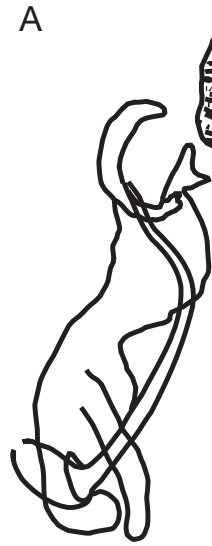

a

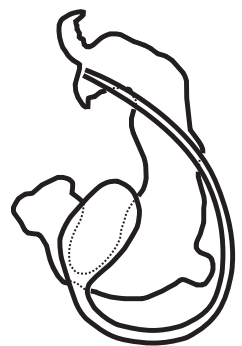

$\mathrm{b}$

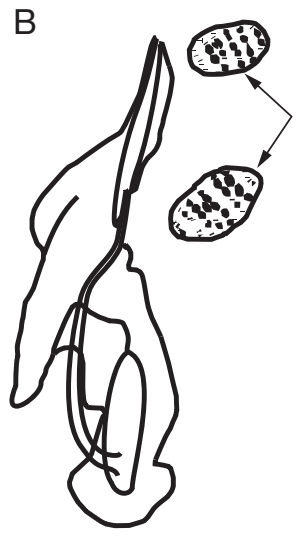

a

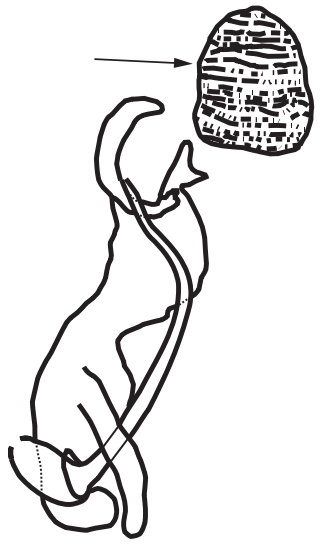

b
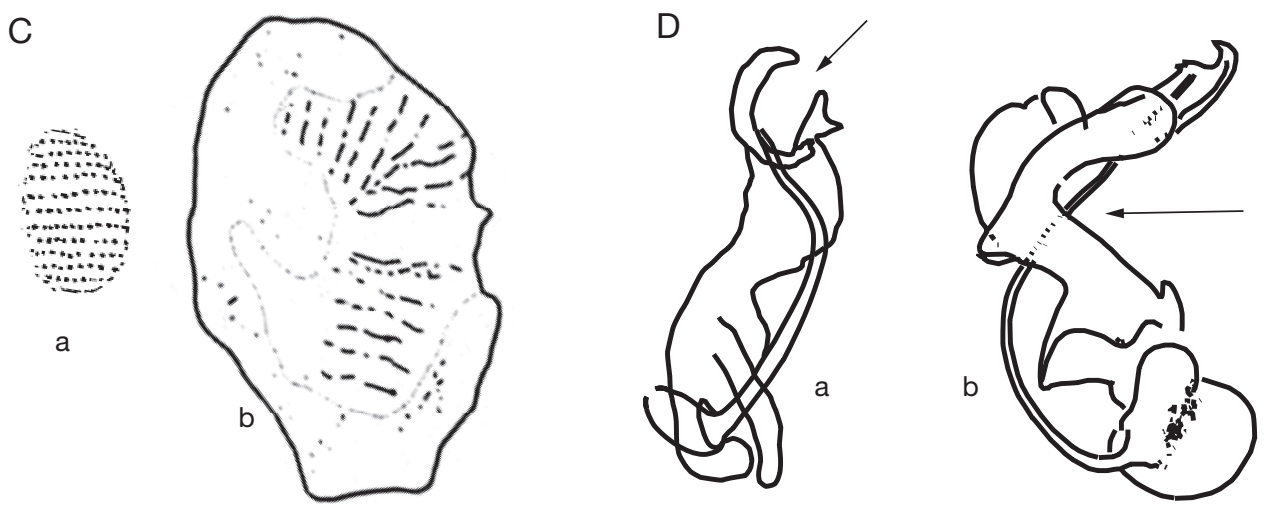

$\mathrm{E}$
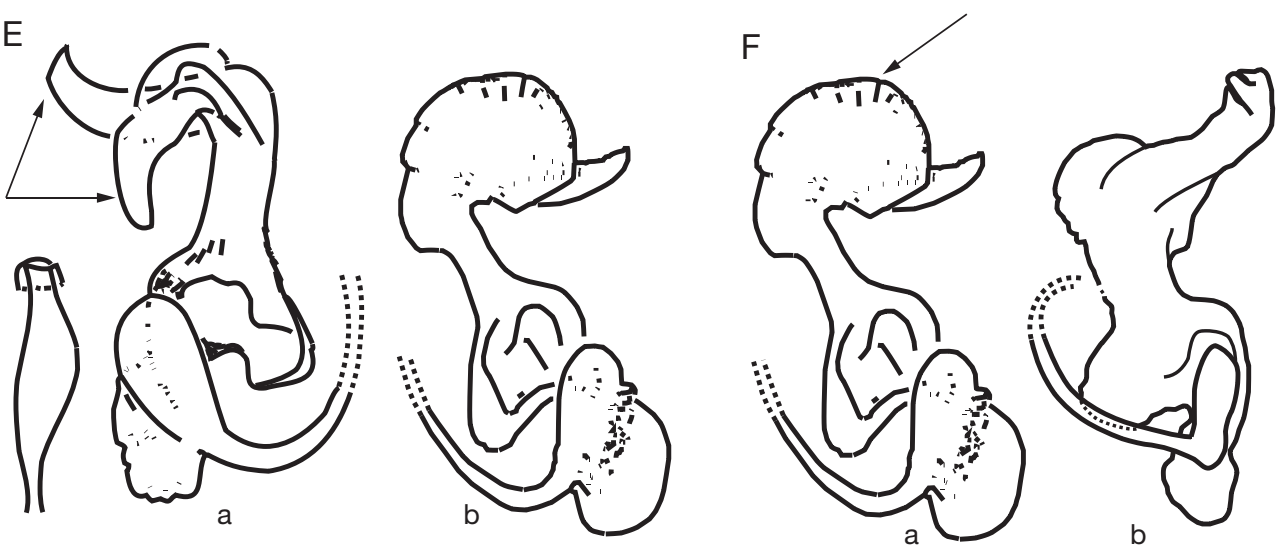

FIG. 8. - Illustration of identification key for Cichlidogyrus Paperna, 1960 species: A, presence (a) or absence (b) of an auxiliary plate associated with the male apparatus; $\mathbf{B}$, presence of two (a) or one (b) auxiliary plate associated with the male apparatus; C, size of the auxiliary plate associated with the male apparatus (small [a], large [b]); $\mathbf{D}$, shape of the accessory piece (S-shaped [a], bent at right angle [b]); $\mathbf{E}$, shape of the extremity of the accessory piece (presence [a] or absence [b] of large perpendicular diverticles); $\mathbf{F}$, shape of the accessory piece convex face (presence [a] or absence [b] of a large semi-circular extension). 
A
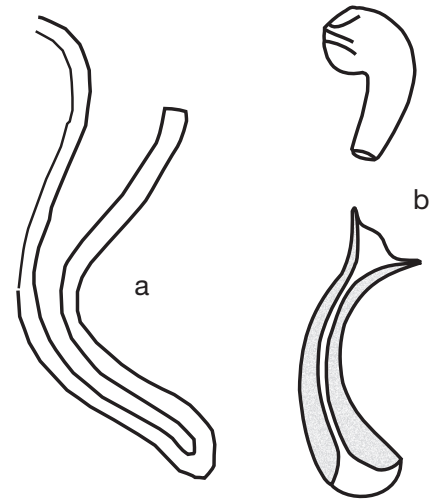

C

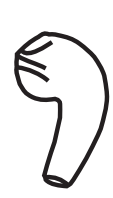

a

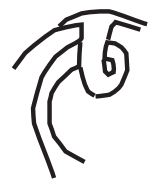

b

$\mathrm{E}$
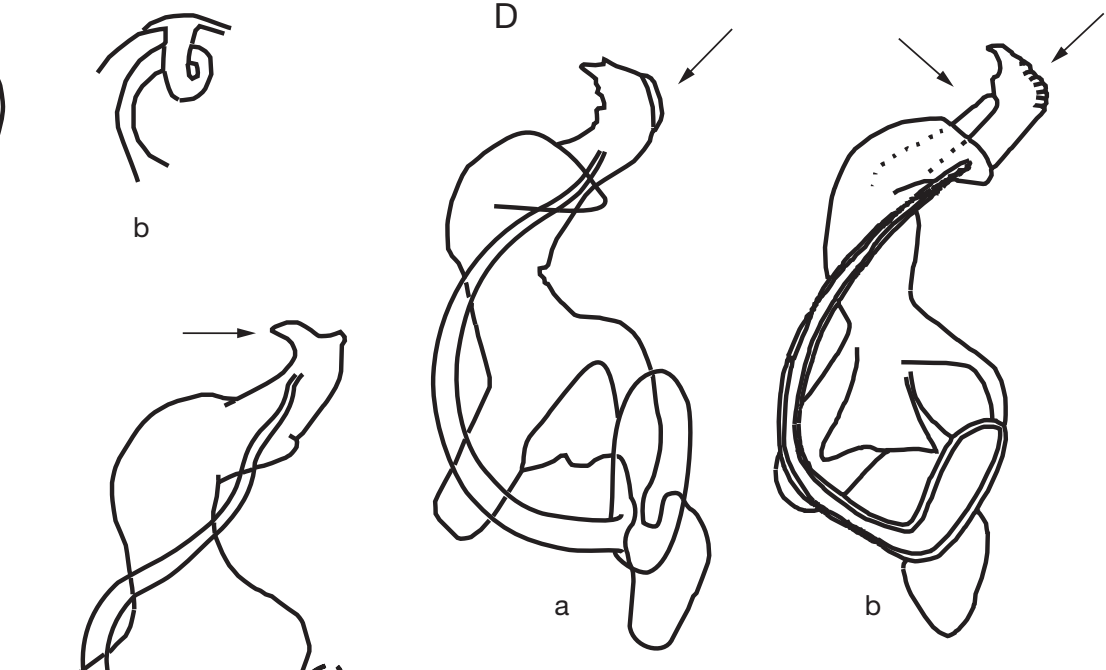

$\mathrm{F}$

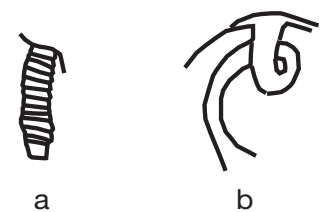

FIG. 9. - Illustration of identification key for Cichlidogyrus Paperna, 1960 species: A, shape of the sclerotised vagina (long and narrow [a], large and/or short [b]); B, shape of the accessory piece (T-shaped [a], ending in a hook [b]); C, shape of the vagina (short conical and right angle folded [a], tubular [b]); D, shape of the accessory piece ending convex face (smooth [a], crenulated [b]); E, shape of the accessory piece ending (folded back [a], hook shaped [b]); F, shape of vagina wall (annulated [a], smooth [b]). 


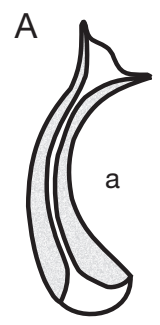

B

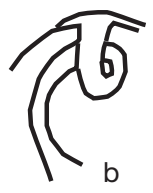

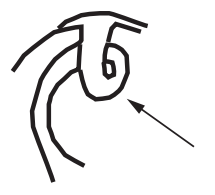

a
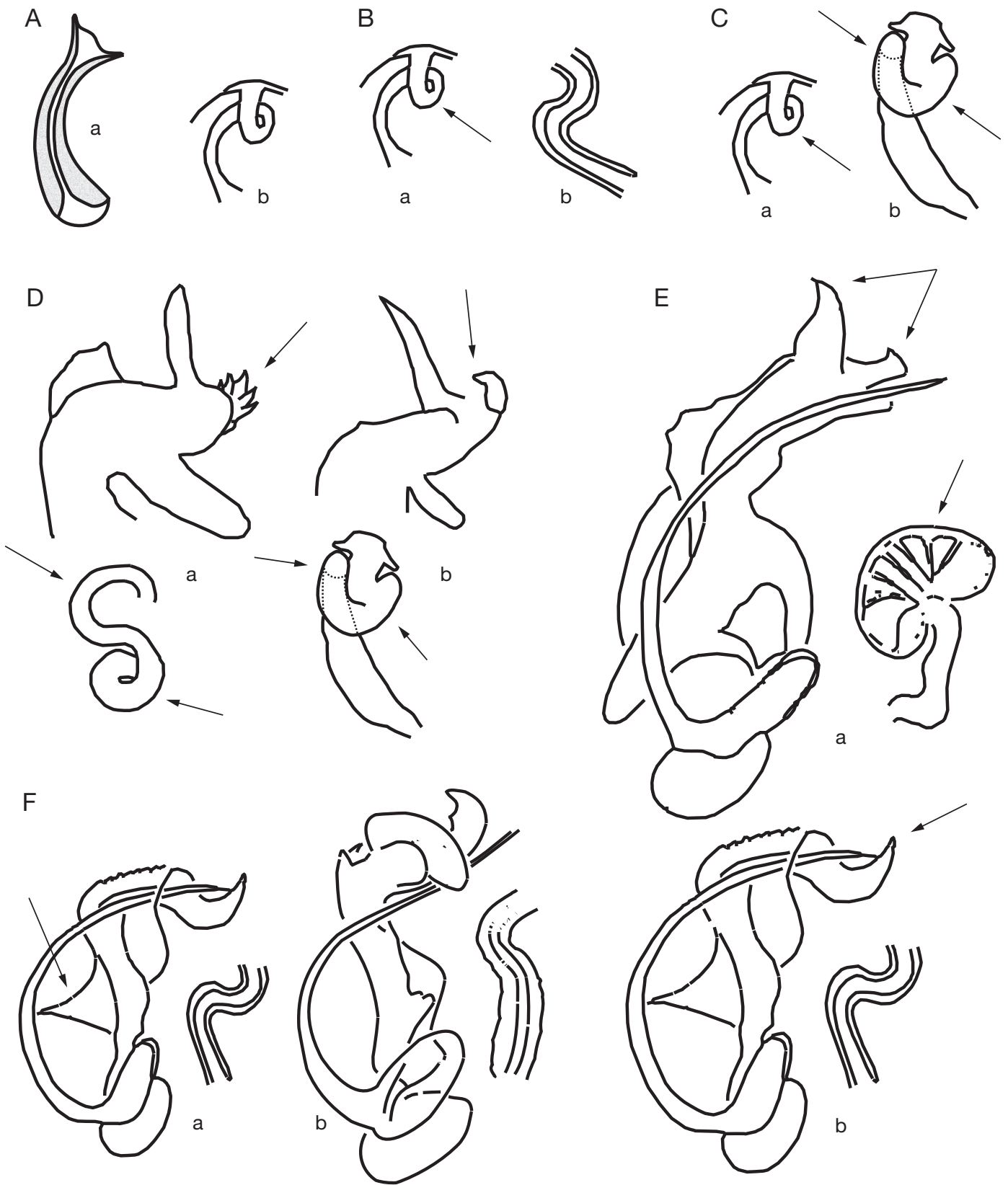

FIG. 10. - Illustration of identification key for Cichlidogyrus Paperna, 1960 species: A, shape of the vagina (straight or slightly curved [a], well curved or looped [b]); B, shape of the vagina (looped [a], curved [b]); C, shape of vagina (one [a] or two semi [b] loops); $\mathbf{D}$, shape of vagina and accessory piece extremity (semi-loops in the same plane and crenulated [a], semi-loops in perpendicular planes and single-hooked [b]); $\mathbf{E}$, shape of accessory piece extremity and vagina (ending in two points and characteristic enlargement [a], ending in a single point and no enlargement [b]); $\mathbf{F}$, presence (a) or absence (b) of a triangular extension at proximal third of the accessory piece. 
62. Accessory piece forming a large gutter-like portion at its base (Fig. 12A, a)

- Narrow accessory piece (Fig. 12A, b)

63. Accessory piece with a rounded bulge at distal third (Fig. 12B, a)

Cichlidogyrus dionchus

- Accessory piece without a rounded bulge at distal third (Fig. 12B, b) . 64

64. Accessory piece with double ending (Fig. 12C, a)

- Accessory piece with single ending (Fig. 12C, b)

Cichlidogyrus digitatus

65. Accessory piece ending in a thin and long hook (Fig. 12D, a) .... Cichlidogyrus berradae

- Accessory piece ending in a wide and short hook (Fig. 12D, b) 66

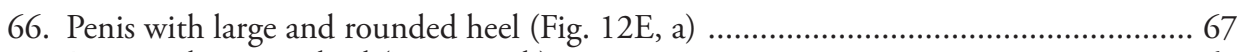

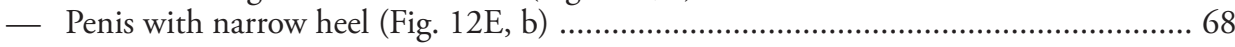

67. Penis extremity folded back, accessory piece closed C-shaped (Fig. 12F, a)

- Penis and accessory piece open C-shaped (Fig. 12F, b)

Cichlidogyrus reversati

68. Accessory piece $>27 \mu \mathrm{m}$ (Fig. 12G, a) Cichlidogyrus halinus

- Accessory piece $<26 \mu \mathrm{m}$ (Fig. 12G, b)

Cichlidogyrus yanni Cichlidogyrus quaestio

69. Spirally coiled penis, length more than $350 \mu \mathrm{m}$ (Fig. 13A, a) .................................. 70

— Not spirally coiled penis, length less than $150 \mu \mathrm{m}$ (Fig. 13A, b) .............................. 72

70. Penis with 4 or 5 turns, not spirally coiled vagina (Fig. 13B, a) ...... Cichlidogyrus euzeti

— Penis with more than 4 or 5 turns, spirally coiled vagina (Fig. 13B, b) ..................... 71

71. Penis with 8 or 9 turns, length about $450 \mu \mathrm{m}$ (Fig. 13C, a) ..... Cichlidogyrus longicirrus

- Penis with 14 or 15 turns, length about $1500 \mu \mathrm{m}$ (Fig. 13C, b) ... Cichlidogyrus sanseoi

72. Penis with large diameter, slightly sinuous, without heel (Fig. 13D, a)

- Filiform penis (Fig. 13D, b) Cichlidogyrus arfii

73. Nearly straight penis (Fig. 13E, a) Cichlidogyrus albareti

- Curved penis (Fig. 13E, b) 74

74. G-shaped penis (Fig. 13F, a) Cichlidogyrus teugelsi

- Single-looped penis (Fig. 13F, b) . 75

75. Long vagina, accessory piece round ended (Fig. 14A, a) ........... Cichlidogyrus nandidae

— Short vagina, accessory piece ending in a hook (Fig. 14A, b) ... Cichlidogyrus bychowskii

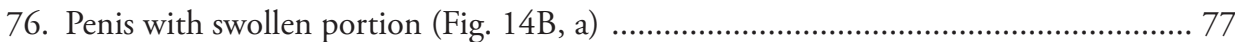

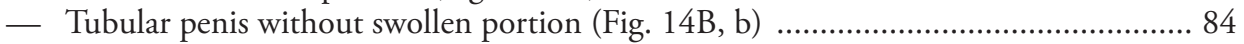

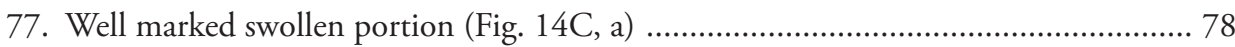

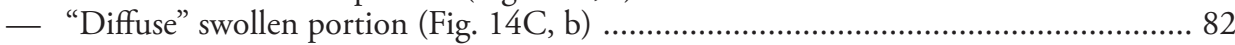

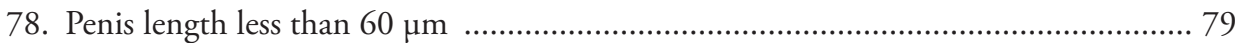

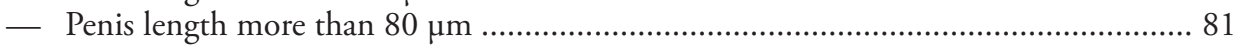


A

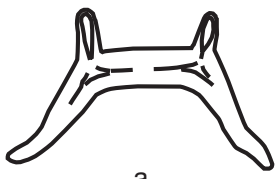

a
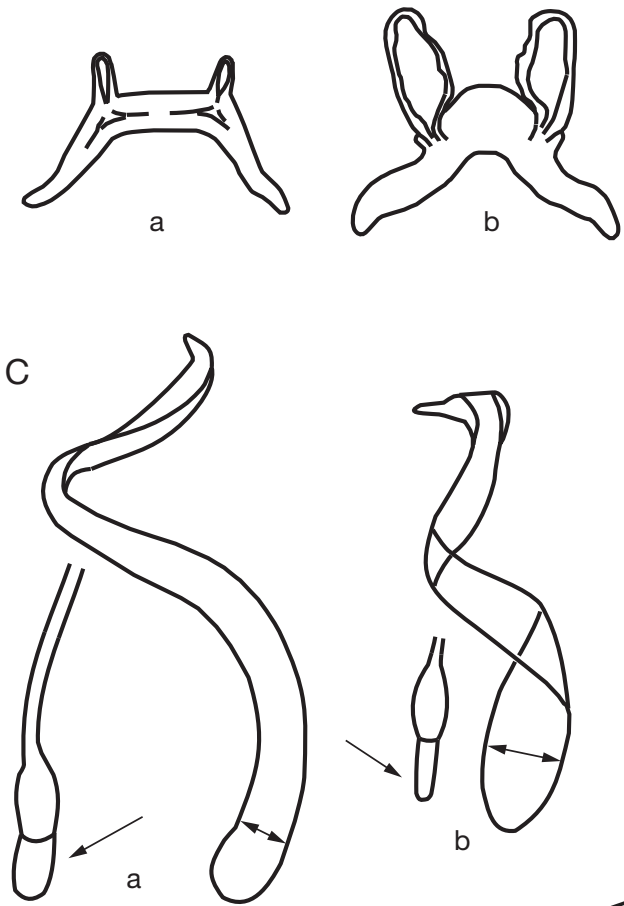

E

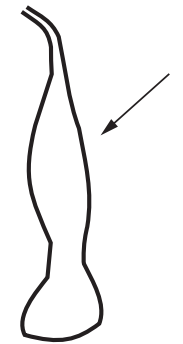

a

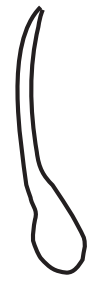

b

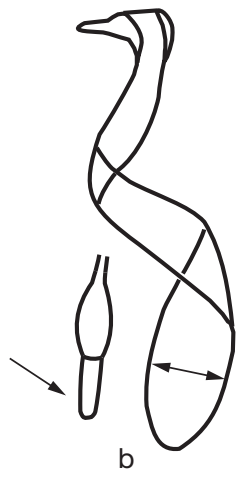

B

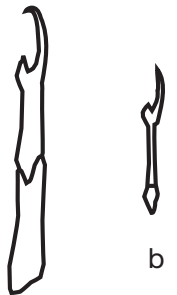

a

D
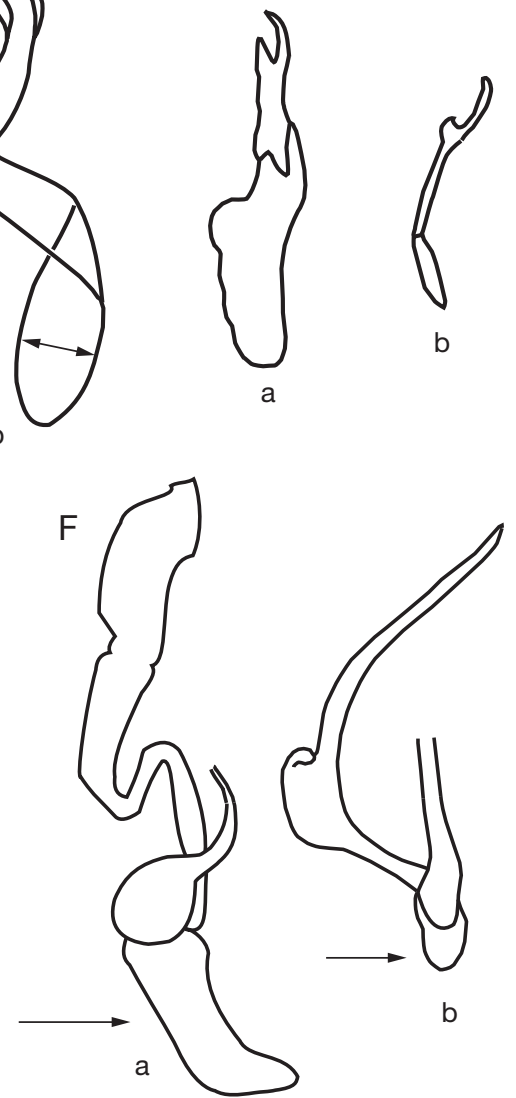

FIG. 11. - Illustration of identification key for Cichlidogyrus Paperna, 1960 species; A, size of auricle on the dorsal bar (reduced [a], developed [b]); B, relative size of uncinulus pair I (large [a], small [b]); C, shape of the accessory piece base and penis heel (narrow and large [a], wide and thin [b]); D, relative size of uncinulus pair I (large [a], small [b]); E, presence (a) or absence (b) of a swollen portion in the penis; $\mathbf{F}$, size of the penis heel and of the accessory piece (well developed and S-shaped [a], poorly developed and c-shaped [b]). 


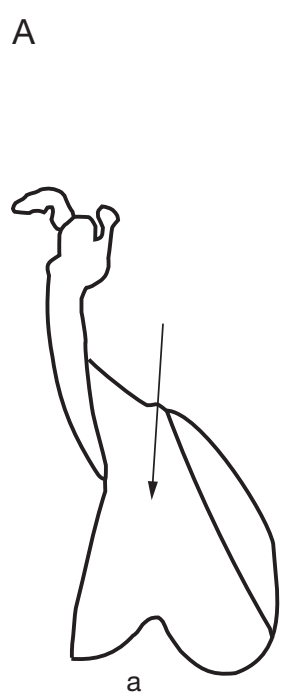

C
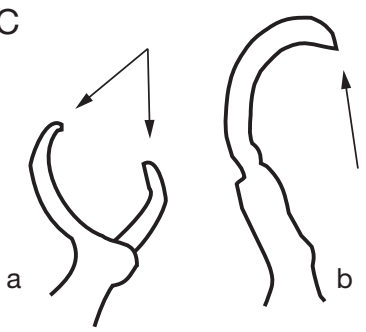

$\mathrm{D}$
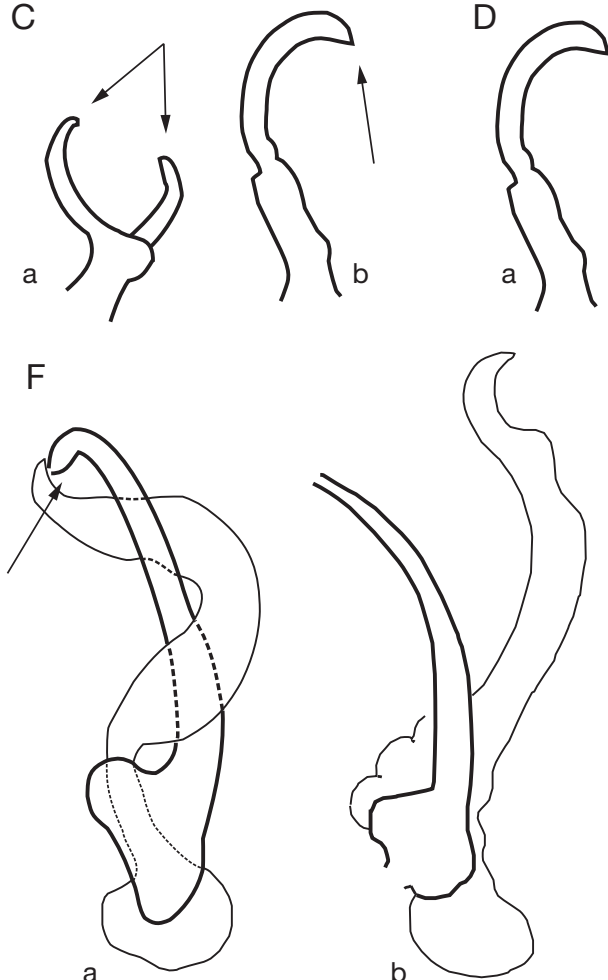

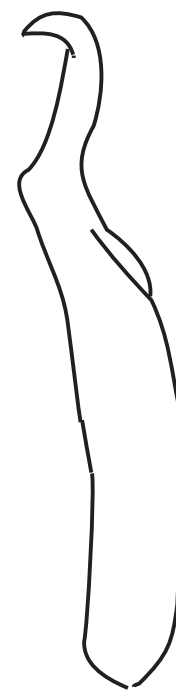

b
B

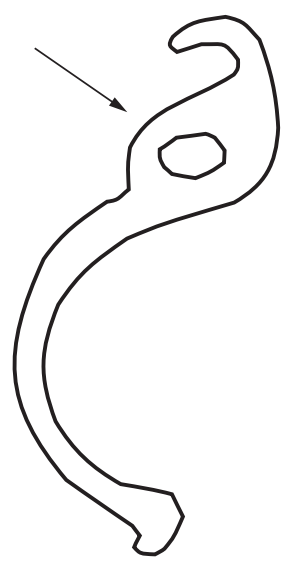

a

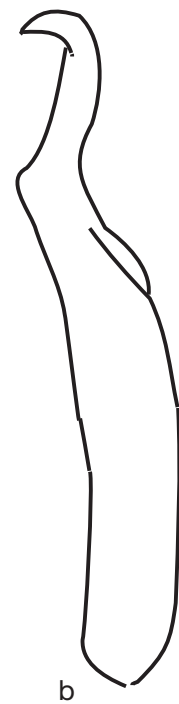

b
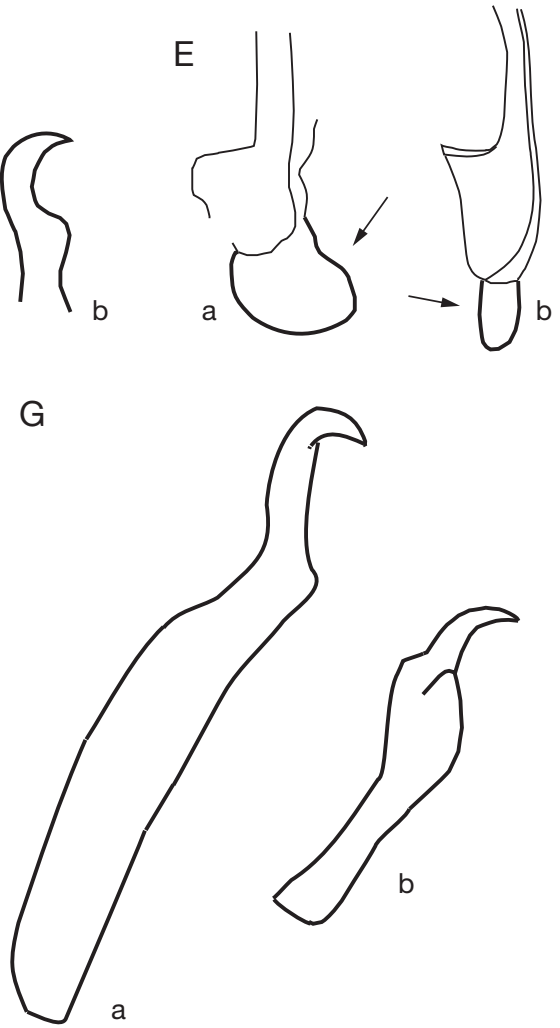

FIG. 12. - Illustration of identification key for Cichlidogyrus Paperna, 1960 species: A, shape of the accessory piece base (gutter-like [a], narrow [b]); B, presence (a) or absence (b) of a round bulge at distal third of the accessory piece; $\mathbf{C}$, shape of the accessory piece ending (double [a], single [b]); $\mathbf{D}$, shape of the accessory piece ending hook (thin and long [a], wide and short [b]); $\mathbf{E}$, shape of penis heel (wide [a], narrow [b]); F, shape of penis extremity and accessory piece (folded back and close C [a], straight and open C [b]); $\mathbf{G}$, size of accessory piece (long [a], short [b]). 
A
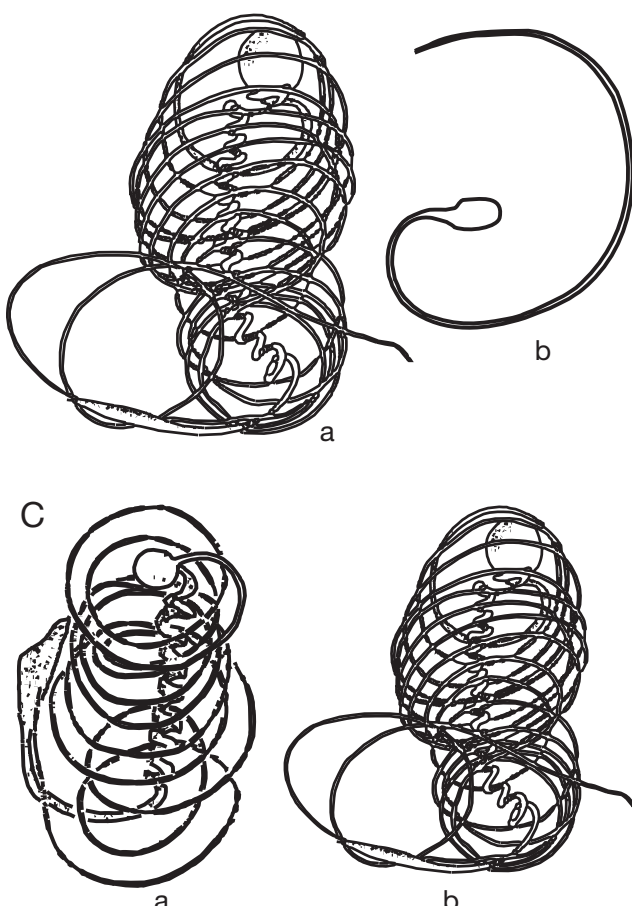

a

$\mathrm{E}$

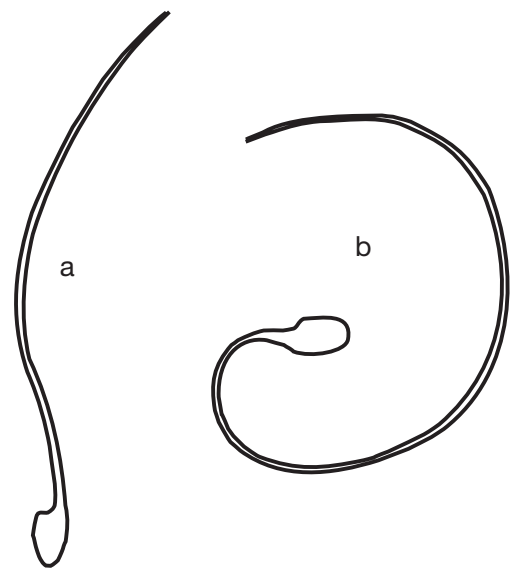

B
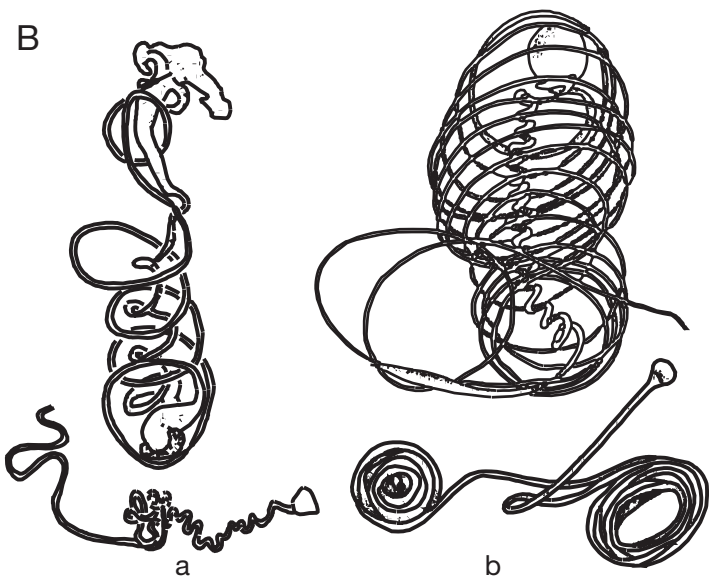

D
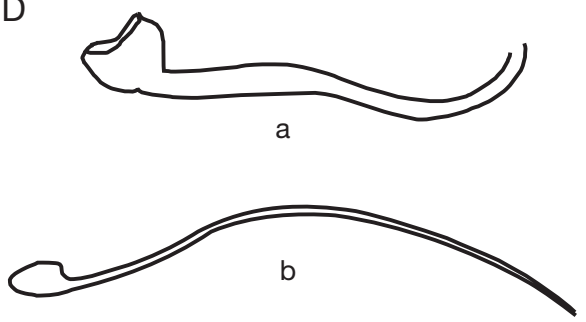

$\mathrm{F}$
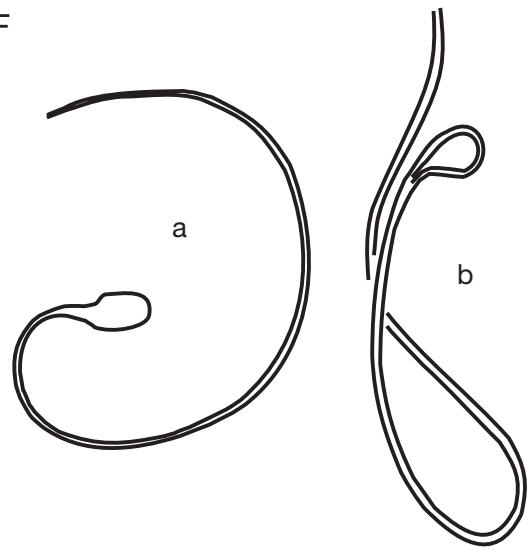

FIG. 13. - Illustration of identification key for Cichlidogyrus Paperna, 1960 species: A, shape of the penis (spirally coiled [a], not spirally coiled [b]); B, number of turns in spirally coiled penis and shape of the sclerotised vagina (4-5 and sinuous [a], more than 4-5 and spirally coiled [b]); C, number of turns in spirally coiled penis (8-9 [a], 14-15 [b]); D, penis diameter (large [a], filiform [b]); E, shape of the penis (straight [a], curved $[\mathbf{b}])$; $\mathbf{F}$, shape of the penis ( $[\mathbf{a}]$, single looped $[\mathbf{b}]$ ). 
79. No visible vagina, developed swollen portion (more than half penis total length) (Fig. 14D, a) .

- Visible S-shaped vagina, reduced swollen portion (less than one fifth of penis total length) (Fig. 14D, b)

80. Accessory piece ending in a hook (Fig. 14E, a)

Cichlidogyrus amphoratus

- Accessory piece bifurcated, lined along convex face with tubercles (Fig. 14E, b)

Cichlidogyrus ornatus

81. Accessory piece with heavily sclerotised portion L-shaped, uncinuli pairs II and III to VII $(11$ and $13 \mu \mathrm{m})($ Fig. 14F, a)

Cichlidogyrus giostrai

- Accessory piece with heavily sclerotised portion C-shaped, uncinuli pairs II and III to VII (16 and $20 \mu \mathrm{m})$ (Fig. 14F, b)

Cichlidogyrus njinei

82. Penis length less than $40 \mu \mathrm{m}$

- Penis length more than $55 \mu \mathrm{m}$

Cichlidogyrus lagoonaris

83. Short, large and conical vagina (Fig. 14G, a)

83

- Short, thin and S-shaped vagina (Fig. 14G, b)

Cichlidogyrus zambezensis

84. Penis length less than $60 \mu \mathrm{m}$ Cichlidogyrus karibae

- Penis length more than $60 \mu \mathrm{m}$ 85

85. Sclerotised vagina 92

- Vagina not visible 86

86. Single C-shaped accessory piece with a thin extension at its base (Fig. 15A, a) Cichlidogyrus levequei

- Complex accessory piece with well-sclerotised C-shaped portion (Fig. 15A, b) . 87

87. Accessory piece with a C-shaped portion sharply arched, with thin plates at each extremity (Fig. 15B, a) Cichlidogyrus slembroucki

- Accessory piece with C-shaped portion slightly arched, without plates at its extremities, and a digitations at its middle (Fig. 15B, b) Cichlidogyrus acerbus

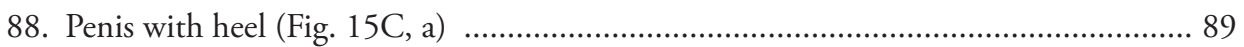

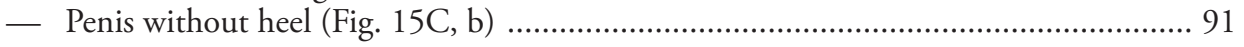

89. Very thin heel, accessory piece ending in claws (Fig. 15D, a) ...... Cichlidogyrus fontanai

- Developed heel, accessory piece with forked ending (Fig. 15D, b) ........................... 90

90. Penis length less than $40 \mu \mathrm{m}$, large dorsal bar auricles (Fig. 15E, a)

- Penis length more than $40 \mu \mathrm{m}$, dorsal bar auricles smaller (Fig. 15E, b)

Cichlidogyrus rognoni Cichlidogyrus bifurcatus

91. Penis length more than $40 \mu \mathrm{m}$, accessory piece thin at level of insertion on basal bulb (Fig. 15F, a) Cichlidogyrus haplochromii

- Penis length less than $37 \mu \mathrm{m}$, accessory piece large at level of insertion (Fig. 15F, b) ...... Cichlidogyrus tilapiae

92. Accessory piece straight or slightly curved (Fig. 16A, a) .......................................... 93

— Accessory piece sharply curved (Fig. 16A, b) ........................................................ 95 


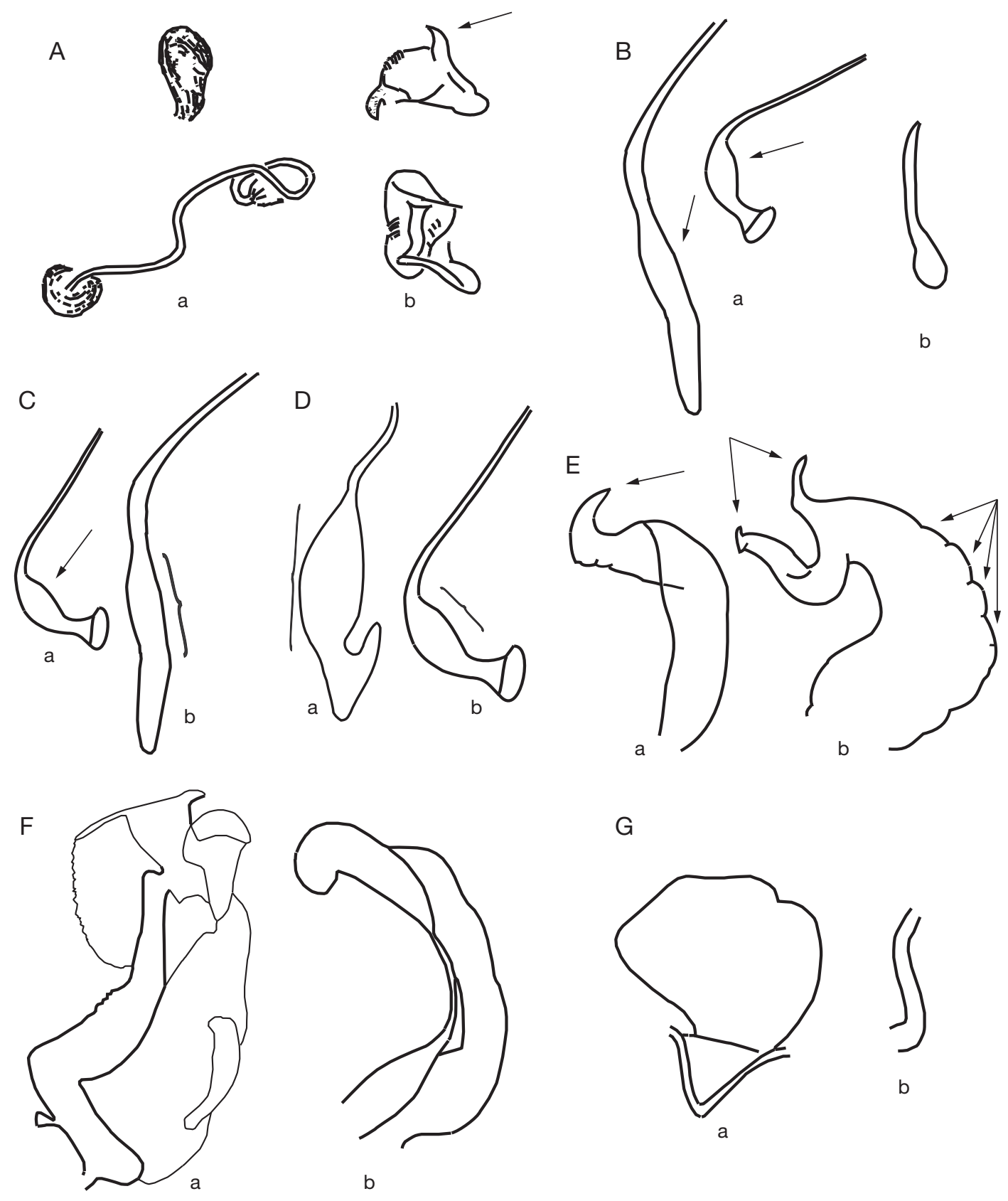

FIG. 14. - Illustration of identification key for Cichlidogyrus Paperna, 1960 species: A, size of the sclerotised vagina and shape of accessory piece ending (long and rounded [a], short and hooked [b]); B, presence (a) or absence (b) of a swollen portion in the penis; $\mathbf{C}$, well marked (a) or diffuse (b) swollen portion in the penis; $\mathbf{D}$, relative size of the swollen portion in the penis (developed [a], reduced [b]); $\mathbf{E}$, shape of accessory piece ending (hooked [a], bifurcated [b]) and presence of tubercles along the convex face; $\mathbf{F}$, shape of accessory piece heavily sclerotised portion ( $\mathrm{L}[\mathbf{a}], \mathrm{C}[\mathbf{b}])$; G, shape of the sclerotised vagina (large and conical $[\mathbf{a}]$, thin and S-shaped [b]). 


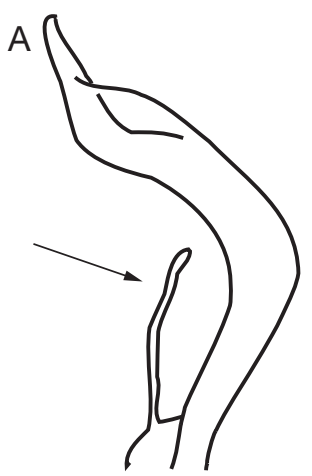

a

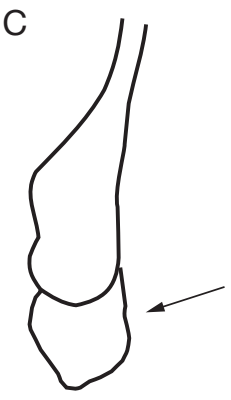

a

E
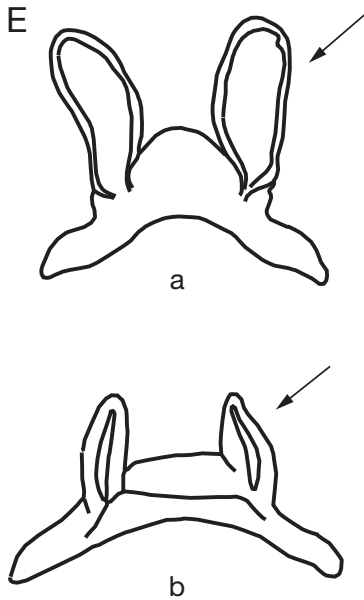

b
$\mathrm{B}$
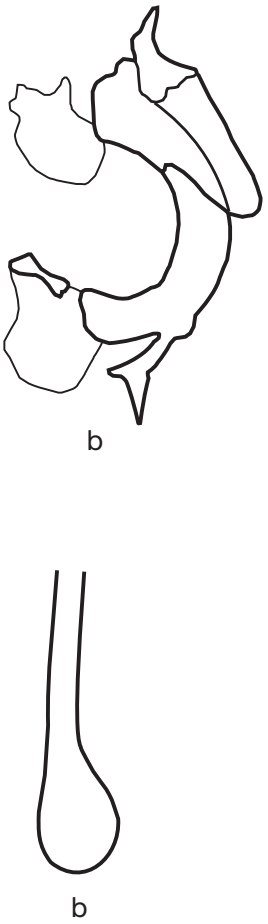

a

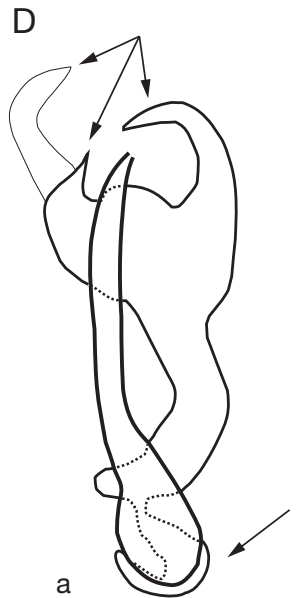

F

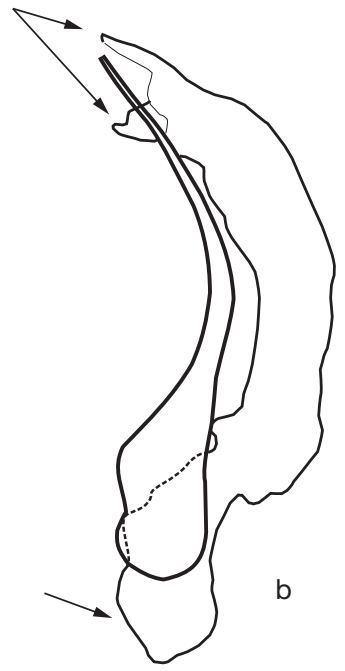

a

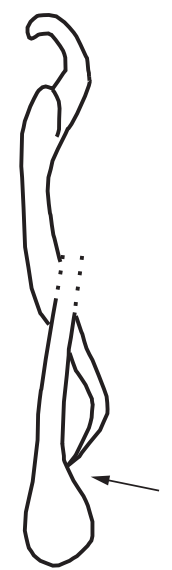

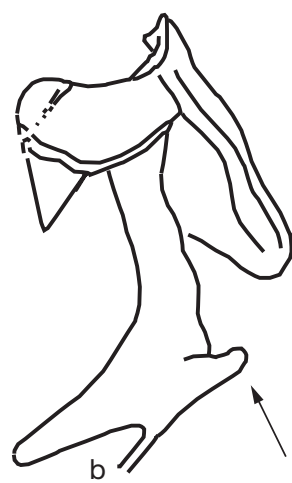

a

$\mathrm{b}$

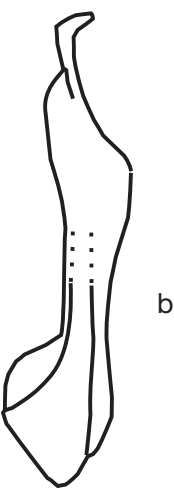

FIG. 15. - Illustration of identification key for Cichlidogyrus Paperna, 1960 species: A, shape of the accessory piece (simple with extension [a], complex [b]); B, presence (a) or absence (b) of thin plates at the extremity of the accessory piece; C, presence (a) or absence (b) of a penis heel; $\mathbf{D}$, size of the penis heel and shape of the accessory piece ending (thin and presence of claws [a], developed and forked [b]); E, size dorsal bar auricles (large [a], small [b]); F, size of the penis and width of accessory piece insertion at the level of the penis (more than $40 \mu \mathrm{m}$ and thin [a], less than $37 \mu \mathrm{m}$ and large [b]). 

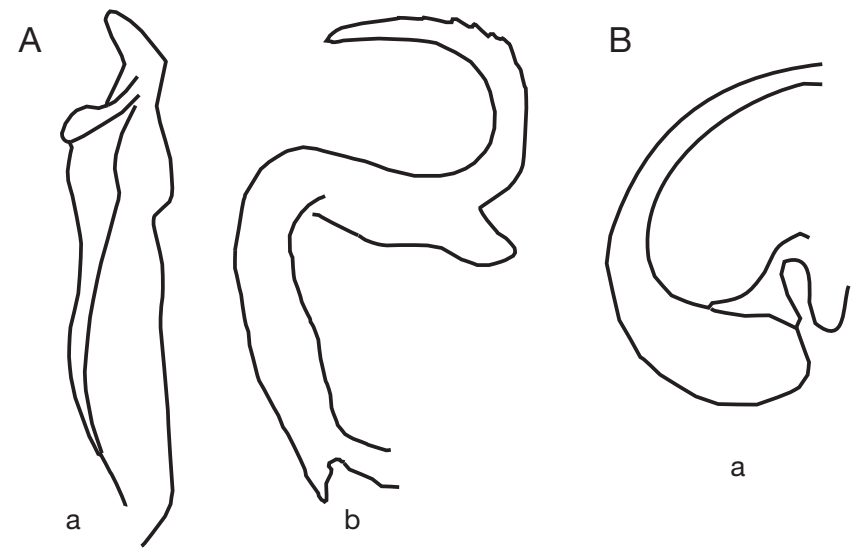

a

C

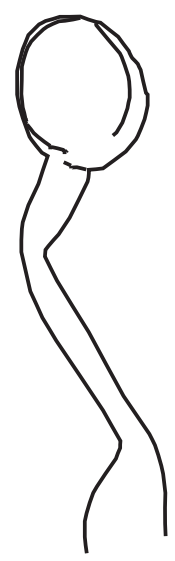

a

D

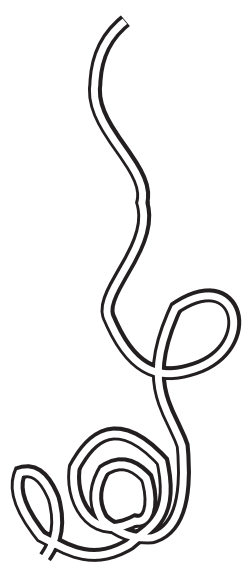

b

$\mathrm{E}$

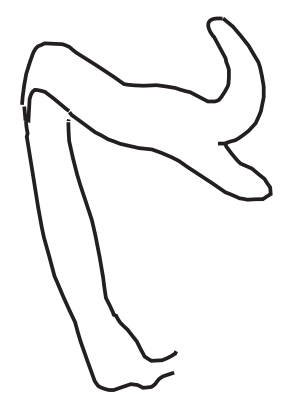

a

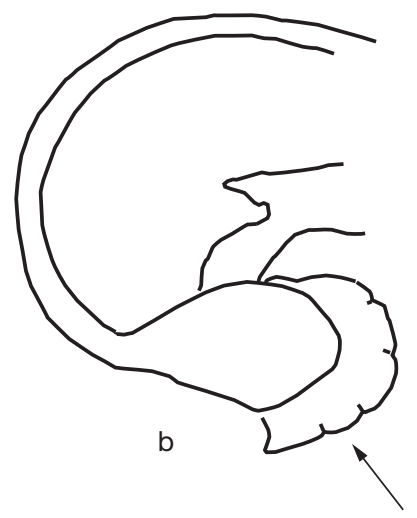

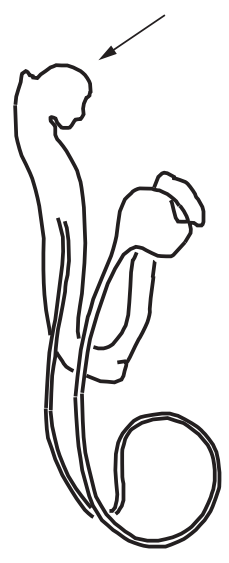

a

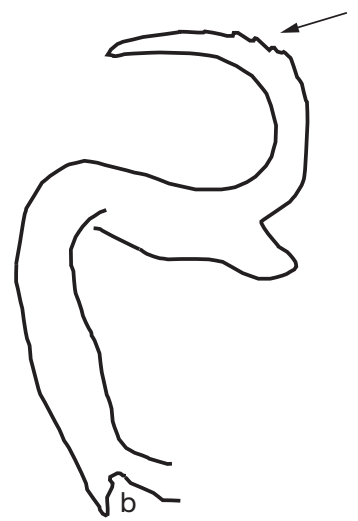

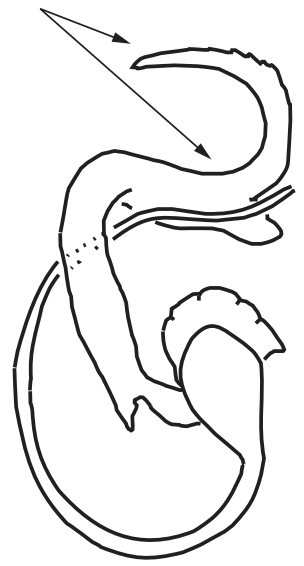

b

FIG. 16. - Illustration of identification key for Cichlidogyrus Paperna, 1960 species: A, shape of accessory piece (straight [a], sharply curved [b]); B, absence (a) or presence (b) of a penis heel; C, shape of the sclerotised vagina (straight and large [a], sinuous and thin $[\mathbf{b}])$; $\mathbf{D}$, shape of the accessory piece ending and of the penis (rounded and looped [a], forked and C [b]); E, accessory piece extremities shape and length (smooth and equal [a], rough and unequal [b]). 
93. Penis with no visible heel (Fig. 16B, a) Cichlidogyrus longipenis

— Penis with developed heel (Fig. 16B, b) 94

94. Straight and large vagina, penis length $70 \mu \mathrm{m}$ (Fig. 16C, a) ......... Cichlidogyrus sclerosus

— Long, thin and sinuous vagina, penis length $145 \mu \mathrm{m}$ (Fig. 16C, b) ... Cichlidogyrus cirratus

95. Accessory piece with rounded ending, looped penis (Fig. 16D, a) ... Cichlidogyrus amieti

- Accessory piece forked ending, C-shaped penis (Fig. 16D, b) 96

96. Extremities of accessory piece smooth and about equal in length (Fig. 16E, a) Cichlidogyrus cubitus

- Extremities of accessory piece highly unequal in length, the longer one with rough patch on its convex face (Fig. 16E, b) Cichlidogyrus louipaysani

\section{REFERENCES}

APPLEBy C. 1996. - Variability of the opisthaptoral hard parts of Gyrodactylus callariatis Malmberg, 1957 (Monogenea: Gyrodactylidae) from Atlantic cod Gadus morhua L. in the Oslo Fjord, Norway. Systematic Parasitology 33: 199-207.

Bender H.-G. 1979. - Zur morphologie und biologie von Enterogyrus hemihaplochromii n. sp. (Trematodes, Monogenea, Dactylogyridae, Ancyrocephalinae), einem endoparasiten bei Hemihaplochromis multicolor (Teleostei, Cichlidae). Ph.D. thesis, Zulassungsarbeit, Zoologisches Institut der Universität Hohenheim, Hohenheim, Germany.

BiLONG BiLONG C. F. 1988. - Enterogyrus malmbergi n. sp. (Monogenea-Ancyrocephalidae) parasite de l'estomac du Cichlidae Tilapia nilotica Linné, 1757 au Sud-Cameroun. Annales de la Faculté des Sciences de Yaoundé, Biologie-Biochimie 5: 51-58.

Bilong Bilong C. F., Birgi E. \& Lambert A. 1989. Enterogyrus melenensis n. sp. (Monogenea, Ancyrocephalidae), parasite stomacal de Hemichromis fasciatus Peters, 1857 (Téléostéen, Cichlidae) du Sud-Cameroun. Revue de Zoologie africaine - Journal of African Zoology 103: 99-105.

Bilong Bilong C. F. \& Euzet L. 1995. — Onchobdella bopeleti n. sp. (Monogenea, Ancyrocephalidae) parasite branchial de Hemichromis fasciatus (Peters, 1857) (Cichlidae). Journal of African Zoology 109: 253-258.

Bilong Bilong C. F., EuZET L. \& Birgi E. 1996. Monogenean stomach parasites of cichlid fishes from Cameroon: two new species of the genus Enterogyrus Paperna, 1963 (Ancyrocephalidae). Systematic Parasitology 34: 37-42.

Bilong Bilong C. F., Birgi E. \& Euzet L. 1991. Enterogyrus barombiensis n. sp. (Monogenea, Ancyrocephalidae) parasite stomacal de trois Cichlidae endémiques du lac de cratère Barombi Mbo (Cameroun). Annales de Parasitologie humaine et comparée 66: 105-108.

Bilong Bilong C. F., Birgi E. \& Euzet L. 1994. —
Urogyrus cichlidarum gen.nov., sp.nov., Urogyridae fam.nov., monogène parasite de la vessie urinaire de poissons cichlidés au Cameroun. Canadian Journal of Zoology 72: 561-566.

Birgi E. \& EUZET L. 1983. - Monogènes parasites des poissons des eaux douces du Cameroun. Présence des genre Cichlidogyrus et Dactylogyrus chez Aphyosemion (Cyprinodontidae). Bulletin de la Société zoologique de France 108: 101-106.

Birgi E. \& Lambert A. 1986. - Présence chez un Nandidae (Téléostéen), Polycentropsis abbreviata Boulenger, 1901, du genre Cichlidogyrus (Monogenea, Monopisthocotylea, Ancyrocephalidae). Description de Cichlidogyrus nandidae n. sp. Annales de Parasitologie humaine et comparée 61: 521-528.

Birgi E. \& LAmberT A. 1987. — À propos de Cichlidogyrus euzeti Birgi \& Lambert, 1986 décrit au Cameroun chez un Nandidae Polycentropsis abbreviata Boulenger, 1901. Annales de Parasitologie humaine et comparée 62: 103.

Caltran H., Silan P. \& Roux M. 1995. — Ligophorus imitans (Monogenea) ectoparasite de Liza ramada (Teleostei) I. Populations naturelles et variabilité morphologique. Écologie 26: 95-104.

Cone D. K., Gratzek J. B. \& Hoffman G. L. 1987. — A study of Enterogyrus sp. (Monogenea) parasiting the foregut of captive Pomacanthus paru (Pomacanthidae) in Georgia. Canadian Journal of Zoology 65: 312-316.

Dossou C. 1973. - Recherches sur les Monogènes parasites des poissons d'eau douce du Sud Dahomey. Ph.D. Thesis, Université des Sciences et Techniques du Languedoc, Montpellier, France et Université du Dahomey, Cotonou, Bénin, 217 p.

Dossou C. 1982. — Parasites de poissons d'eau douce du Bénin III. Espèces nouvelles du genre Cichlidogyrus (Monogenea) parasites de Cichlidae. Bulletin de l'I.F.A.N. 44: 295-322.

Dossou C. 1985. - Monogènes parasites de poissons d'eau douce au Bénin (Ouest-africain). National Ph.D. Thesis, Université Montpellier II, Montpellier, France, $116 \mathrm{p}$. 
Dossou C. \& Birgi E. 1984. — Monogènes parasites d'Hemichromis fasciatus Peters, 1857 (Teleostei, Cichlidae). Annales des Sciences naturelles, Zoologie 6: 101-109.

DouËLlOU L. 1993. - Monogeneans of the genus Cichlidogyrus Paperna, 1960 (Dactylogyridae: Ancyrocephalinae) from cichlid fishes of Lake Kariba (Zimbabwe) with descriptions of five new species. Systematic Parasitology 25: 159-186.

Eid N. \& Negm M. 1987. - Some morphological study on a new species of endoparasitic monogenetic Trematode Enterogyrus niloticus in the intestine of Tilapia nilotica. Journal of the Egyptian Veterinary Medical Association 47: 79-86.

ERGENS R. 1981. - Nine species of the genus Cichlidogyrus Paperna, 1960 (Monogenea: Ancyrocephalinae) from Egyptian fishes. Folia Parasitologica 28: 205-214.

ERGENS R. \& GELNAR M. 1985. - Experimental verification of the effect of temperature on the size of hard parts of opistohaptor of Gyrodactylus katharineri Malmberg, 1964. Folia Parasitologica 32: 377-380.

Froese R. \& Pauly D. (eds) 2009. — FishBase. World Wide Web electronic publication. http://www.fishbase. org (September 2009).

Gussev A. V. \& Fernando C. H. 1973. - Dactylogyridae (Monogenoidea) from stomach of Fishes. Folia Parasitologica (Praha) 20: 207-212.

KHIDR A. A. 1990. - Population dynamics of Enterogyrus cichlidarum (Monogenea: Ancyrocephalinae) from the stomach of Tilapia spp. in Egypt. International Journal for Parasitology 20: 741-747.

Lim L. H. S., TimOFEEVA T. A. \& GibSON D. I. 2001. Dactylogyridean monogeneans of the siluriform fishes of the Old World. Systematic Parasitology 50: 159-197.

MARKEVICH A. P. 1934. - [Parasitic diseases of fish and their control]. Publ. Koiz., Leningrad, 100 p. (in Russian).

N'Douba V., Thys Van Den Audenaerde D.F.E. \& PARISElle A. 1997. - Description d'une espèce nouvelle de Monogène ectoparasite branchial de Tilapia guineensis (Bleeker, 1862) (Cichlidae) en Côte d'Ivoire. Journal of African Zoology 111: 429-433.

PAPERNA I. 1960. - Studies on Monogenetic Trematodes in Israel. 2 Monogenetic Trematodes of Cichlids. Bamidgeh, Bulletin of Fish Culture in Israel 12: 2033.

PAPERNA I. 1963. - Enterogyrus cichlidarum n. gen. n. sp., a monogenetic Trematode parasitic in the intestine of Fish. The Bulletin of the Research Council of Israel 11B: 183-187.

PAPERNA I. 1964. - Parasitic helminths of inland-water fishes in Israel. Israel Journal of Zoology 13: 1-26.

PAPERNA I. 1965. - Monogenetic Trematodes collected from fresh water fish in southern Ghana. Bamidgeh, Bulletin of Fish Culture in Israel 17: 107-115.
PAPERNA I. 1968. - Monogenetic Trematodes collected from fresh water fish in Ghana. Second report. Bamidgeh, Bulletin of Fish Culture in Israel 20: 80100.

Paperna I. 1969. - Monogenetic Trematodes of the fish of the Volta basin and South Ghana. Bulletin de l'I.F.A.N. 31: 840-880.

PAPERNA I. 1973. - New species of Monogenea (Vermes) from African freshwater fish. A preliminary report. Revue de Zoologie et de Botanique africaines 87: 505518.

PAPERNA I. 1979. - Monogenea of inland water fish in Africa. Annales du Musée royal d'Afrique centrale, sér in- $8^{\circ}$ (Zool.) 226: 1-131.

Paperna I. \& Thurston J. P. 1969. - Monogenetic Trematodes collected from cichlid fish in Uganda; including the description of five new species of Cichlidogyrus. Revue de Zoologie et de Botanique africaines 79: 15-33

Pariselle A. 1995. - Études des parasites de Cichlidae en Afrique de l'Ouest, in AGNĖSE J.-F. (ed.), Comptes rendus de l'atelier biodiversité et aquaculture. CRO Abidjan: 44-52.

PARISELle A. 1996. - Diversité, spéciation et évolution des Monogènes branchiaux de Cichlidae en Afrique de l'Ouest. PhD Thesis, Université de Montpellier II, Montpellier, France, 199 p.

Pariselle A., Bilong Bilong C. F. \& EuZet L. 2003. Four new species of Cichlidogyrus Paperna, 1960 (Monogenea, Ancyrocephalidae) all gill parasites from African mouthbreeder tilapias of the genera Sarotherodon and Oreochromis (Pisces, Cichlidae), with a re-description of $C$. thurstonae Ergens, 1981. Systematic Parasitology 56: 201-210.

Pariselle A. \& Euzet L. 1994. - Three new species of Cichlidogyrus Paperna, 1960 (Monogenea, Ancyrocephalidae) parasitic on Tylochromis jentinki (Steindachner, 1895) (Pisces, Cichlidae) in West Africa. Systematic Parasitology 29: 229-234.

Pariselle A. \& Euzet L. 1995a. — Trois Monogènes nouveaux parasites branchiaux de Pelmatochromis buettikoferi (Steindachner, 1895) (Cichlidae) en Guinée. Parasite 2: 203-209.

PARISElle A. \& EUZET L. 1995b. - Scutogyrus n. g. (Monogenea, Ancyrocephalidae) for Cichlidogyrus longicornis minus Dossou, 1982, C. longicornis and C. l. gravivaginus Paperna and Thurston, 1969, with descriptions of three new species parasitic on African Cichlids. Journal of the Helminthological Society of Washington 62: 157-173.

Pariselle A. \& EuZet L. 1995c. - Gill parasites of the genus Cichlidogyrus Paperna, 1960 (Monogenea, Ancyrocephalidae) from Tilapia guineensis (Bleeker, 1862), with descriptions of six new species. Systematic Parasitology 30: 187-198.

Pariselle A. \& Euzet L. 1996. — Cichlidogyrus Paperna, 
1960 (Monogenea, Ancyrocephalidae): gill parasites from West African Cichlidae of the subgenus Coptodon Regan, 1920 (Pisces), with descriptions of six new species. Systematic Parasitology 34: 109-124.

Pariselle A. \& EuZet L. 1997. - New species of Cichlidogyrus Paperna, 1960 (Monogenea, Ancyrocephalidae) from the gills of Sarotherodon occidentalis (Daget) (Osteichthyes, Cichlidae) in Guinea and Sierra Leone (West Africa). Systematic Parasitology 38: 221-230.

Pariselle A. \& EuZet L. 1998. - Five new species Cichlidogyrus Paperna, 1960 (Monogenea: Ancyrocephalidae) from Tilapia brevimanus, T. buttikoferi and T. cessiana from Guinea, Côte d'Ivoire and Sierra Leone (West Africa). Folia Parasitologica 45: 275-282.

Pariselle A. \& EuZet L. 2003. - Four new species of Cichlidogyrus (Monogenea: Ancyrocephalidae), gill parasites of Tilapia cabrae (Teleostei: Cichlidae) with discussion on relative length of haptoral sclerites. Folia Parasitologica 50: 195-201.

Pariselle A. \& Euzet L. 2004. - Two new species of Cichlidogyrus Paperna, 1960 (Monogenea, Ancyrocephalidae) gill parasites on Hemichromis fasciatus (Pisces, Cichlidae) in Africa, with remarks on geographical distribution. Parasite 11: 359-364.

Pariselle A., Lambert A. \& Euzet L. 1991. - A new type of haptor in mesoparasitic monogeneans of the genus Enterogyrus Paperna, 1963, with a description of Enterogyrus foratus n. sp. and E. coronatus n. sp., stomach parasites of Cichlids in West Africa. Systematic
Parasitology 20: 211-220.

Paugy D., LéVÊQue C. \& Teugels G. G. 2003. — Poissons d'eaux douces et saumâtres de l'Afrique de l'Ouest, Tome II. Faune et Flore Tropicales 40: 815 p.

Pouyaud L., Desmarais E., DeVeney M. \& Pariselle A. 2006. - Phylogenetic relationships among monogenean gill parasites (Dactylogyridae, Ancyrocephalinae) infecting tilapiine hosts (Cichlidae), systematic and evolutionary implications. Molecular Phylogenetics and Evolution 38: 241-249.

PRICE C. E. 1967. - The freshwater monogenetic trematodes of Africa. Revue de Zoologie et de Botanique Africaine 76: 375-391.

PRICE C. E. 1968. - A note on the monogenetic trematode genus Cichlidogyrus Paperna, 1960. Bamidgeh, Bulletin of Fish Culture in Israel 20: 100.

PRICE C. E. \& KirK R. G. 1967. — First description of a monogenetic trematode from Malawi. Revue de Zoologie et de Botanique africaines 76: 137-144.

Price C. E., Peebles H. E. \& Bamford T. 1969. - The Monogenean parasites of African fishes. IV. Two new species from South African hosts. Revue de Zoologie et de Botanique africaines LXXIX: 117-124.

RAKOTOFIRINGA S. \& EUZET L. 1983. - Monogènes parasites de Cichlidae (Teleostei) endémiques de Madagascar. Bulletin de la Société zoologique de France 108: 107-114.

ThurSTON J. P. 1970. - The incidence of Monogenea and parasitic Crustacea on the gills of fish in Uganda. Revue de Zoologie et de Botanique africaines 82: 111-129.

Submitted on 5 August 2008; accepted on 17 December 2008. 